\title{
ON NICHOLS ALGEBRAS OF DIAGONAL TYPE
}

\author{
IVÁN ANGIONO
}

\begin{abstract}
We give an explicit and essentially minimal list of defining relations of a Nichols algebra of diagonal type with finite root system. This list contains the wellknown quantum Serre relations but also many new variations. A conjecture by Andruskiewitsch and Schneider states that any finite-dimensional pointed Hopf algebra over an algebraically closed field of characteristic zero is generated as an algebra by its group-like and skew-primitive elements. As an application of our main result, we prove the conjecture when the group of group-like elements is abelian.
\end{abstract}

\section{INTRODUCTION}

1. Let $\mathbf{k}$ be an algebraically closed field of characteristic zero and let $\theta$ be a natural number. Let $\mathbf{q}=\left(q_{i j}\right)_{1 \leq i, j \leq \theta}$ be a matrix with invertible entries on $\mathbf{k}$ and let $V$ be a vector space of dimension $\theta$. The Nichols algebra associated to $\mathbf{q}$ is a graded connected Hopf algebra $\mathcal{B}(V)=\oplus_{n \geq 0} \mathcal{B}^{n}(V)$ with many favorable properties. It plays a fundamental role in the classification of finite-dimensional (or finite growth) pointed Hopf algebras. Precisely, a basic question in the classification Program [AS1] is the following:

Question 1. An, Question 5.9]: Given $(V, \mathbf{q})$, determine if the associated Nichols algebra $\mathcal{B}(V)$ is finite-dimensional. In such case, compute the dimension of $\mathcal{B}(V)$ and give a presentation by generators and relations.

The first part of this question has been answered by Heckenberger [H3], who obtained the list of all matrices $\mathbf{q}$ whose associated Nichols algebra has a finite root system. Roughly, this list contains three classes of matrices:

- Standard matrices $[\mathrm{AA}$ : they are associated with finite Weyl groups. Their root systems coincide with root systems of finite Cartan matrices. This family includes properly the so-called braidings of Cartan type, in particular the matrices related with the positive part of the small quantum groups.

- Matrices of super type [AAY, related with the positive part of quantized enveloping algebras of contragradient Lie superalgebras. Their root systems become from the corresponding Lie superalgebras.

- A finite list of exceptional matrices, whose associated diagram has connected components with at most 7 vertices, and the scalars defining these braidings are roots of unity of low order.

There are several answers to the second part of Question 1 under particular assumptions:

$\triangleright[\mathrm{L}]$ for the positive part of quantized enveloping algebras of semisimple Lie algebras and small quantum groups, using the full representation theory of quantum groups;

$\triangleright$ AS2 for braidings of Cartan type;

$\triangleright$ [A1] for braidings of standard type;

$\triangleright[\mathrm{Y}]$ for the positive part of quantized enveloping algebras of contragradient Lie superalgebras;

2010 Mathematics Subject Classification. 16W30.

The work was partially supported by CONICET, FONCyT-ANPCyT, Secyt (UNC), Mincyt (Córdoba). 
$\triangleright$ AAY for braidings of super type;

$\triangleright$ H1, giving a general form of relations for matrices of rank two;

$\triangleright[\mathrm{He}]$ for some examples of rank two matrices, but giving explicit relations.

In A2 we gave general formulae for defining relations of Nichols algebras of diagonal type, see Theorem 1.25 below. The expression of those relations and the proof that they generate the defining ideal are independent of Heckenberger's classification; they rely in Kharchenko's and Rosso's PBW bases $[\mathrm{Kh}, \mathrm{R}$, and a detailed study of convex orders in generalized root systems [A2, through the classification of coideal subalgebras [HS]. In this paper we refine the main result of $\mathrm{A2}$ and prove:

Theorem 1. A minimal set of relations of $\mathcal{B}(V)$ is obtained by considering relations of the following type:

(1) Quantum Serre relations, and powers of generators $x_{i}$ corresponding to non-Cartan vertices; they are needed to introduce Lusztig's isomorphisms at the level of doubles of tensor algebras.

(2) Relations in the image of the previous ones by the Lusztig's isomorphisms, and correspond to relations (23) in Theorem 1.25.

(3) Relations that guarantee that the ideal generated by the previous relations is a braided biideal: they appear in the coproduct of relations of the item (2) in the tensor algebra $T(V)$.

(4) Powers of root vectors (generators of the PBW basis) corresponding to roots in the orbit of Cartan vertices.

See Theorem 3.1 for a complete and explicit set of relations. In this set we distinguish relations appearing in [A2] for standard braidings, and relations in [Y] related with braidings of super type. There exists also a large list of new relations, related with the set of exceptional braidings or with braidings of super type evaluated in roots of unity of small order.

2. The knowledge of the explicit relations of a Nichols algebra has several potential applications to the theory of pointed Hopf algebras, that we discuss now:

- One of the basic question in the Lifting Method AS1, AS3 for the classification of Hopf algebras is the following:

Conjecture 1. AS2, Conjecture 1.4] Let $\Gamma$ be a finite group and $\mathbf{k}$ an algebraically closed field of characteristic 0 . If $H$ is a finite-dimensional pointed Hopf algebra over $\mathbf{k}$ such that $G(H)=\Gamma$, then $H$ is generated as an algebra by $\Gamma$ and its skew-primitive elements.

This question was answered in AS4 for braidings of Cartan type under some mild conditions. This result was extended to the case of standard braidings in [AGI]. In Section [4 we obtain as a consequence of Theorem 3.1.

Theorem 2. Let $H$ be a finite dimensional pointed Hopf algebra over an abelian group $\Gamma$. Then $H$ is generated as an algebra by $\Gamma$ and its skew primitive elements.

That is, we answer positively Conjecture 1 in a general context: when $G(H)$ is any abelian group. This Theorem is also applied to the known cases of finite-dimensional Nichols algebras over non-abelian groups

- Another crucial step of the Lifting Method is to obtain all deformations of the pointed Hopf algebras $\mathcal{B}(V) \# \mathbf{k} \Gamma$; that is, all the pointed Hopf algebras such that their associated coradically graded algebras are isomorphic to $\mathcal{B}(V) \# \mathbf{k} \Gamma$.

This problem was solved for $\Gamma$ abelian in AS4 - under the restriction that the order is not divisible by $2,3,5,7$. We believe that the explicit presentation in this paper would be substantial to solve the question for any abelian group. 
- The explicit relations would be also useful in the study of various elements in the representation theory of pointed Hopf algebras. In this direction, the theory of Nichols algebras of diagonal type provides an uniform approach to the study of quantum groups and quantum supergroups.

3. The plan of this paper is the following. We introduce the notion of Nichols algebras in Section 1. We give a PBW basis of any Nichols algebra and some properties of this basis following $[\mathrm{Kh}, \mathbb{R}$. Next we recall the notions of Weyl groupoid and its associated root system following [CH2, $\mathrm{HS}$ ], and make a connection with the theory of Nichols algebras of diagonal type. We present the needed material from [A2], in particular Theorem 1.25, a key result for our purposes.

Section 2 is devoted to Lusztig's isomorphisms in the general context of braidings of diagonal type [H4], extending analogous isomorphisms from [L].

In Section 3 we give the mentioned presentation by generators and relations, based in the classification of braidings of diagonal type with finite root system [H3]. The strategy of proof consists first to define Lusztig isomorphisms for the Drinfeld doubles of the braided Hopf algebras $U^{+}$obtained by quotient by the relations in Theorem 1, except the group in (4). This quotient is analogous to the algebra $U_{q}^{+}(\mathfrak{g})$; the Drinfeld double $\mathfrak{u}_{q}(\mathfrak{g})$ of the Nichols algebra is a quotient of the previous algebra, as it was considered by Lusztig and Andruskiewitsch-Schneider. We denote these two algebras by $U^{+}$and $\mathfrak{u}^{+}$, respectively, so $\mathfrak{u}^{+}=\mathcal{B}(V)$. The existence of the Lusztig's isomorphisms prove that the PBW generators corresponding to the algebras $U^{+}$and their quotients $\mathfrak{u}^{+}$are the same, but the heights of some generators are not the order of the associated scalar in $U^{+}$. Therefore we obtain $\mathfrak{u}^{+}$ after to quotient $U^{+}$by some powers of root vectors as in (4).

Theorem 3.1 extends the presentation obtained in [A1] for standard braidings, and in AAY for braidings of super type, and gives a new proof in the case of braidings of Cartan type, in particular quantized enveloping algebras $U_{q}(\mathfrak{g})$ and small quantum groups $\mathfrak{u}_{q}(\mathfrak{g})$.

Finally, Section 4 is devoted to the proof of Theorem 2. We prove first that any finite dimensional braided graded Hopf algebra of diagonal type

$$
S=\oplus_{n \geq 0} S_{n}, \quad S_{0}=\mathbf{k} 1, S_{1} \cong V,
$$

generated as an algebra by $V$ is isomorphic to the Nichols algebra $\mathcal{B}(V)$; this result extends [AS4, Thm. 5.5], AGI, Thm. 2.5], but the proof follows the same scheme.

Acknowledges. This work is part of the author's $\mathrm{PhD}$ Thesis. I want to thank specially to my advisor Nicolás Andruskiewitsch for his inspiring guidance, patience and supervision during these years. I want to thank also to my family for all their support, and to Antonela for all her love.

\section{Preliminaries}

In this Section we recall results from different works needed in the sequel. First we consider the existence of PBW bases for Nichols algebras of diagonal type $[\mathrm{Kh}, \mathrm{R}]$, and the rich combinatoric related to them. Next we recall the definitions of Weyl groupoid, the associated root systems and some properties thereof [HS, HY]. We close this Section stating a general presentation of Nichols algebras coming from A2.

1.1. Lyndon words and PBW bases for Nichols algebras of diagonal type. To begin with, we recall the definition of a Nichols algebras and show a characterization in the case of a diagonal braiding. 
Definition 1.1. AS3 Given $V \in_{H}^{H} \mathcal{Y} \mathcal{D}$, the tensor algebra $T(V)$ admits a unique structure of graded braided Hopf algebra in ${ }_{H}^{H} \mathcal{Y} \mathcal{D}$ such that $V \subseteq \mathcal{P}(V)$. Consider the family $\mathfrak{S}$ of all the homogeneous Hopf ideals $I \subseteq T(V)$ such that

- $I$ is generated by homogeneous elements of degree $\geq 2$,

- $I$ is a Yetter-Drinfeld submodule of $T(V)$.

The Nichols algebra $\mathcal{B}(V)$ associated to $V$ is the quotient of $T(V)$ by the biggest ideal $I(V)$ of $\mathfrak{S}$.

Let $(V, c)$ be a braided vector space of diagonal type such that $q_{i j}=q_{j i}$ for any $i, j$. Let $\Gamma=\mathbb{Z}^{\theta}$, and $\alpha_{1}, \ldots, \alpha_{\theta}$ be the canonical basis. We set the characters $\chi_{1}, \ldots, \chi_{\theta}$ of $\Gamma$ given by $\chi_{j}\left(\alpha_{i}\right)=q_{i j}, 1 \leq i, j \leq \theta$.

Consider $V$ as a Yetter-Drinfeld module over $\mathrm{k} \Gamma$ such that $x_{i} \in V_{\alpha_{i}}^{\chi_{i}}$. In this context we can characterize the Nichols algebra as a quotient that admits a certain non-degenerate bilinear form. We use Swedler's notation for the coproduct in $T(V): \Delta(x)=x_{1} \otimes x_{2}$, where we omit the summation symbol.

Proposition 1.2. [L, Prop. 1.2.3], [AS3, Prop. 2.10] There exists a unique bilinear form $(\cdot \mid \cdot): T(V) \times T(V) \rightarrow \mathbf{k}$ such that $(1 \mid 1)=1$, and:

$$
\begin{aligned}
& \left(x_{i} \mid x_{j}\right)=\delta_{i j}, \quad \text { for any } i, j ; \\
& \left(x \mid y y^{\prime}\right)=\left(x_{1} \mid y\right)\left(x_{2} \mid y^{\prime}\right), \quad \text { for any } x, y, y^{\prime} \in T(V) ; \\
& \left(x x^{\prime} \mid y\right)=\left(x \mid y_{1}\right)\left(x^{\prime} \mid y_{2}\right), \quad \text { for any } x, x^{\prime}, y \in T(V) .
\end{aligned}
$$

This is a symmetric form, for which we have:

$$
(x \mid y)=0, \quad \text { for any } x \in T(V)_{g}, y \in T(V)_{h}, g, h \in \Gamma, g \neq h .
$$

The radical of this form $\{x \in T(V):(x \mid y)=0, \forall y \in T(V)\}$ coincides with $I(V)$, so $(\cdot \mid \cdot)$ induces a non-degenerate bilinear form on $\mathcal{B}(V)=T(V) / I(V)$, denoted also by $(\cdot \mid \cdot)$.

Therefore $I(V)$ is a $\mathbb{Z}^{\theta}$-homogeneous ideal, and then $\mathcal{B}(V)$ is $\mathbb{Z}^{\theta}$-graded.

Let $A$ be an algebra, $P, S \subset A$ and $h: S \mapsto \mathbb{N} \cup\{\infty\}$. We fix a linear order $<$ on $S$. $B(P, S,<, h)$ will denote the set

$$
\left\{p s_{1}^{e_{1}} \ldots s_{t}^{e_{t}}: t \in \mathbb{N}_{0}, \quad s_{1}>\cdots>s_{t}, \quad s_{i} \in S, \quad 0<e_{i}<h\left(s_{i}\right), \quad p \in P\right\} .
$$

If $B(P, S,<, h)$ is a k-linear basis, we say that $(P, S,<, h)$ is a set of $P B W$ generators, whose height is $h$, and $B(P, S,<, h)$ is a $P B W$ basis of $A$.

We will describe a particular PBW basis for any graded braided Hopf algebra $\mathcal{B}=$ $\oplus_{n \in \mathbb{N}} \mathcal{B}^{n}$ generated by $\mathcal{B}^{1} \cong V$ as an algebra, where $V$ is a braided vector space; we will follow the results in $\mathrm{Kh}$.

Fix $\theta \in \mathbb{N}$, and a set $X=\left\{x_{1}, \ldots, x_{\theta}\right\}$. Let $\mathbb{X}$ be the set of words with letters in $X$ and consider the lexicographical order on $\mathbb{X}$.

Definition 1.3. An element $u \in \mathbb{X}, u \neq 1$ is a Lyndon word if for any decomposition $u=v w, v, w \in \mathbb{X}-\{1\}$, we have $u<w$. We will denote the set of all Lyndon words by $L$.

Remark 1.4. • Each Lyndon word begin with its smallest letter.

- Each $u \in \mathbb{X}-X$ is a Lyndon word if and only if for each decomposition $u=u_{1} u_{2}$ with $u_{1}, u_{2} \in \mathbb{X} \backslash 1$, we have $u_{1} u_{2}=u<u_{2} u_{1}$.

- If $u_{1}, u_{2} \in L$ and $u_{1}<u_{2}$, then $u_{1} u_{2} \in L$. 
A basic Lyndon's result says that any word $u \in \mathbb{X}$ admits a unique decomposition as non-increasing product of Lyndon words:

$$
u=l_{1} l_{2} \ldots l_{r}, \quad l_{i} \in L, l_{r} \leq \cdots \leq l_{1} .
$$

It is called the Lyndon decomposition of $u \in \mathbb{X}$, and the $l_{i} \in L$ in (5) are called the Lyndon letters of $u$.

Another characterization of Lyndon words is the following:

Lemma 1.5. [Kh, p.6] Let $u \in \mathbb{X}-X$. Then $u \in L$ if and only if there exist $u_{1}, u_{2} \in L$ such that $u_{1}<u_{2}$ and $u=u_{1} u_{2}$.

Definition 1.6. For each $u \in L-X$, the Shirshov decomposition of $u$ is the decomposition $u=u_{1} u_{2}, u_{1}, u_{2} \in L$, such that $u_{2}$ is the smallest end of $u$ between all the possible decompositions with these conditions.

Given a finite-dimensional vector space $V$, fix a basis $X=\left\{x_{1}, \ldots, x_{\theta}\right\}$ of $V$; we can identify $\mathbf{k} \mathbb{X}$ with $T(V)$. In what follows we consider two graduations for the algebra $T(V)$ : the usual $\mathbb{N}_{0}$-graduation $T(V)=\oplus_{n \geq 0} T^{n}(V)$, and $\mathbb{Z}^{\theta}$-graduation of $T(V)$, determined by the condition $\operatorname{deg} x_{i}=\alpha_{i}, 1 \leq i \leq \theta$, where $\left\{\alpha_{1}, \ldots, \alpha_{\theta}\right\}$ is the canonical basis of $\mathbb{Z}^{\theta}$.

Consider a braiding $c$ for $V$. The braided bracket of $x, y \in T(V)$ is defined by

$$
[x, y]_{c}:=\text { multiplication } \circ(\mathrm{id}-c)(x \otimes y) .
$$

Assume that $(V, c)$ is of diagonal type, and let $\chi: \mathbb{Z}^{\theta} \times \mathbb{Z}^{\theta} \rightarrow \mathbf{k}^{\times}$be the bicharacter determined by the condition

$$
\chi\left(\alpha_{i}, \alpha_{j}\right)=q_{i j}, \quad \text { for each pair } 1 \leq i, j \leq \theta .
$$

Then, for each pair of $\mathbb{Z}^{\theta}$-homogeneous elements $u, v \in \mathbb{X}$,

$$
c(u \otimes v)=q_{u, v} v \otimes u, \quad q_{u, v}=\chi(\operatorname{deg} u, \operatorname{deg} v) \in \mathbf{k}^{\times} .
$$

In such case, the braided bracket satisfies a "braided Jacobi identity" and determines skew-derivations as follows:

$$
\begin{aligned}
{\left[[u, v]_{c}, w\right]_{c} } & =\left[u,[v, w]_{c}\right]_{c}-\chi(\alpha, \beta) v[u, w]_{c}+\chi(\beta, \gamma)[u, w]_{c} v \\
{[u, v w]_{c} } & =[u, v]_{c} w+\chi(\alpha, \beta) v[u, w]_{c}, \\
{[u v, w]_{c} } & =\chi(\beta, \gamma)[u, w]_{c} v+u[v, w]_{c},
\end{aligned}
$$

where $u, v, w \in T(V)$ are homogeneous of degree $\alpha, \beta, \gamma \in \mathbb{N}^{\theta}$, respectively.

Using the previous decompositions, we can define the $\mathbf{k}$-linear endomorphism $[-]_{c}$ of $\mathbf{k} \mathbb{X}$ as follows:

$$
[u]_{c}:= \begin{cases}u, & \text { if } u=1 \text { or } u \in X ; \\ {\left[[v]_{c},[w]_{c}\right]_{c},} & \text { if } u \in L, \ell(u)>1, u=v w \text { is the Shirshov decomposition; } \\ {\left[u_{1}\right]_{c} \ldots\left[u_{t}\right]_{c},} & \text { if } u \in \mathbb{X}-L \text { and its Lyndon decomposition is } u=u_{1} \ldots u_{t} .\end{cases}
$$

Definition 1.7. The hyperletter corresponding to $l \in L$ is $[l]_{c}$. An hyperword is a word whose letters are hyperletters, and a monotone hyperword is an hyperword $W=$ $\left[u_{1}\right]_{c}^{k_{1}} \ldots\left[u_{m}\right]_{c}^{k_{m}}$ such that $u_{1}>\cdots>u_{m}$.

Remark 1.8. For any $u \in L,[u]_{c}$ is a $\mathbb{Z}\left[q_{i j}\right]$-linear combination of words with the same $\mathbb{Z}^{\theta}$-graduation than $u$, such that $[u]_{c} \in u+\mathbf{k} \mathbb{X}_{>u}^{\ell(u)}$.

Theorem 1.9. $\left[\mathbb{R}\right.$, Thm. 10] Let $u, v \in L, u<v$. Then $\left[[u]_{c},[v]_{c}\right]_{c}$ is a $\mathbb{Z}\left[q_{i j}\right]$-linear combination of monotone hyperwords $\left[l_{1}\right]_{c} \ldots\left[l_{r}\right]_{c}, l_{i} \in L$, such that the corresponding hyperletters satisfy $v>l_{i} \geq u v$. Moreover, $[u v]_{c}$ appears in such combination with nonzero coefficient and each hyperword has the same $\mathbb{Z}^{\theta}$-graduation than uv. 
We consider the polynomials in $\mathbb{Z}[t]$ :

$$
(n)_{t}:=1+t+\cdots+t^{n-1}, \quad(n)_{t} !=(1)_{t}(2)_{t} \cdots(n)_{t}, \quad n \in \mathbb{N} .
$$

The q-combinatorial numbers are defined as the following quotient:

$$
\left(\begin{array}{c}
n \\
i
\end{array}\right)_{t}:=\frac{(n)_{t} !}{(n-i)_{t} !(i)_{t} !}, \quad 0 \leq i \leq n
$$

It follows inductively that $\left(\begin{array}{c}n \\ i\end{array}\right)_{t} \in \mathbb{Z}[t]$, for all $n$ and all $i \in\{0,1, \ldots, n\}$. Therefore $\left(\begin{array}{c}n \\ i\end{array}\right)_{q}$ will denote the evaluation of $\left(\begin{array}{c}n \\ i\end{array}\right)_{t}$ for $t=q$, where $q \in \mathbf{k}$.

The comultiplication of hyperwords in $T(V)$ has a nice expression, as we can see in the following result.

Lemma 1.10. [R, Thm.13] Let $u_{1}, \ldots, u_{r}, v \in L$, with $v<u_{r} \leq \cdots \leq u_{1}$. Then,

$$
\begin{aligned}
\Delta\left(\left[u_{1}\right]_{c} \cdots\left[u_{r}\right]_{c}[v]_{c}^{m}\right)= & 1 \otimes\left[u_{1}\right]_{c} \cdots\left[u_{r}\right]_{c}[v]_{c}^{m}+\sum_{i=0}^{m}\left(\begin{array}{c}
m \\
i
\end{array}\right)_{q_{v, v}}\left[u_{1}\right]_{c} \ldots\left[u_{r}\right]_{c}[v]_{c}^{i} \otimes[v]_{c}^{m-i} \\
& +\sum_{\substack{l_{1} \geq \cdots \geq l_{p}>v, l_{i} \in L \\
0 \leq j \leq m}} x_{l_{1}, \ldots, l_{p}}^{(j)} \otimes\left[l_{1}\right]_{c} \cdots\left[l_{p}\right]_{c}[v]_{c}^{j}
\end{aligned}
$$

Where $x_{l_{1}, \ldots, l_{p}}^{(j)}$ is $\mathbb{Z}^{\theta}$-homogeneous, and $\operatorname{deg}\left(x_{l_{1}, \ldots, l_{p}}^{(j)}\right)+\operatorname{deg}\left(l_{1} \ldots l_{p} v^{j}\right)=\operatorname{deg}\left(u_{1} \ldots u_{r} v^{m}\right)$.

Another useful result from $[\mathrm{R}]$ is the following one.

Lemma 1.11. For each $l \in L$ let $W_{l}$ be the subspace of $T(V)$ generated by

$$
\left[l_{1}\right]_{c}\left[l_{2}\right]_{c} \cdots\left[l_{k}\right]_{c}, \quad k \in \mathbb{N}_{0}, l_{i} \in L, l_{1} \geq \ldots \geq l_{k} \geq l .
$$

Then $W_{l}$ is a left coideal subalgebra of $T(V)$.

We consider another order in $\mathbb{X}$ as in $[\mathrm{U}]$; it was implicitly used in $[\mathrm{Kh}$. Let $u, v \in \mathbb{X}$. We say that $u \succ v$ if $\ell(u)<\ell(v)$, or $\ell(u)=\ell(v)$ and $u>v$ for the lexicographical order. This order $\succ$ is total, and it is called the deg-lex order.

The empty word 1 is the maximal element for $\succ$, and this order is invariant by left and right multiplication.

Let $I$ be an ideal of $T(V)$, and $R=T(V) / I$. Let $\pi: T(V) \rightarrow R$ be the canonical projection. We set:

$$
G_{I}:=\left\{u \in \mathbb{X}: u \notin \mathbf{k} \mathbb{X}_{\succ u}+I\right\} .
$$

Note that if $u \in G_{I}$ and $u=v w$, then $v, w \in G_{I}$. Therefore each $u \in G_{I}$ is a non-increasing product of Lyndon words of $G_{I}$.

Proposition 1.12. $\mathrm{Kh}, \mathrm{R}$, The set $\pi\left(G_{I}\right)$ is a basis of $R$.

In what follows $I$ will denote a Hopf ideal. Consider the set $S_{I}:=G_{I} \cap L$. Define $h_{I}: S_{I} \rightarrow\{2,3, \ldots\} \cup\{\infty\}$ according to the following condition:

$$
h_{I}(u):=\min \left\{t \in \mathbb{N}: u^{t} \in \mathbf{k} \mathbb{X}_{\succ u^{t}}+I\right\} .
$$

We recall the following result and its corollaries following $[\mathrm{Kh}$.

Theorem 1.13. $B_{I}^{\prime}:=B\left(\{1+I\},\left[S_{I}\right]_{c}+I,<, h_{I}\right)$ is a $P B W$ basis of $H=T(V) / I$.

Corollary 1.14. A word $u$ belongs to $G_{I}$ if and only if the corresponding hyperletter $[u]_{c}$ is not a linear combination, modulo $I$, of greater hyperwords $[w]_{c}, w \succ u$, whose hyperletters are in $S_{I}$. 
Corollary 1.15. If $v \in S_{I}$ is such that $h_{I}(v)<\infty$, then $q_{v, v}$ is a root of unity. Moreover, if $\operatorname{ord} q_{v, v}=h$, then $h_{I}(v)=h$, and $[v]^{h}$ is a linear combination of hyperwords $[w]_{c}$, $w \succ v^{h}$.

1.2. Weyl groupoids and root systems. We follow the notation in [CH1]. Fix a nonempty set $\mathfrak{X}$, and a finite set $I$. For each $i \in I$ we fix a bijective function $r_{i}: \mathfrak{X} \rightarrow \mathfrak{X}$, and for each $X \in \mathfrak{X}$ a generalized Cartan matrix $A^{X}=\left(a_{i j}^{X}\right)_{i, j \in I}$. Let $\left(\alpha_{i}\right)_{i \in I}$ be the canonical basis of $\mathbb{Z}^{I}$.

Definition 1.16. [HY, CH1 The 4 -uple $\mathcal{C}:=\mathcal{C}\left(I, \mathfrak{X},\left(r_{i}\right)_{i \in I},\left(A^{X}\right)_{X \in \mathfrak{X}}\right)$ is a Cartan scheme if it holds:

- for any $i \in I, r_{i}^{2}=i d$, and

- for any $X \in \mathfrak{X}$ and any pair $i, j \in I: a_{i j}^{X}=a_{i j}^{r_{i}(X)}$.

For each $i \in I$ and each $X \in \mathfrak{X}$ we denote by $s_{i}^{X}$ the automorphism of $\mathbb{Z}^{I}$ given by

$$
s_{i}^{X}\left(\alpha_{j}\right)=\alpha_{j}-a_{i j}^{X} \alpha_{i}, \quad j \in I .
$$

The Weyl groupoid of $\mathcal{C}$ is the groupoid $\mathcal{W}(\mathcal{C})$ whose set of objects is $\mathfrak{X}$ and whose morphisms are generated by $s_{i}^{X}$, considered as elements $s_{i}^{X} \in \operatorname{Hom}\left(X, r_{i}(X)\right), i \in I, X \in \mathfrak{X}$.

In general we denote $\mathcal{W}(\mathcal{C})$ simply by $\mathcal{W}$, and for each $X \in \mathfrak{X}$ :

$$
\begin{aligned}
\operatorname{Hom}(\mathcal{W}, X) & :=\cup_{Y \in \mathfrak{X}} \operatorname{Hom}(Y, X), \\
\Delta^{X} r e & :=\left\{w\left(\alpha_{i}\right): i \in I, w \in \operatorname{Hom}(\mathcal{W}, X)\right\} .
\end{aligned}
$$

$\Delta^{X}$ re is the set of real roots of $X$. Each $w \in \operatorname{Hom}\left(\mathcal{W}, X_{1}\right)$ is written as a product $s_{i_{1}}^{X_{2}} s_{i_{2}}^{X_{3}} \cdots s_{i_{m}}^{X_{m+1}}$, where $X_{j}=r_{i_{j-1}} \cdots r_{i_{1}}\left(X_{1}\right), i \geq 2$. We denote it by $w=\operatorname{id}_{X_{1}} s_{i_{1}} \cdots s_{i_{m}}$ : it means that $w \in \operatorname{Hom}\left(\mathcal{W}, X_{1}\right)$, because each $X_{j} \in \mathfrak{X}$ is univocally determined by this condition. The length of $w$ is defined by

$$
\ell(w)=\min \left\{n \in \mathbb{N}_{0}: \exists i_{1}, \ldots, i_{n} \in I \text { such that } w=\operatorname{id}_{X} s_{i_{1}} \cdots s_{i_{n}}\right\} .
$$

We assume that $\mathcal{W}$ is connected: that is, $\operatorname{Hom}(Y, X) \neq \emptyset$, for any pair $X, Y \in \mathfrak{X}$.

Definition 1.17. [HY, CH1 Given a Cartan scheme $\mathcal{C}$, consider for each $X \in \mathfrak{X}$ a set $\Delta^{X} \subset \mathbb{Z}^{I}$. We say that $\mathcal{R}:=\mathcal{R}\left(\mathcal{C},\left(\Delta^{X}\right)_{X \in \mathfrak{X}}\right)$ is a root system of type $\mathcal{C}$ if

(1) for any $X \in \mathfrak{X}, \Delta^{X}=\left(\Delta^{X} \cap \mathbb{N}_{0}^{I}\right) \cup-\left(\Delta^{X} \cap \mathbb{N}_{0}^{I}\right)$,

(2) for any $i \in I$ and any $X \in \mathfrak{X}, \Delta^{X} \cap \mathbb{Z} \alpha_{i}=\left\{ \pm \alpha_{i}\right\}$,

(3) for any $i \in I$ and any $X \in \mathfrak{X}, s_{i}^{X}\left(\Delta^{X}\right)=\Delta^{r_{i}(X)}$,

(4) if $m_{i j}^{X}:=\left|\Delta^{X} \cap\left(\mathbb{N}_{0} \alpha_{i}+\mathbb{N}_{0} \alpha_{j}\right)\right|$, then $\left(r_{i} r_{j}\right)^{m_{i j}^{X}}(X)=X$ for any pair $i \neq j \in I$ and any $X \in \mathfrak{X}$.

$\Delta_{+}^{X}:=\Delta^{X} \cap \mathbb{N}_{0}^{I}$ is called the set of positive roots, and $\Delta_{-}^{X}:=-\Delta_{+}^{X}$ is the set of negative roots.

Remark 1.18. From (2) and (3) we deduce that $\Delta^{X}$ re $\subset \Delta^{X}$, for any $X \in \mathfrak{X}$.

For each positive root $\alpha=\sum_{i} n_{i} \alpha_{i}$, the support of $\alpha$ is the set

$$
\operatorname{supp} \alpha:=\left\{i: 1 \leq i \leq \theta, n_{i} \neq 0\right\} .
$$

By (3) we have that $w\left(\Delta^{X}\right)=\Delta^{Y}$ for any $w \in \operatorname{Hom}(Y, X)$. We say that $\mathcal{R}$ is finite if $\Delta^{X}$ is finite for some $X \in \mathfrak{X}$. By [CH1, Lemma 2.11], it is equivalent to the fact that all the sets $\Delta^{X}$ are finite, $X \in \mathfrak{X}$, and also that $\mathcal{W}$ is finite. Moreover, for any pair $i \neq j \in I$ and any $X \in \mathfrak{X}$, we have that $k \alpha_{i}+\alpha_{j} \in \Delta^{X}$ if and only if $0 \leq k \leq-a_{i j}^{X}$. Therefore,

$$
a_{i j}^{X}=-\max \left\{k \in \mathbb{N}_{0}: k \alpha_{i}+\alpha_{j} \in \Delta^{X}\right\} .
$$


A fundamental result involving root systems is the following one:

Theorem 1.19. [CH2, Thm. 2.10] For every $\alpha \in \Delta_{+}^{X} \backslash\left\{\alpha_{i}: i=1, \ldots \theta\right\}$, there exist $\beta, \gamma \in \Delta_{+}^{X}$ such that $\alpha=\beta+\gamma$.

We give now some results about real roots and the length of elements.

Lemma 1.20. [HY, Cor. 3] Let $m \in \mathbb{N}, X, Y \in \mathfrak{X}, i_{1}, \ldots, i_{m}, j \in I, w=\operatorname{id}_{X} s_{i_{1}} \cdots s_{i_{m}} \in$ $\operatorname{Hom}(Y, X)$, where $\ell(w)=m$. Then,

- $\ell\left(w s_{j}\right)=m+1$ if and only if $w\left(\alpha_{j}\right) \in \Delta_{+}^{X}$,

- $\ell\left(w s_{j}\right)=m-1$ if and only if $w\left(\alpha_{j}\right) \in \Delta_{-}^{X}$.

Proposition 1.21. CH1, Prop. 2.12] For any $w=\mathrm{id}_{X} s_{i_{1}} \cdots s_{i_{m}} \in \mathcal{W}$ such that $\ell(w)=$ $m$, the roots $\beta_{j}=s_{i_{1}} \cdots s_{i_{j-1}}\left(\alpha_{i_{j}}\right) \in \Delta^{X}$ are positive and all different. If $\mathcal{R}$ is finite and $w$ is an element of maximal length, say $N$, then $\Delta_{+}^{X}=\left\{\beta_{j} \mid 1 \leq j \leq N\right\}$. The roots $\beta_{1}, \ldots, \beta_{N}$ are pairwise different, and hence for each $\alpha \in \Delta_{+}^{X}$ there exist $i_{1}, \ldots, i_{k}, j \in I$ such that $\alpha=s_{i_{k}} \cdots s_{i_{1}}\left(\alpha_{j}\right)$.

Call $\Delta_{+}^{V}$ the set of degrees of a PBW basis of $\mathcal{B}(V)$, counted with their multiplicities, as in [H2]. It does not depend on the PBW basis, see [H2, AA]. We can attach a Cartan scheme $\mathcal{C}$, a Weyl groupoid $\mathcal{W}$ and a root system $\mathcal{R}$, see [HS, Thms. 6.2, 6.9]. To do this, define for each $1 \leq i \neq j \leq \theta$,

$$
-a_{i j}:=\min \left\{n \in \mathbb{N}_{0}:(n+1)_{q_{i i}}\left(1-q_{i i}^{n} q_{i j} q_{j i}\right)=0\right\},
$$

and set $a_{i i}=2, s_{i} \in \operatorname{Aut}\left(\mathbb{Z}^{\theta}\right)$ such that $s_{i}\left(\alpha_{j}\right)=\alpha_{j}-a_{i j} \alpha_{i}$.

Set $\bar{q}_{r s}=\chi\left(s_{i}\left(\alpha_{r}\right), s_{i}\left(\alpha_{s}\right)\right)$. Let $V_{i}$ be another vector space of dimension $\theta$, and attach to it the matrix $\overline{\mathbf{q}}=\left(\bar{q}_{r s}\right)$. By [H2], $\Delta_{+}^{V_{i}}=s_{i}\left(\Delta_{+}^{V} \backslash\left\{\alpha_{i}\right\}\right) \cup\left\{\alpha_{i}\right\}$. If we consider $\Delta^{V}=$ $\Delta_{+}^{V} \cup\left(-\Delta_{+}^{V}\right)$, last equation lets us to define the Weyl groupoid of $V$, whose root system is defined by the sets $\Delta^{V^{\prime}}, V^{\prime}$ obtained after to apply some reflections to the matrix of $V$.

\subsection{Defining relations of Nichols algebras of diagonal type.}

Proposition 1.22. [A2, Prop. 3.1] Assume that the braiding matrix is symmetric. Then a $P B W$ basis of Lyndon hyperwords of $\mathcal{B}(V)$ is orthogonal with respect to the bilinear form in Proposition 1.2.

Corollary 1.23. [A2, Cor. 3.2] If $u=x_{\beta_{M}}^{n_{M}} \cdots x_{\beta_{1}}^{n_{1}}$, where $0 \leq n_{j}<N_{\beta_{j}}$, then

$$
c_{u}:=(u \mid u)=\prod_{j=1}^{M} n_{j} !_{\beta_{\beta_{j}}} c_{x_{\beta_{j}}}^{n_{j}} \neq 0 .
$$

Remark 1.24. Notice that:

$$
\left(x_{\beta_{i}} x_{\beta_{j}} \mid u\right)=\left(x_{\beta_{i}} \mid u_{(1)}\right)\left(x_{\beta_{j}} \mid u_{(2)}\right)=d_{i, j} c_{x_{\beta_{i}}} c_{x_{\beta_{j}}},
$$

where $d_{i, j}$ is the coefficient $x_{\beta_{i}} \otimes x_{\beta_{j}}$ for the expression of $\Delta(u)$ in terms of the PBW basis (both factors of the tensor product).

For each pair $1 \leq i \leq j \leq \theta$, we denote

$$
B_{i j}:=\left\{x_{\beta_{j}}^{n_{j}} \cdots x_{\beta_{i}}^{n_{i}}: 0 \leq n_{k}<N_{\beta_{k}}\right\} ;
$$

that is, the set of hyperwords whose hyperletters are between $x_{\beta_{i}}$ and $x_{\beta_{j}}$ 
Let $(W, d)$ be a braided vector space of diagonal type that admits a basis $\widehat{x}_{1}, \ldots, \widehat{x}_{\theta}$ such that, for some $\widehat{q}_{i j} \in \mathbf{k}^{\times}, d\left(\widehat{x}_{i} \otimes \widehat{x}_{j}\right)=\widehat{q}_{i j} \widehat{x}_{j} \otimes \widehat{x}_{i}$, where $\widehat{q}_{i j}=\widehat{q}_{j i}$, and $(V, c),(W, d)$ are twist equivalent:

$$
q_{i j} q_{j i}=\widehat{q}_{i j} \widehat{q}_{j i}, \quad q_{i i}=\widehat{q}_{i i}, \quad 1 \leq i \neq j \leq \theta .
$$

Call $\widehat{x}_{\beta}=\left[l_{\beta}\right]_{d}$, the hyperletter corresponding to $l_{\beta}$ for the braiding $d$. If $u=x_{\beta_{M}}^{n_{M}} \cdots x_{\beta_{1}}^{n_{1}}$, call also

$$
\widehat{u}=\widehat{x}_{\beta_{M}}^{n_{M}} \cdots \widehat{x}_{\beta_{1}}^{n_{1}} \text {. }
$$

Let $\sigma: \mathbb{Z}^{\theta} \times \mathbb{Z}^{\theta} \rightarrow \mathbf{k}^{\times}$be the bicharacter determined by the condition

$$
\sigma\left(\alpha_{i}, \alpha_{j}\right)= \begin{cases}\widehat{q}_{i j} q_{i j}^{-1}, & i \leq j \\ 1, & i>j\end{cases}
$$

Define $t_{\alpha_{i}}=1$ for any $1 \leq i \leq \theta$, and inductively,

$$
t_{\beta}=\sigma\left(\beta_{1}, \beta_{2}\right) t_{\beta_{1}} t_{\beta_{2}}, \quad \operatorname{Sh}\left(l_{\beta}\right)=\left(l_{\beta_{1}}, l_{\beta_{2}}\right) .
$$

For each $u=x_{\beta_{M}}^{n_{M}} \cdots x_{\beta_{1}}^{n_{1}}$ call

$$
f(u):=\prod_{1 \leq i<j \leq M} \sigma\left(\beta_{j}, \beta_{i}\right)^{n_{i} n_{j}} \prod_{1 \leq i \leq M} \sigma\left(\beta_{i}, \beta_{i}\right)^{\left(\begin{array}{c}
n_{i} \\
2
\end{array}\right)} t_{\beta_{i}}^{n_{i}} .
$$

Finally, for each pair $1 \leq i<j \leq \theta$ and $u=x_{\beta_{M}}^{n_{M}} \cdots x_{\beta_{1}}^{n_{1}}$, let

$$
c_{i, j}^{u}:=\frac{f(u)\left(\widehat{x}_{\beta_{i}} \widehat{x}_{\beta_{j}} \mid \widehat{u}\right)}{\sigma\left(\beta_{i}, \beta_{j}\right) t_{\beta_{i}} t_{\beta_{j}} c_{\widehat{u}}},
$$

where $(\cdot \mid \cdot)$ is the bilinear form corresponding to $(W, d)$, and $c_{\widehat{u}}$ is the scalar in Corollary 1.23. Such scalars let us to give a presentation by generators and relations as follows.

Theorem 1.25. A2, Thm. 3.9] Let $(V, c)$ be a finite-dimensional braided vector space of diagonal type such that $\Delta_{+}^{V}$ is finite. Let $x_{1}, \cdots, x_{\theta}$ be a basis of $V$ such that $c\left(x_{i} \otimes x_{j}\right)=$ $q_{i j} x_{j} \otimes x_{i}$, where $\left(q_{i j}\right) \in\left(\mathbf{k}^{\times}\right)^{\theta \times \theta}$ is the braiding matrix, and let $\left\{x_{\beta_{k}}\right\}_{\beta_{k} \in \Delta_{+}^{V}}$ be the set of hyperletters corresponding to the fixed order of the basis of $V$.

Then $\mathcal{B}(V)$ is presented by generators $x_{1}, \ldots, x_{\theta}$, and relations

$$
\begin{aligned}
x_{\beta}^{N_{\beta}} & =0, \quad \beta \in \Delta_{+}^{V}, \operatorname{ord}\left(q_{\beta}\right)=N_{\beta}<\infty, \\
{\left[x_{\beta_{i}}, x_{\beta_{j}}\right]_{c} } & =\sum_{u \in B_{i j}-\left\{x_{\beta_{j}} x_{\beta_{i}}\right\}: \operatorname{deg} u=\beta_{i}+\beta_{j}} c_{i, j}^{u} u, \\
1 & \leq i<j \leq M, \operatorname{Sh}\left(l_{\beta_{i}} l_{\beta_{j}}\right)=\left(l_{\beta_{i}}, l_{\beta_{j}}\right), l_{\beta_{i}} l_{\beta_{j}} \neq l_{\beta_{k}}, \forall k,
\end{aligned}
$$

where $c_{i, j}^{u}$ are as in (21). Moreover, $\left\{x_{\beta_{M}}^{n_{M}} \cdots x_{\beta_{1}}^{n_{1}}: 0 \leq n_{j}<N_{\beta_{j}}\right\}$ is a basis of $\mathcal{B}(V)$.

\section{Lusztig IsOmorphisms of Nichols ALgEBras OF DiAgONAL TYPe}

In this Section we recall the Lusztig isomorphisms $\underline{\mathrm{H} 4}$ of Nichols algebras of diagonal type, which are a generalization of the isomorphisms of quantized enveloping algebras in $[\mathrm{L}$. We shall consider different quotients of the tensor algebra of a braided vector space of diagonal type and the Drinfeld doubles of their bosonizations by a free abelian group.

Notation: Let $\chi: \mathbb{Z}^{\theta} \times \mathbb{Z}^{\theta} \rightarrow \mathbf{k}^{\times}$be a bicharacter, $q_{i j}=\chi\left(\alpha_{i}, \alpha_{j}\right)$. Then $\chi^{\text {op }}$ and $\chi^{-1}$ will denote the bicharacters:

$$
\chi^{o p}(\alpha, \beta):=\chi(\beta, \alpha), \quad \chi^{-1}(\alpha, \beta):=\chi(\alpha, \beta)^{-1}, \quad \alpha, \beta \in \mathbb{Z}^{\theta} .
$$


Also, for any automorphism $s: \mathbb{Z}^{\theta} \rightarrow \mathbb{Z}^{\theta}, s^{*} \chi$ will denote the bicharacter defined by

$$
\left(s^{*} \chi\right)(\alpha, \beta):=\chi\left(s^{-1}(\alpha), s^{-1}(\beta)\right), \quad \alpha, \beta \in \mathbb{Z}^{\theta} .
$$

Let $(V, c)$ a braided vector space of diagonal type, whose braiding matrix is $\left(q_{i j}\right)$. We consider $T(V)$ as an algebra in the category of Yetter-Drinfeld modules over $\mathbf{k} \mathbb{Z}^{\theta}$ as above. We follow the results in [H4, Section 4.1].

Definition 2.1. The Drinfeld double $\mathcal{U}(\chi)$ of the Hopf algebra $T(V) \# \mathbf{k} \mathbb{Z}^{\theta}$ is the algebra generated by elements $E_{i}, F_{i}, K_{i}, K_{i}^{-1}, L_{i}, L_{i}^{-1}, 1 \leq i \leq \theta$, and relations

$$
\begin{array}{lr}
X Y=Y X, \quad X, Y \in\left\{K_{i}^{ \pm}, L_{i}^{ \pm}: 1 \leq i \leq \theta\right\}, & \\
K_{i} K_{i}^{-1}=L_{i} L_{i}^{-1}=1, & L_{i} E_{j} L_{i}^{-1}=q_{j i}^{-1} E_{j}, \\
K_{i} E_{j} K_{i}^{-1}=q_{i j} E_{j}, & L_{i} F_{j} L_{i}^{-1}=q_{j i} F_{j}, \\
K_{i} F_{j} K_{i}^{-1}=q_{i j}^{-1} F_{j}, & \\
E_{i} F_{j}-F_{j} E_{i}=\delta_{i, j}\left(K_{i}-L_{i}\right) . &
\end{array}
$$

It admits a Hopf algebra structure, where the comultiplication satisfies

$$
\begin{array}{lr}
\Delta\left(K_{i}\right)=K_{i} \otimes K_{i}, & \Delta\left(E_{i}\right)=E_{i} \otimes 1+K_{i} \otimes E_{i}, \\
\Delta\left(L_{i}\right)=L_{i} \otimes L_{i}, & \Delta\left(F_{i}\right)=F_{i} \otimes L_{i}+1 \otimes F_{i},
\end{array}
$$

and then $\varepsilon\left(K_{i}\right)=\varepsilon\left(L_{i}\right)=1, \varepsilon\left(E_{i}\right)=\varepsilon\left(F_{i}\right)=0$.

Notice that $\mathcal{U}(\chi)$ is a $\mathbb{Z}^{\theta}$-graded Hopf algebra, where the graduation is characterized by the following conditions:

$$
\operatorname{deg}\left(K_{i}\right)=\operatorname{deg}\left(L_{i}\right)=0, \quad \operatorname{deg}\left(E_{i}\right)=\alpha_{i}, \quad \operatorname{deg}\left(F_{i}\right)=-\alpha_{i} .
$$

$\mathcal{U}^{+}(\chi)$ (respectively, $\mathcal{U}^{-}(\chi)$ ) denotes the subalgebra generated by $E_{i}$ (respectively, $\left.F_{i}\right), 1 \leq i \leq \theta, \mathcal{U}^{+0}(\chi)$ (respectively, $\mathcal{U}^{-0}(\chi)$ ) is the subalgebra generated by $K_{i}, K_{i}^{-1}$ (respectively, $L_{i}, L_{i}^{-1}$ ), $1 \leq i \leq \theta$, and finally $\mathcal{U}^{0}(\chi)$ is the subalgebra generated by $K_{i}$, $K_{i}^{-1}, L_{i}$ and $L_{i}^{-1}$. Note that $\mathcal{U}^{0}(\chi)$ is isomorphic to $\mathbf{k} \mathbb{Z}^{2 \theta}$ as Hopf algebras. Moreover, the subalgebra generated by $\mathcal{U}^{+}(\chi), K_{i}$ and $K_{i}^{-1}, 1 \leq i \leq \theta$, is isomorphic to $T(V) \# \mathbf{k} \mathbb{Z}^{\theta}$, so $\mathcal{U}^{+}(\chi)$ is isomorphic to $T(V)$ as braided graded Hopf algebras in the category of YetterDrinfeld modules over $\mathbf{k} \mathbb{Z}^{\theta}$, where we consider the actions and coactions:

$$
K_{i} \cdot E_{j}=q_{i j} E_{j}, \quad \delta\left(E_{i}\right)=K_{i} \otimes E_{i} .
$$

We will consider a family of useful isomorphisms as in [H4, Section 4.1].

Proposition 2.2. (a) For any $\underline{a}=\left(a_{1}, \ldots, a_{\theta}\right) \in\left(\mathbf{k}^{\times}\right)^{\theta}$ there exists a unique algebra automorphism $\varphi_{\underline{a}}$ of $\mathcal{U}(\chi)$ such that

$$
\varphi_{\underline{a}}\left(K_{i}\right)=K_{i}, \quad \varphi_{\underline{a}}\left(L_{i}\right)=L_{i}, \quad \varphi_{\underline{a}}\left(E_{i}\right)=a_{i} E_{i}, \quad \varphi_{\underline{a}}\left(F_{i}\right)=a_{i}^{-1} F_{i} .
$$

(b) There exists a unique algebra automorphism $\phi_{1}$ of $\mathcal{U}(\chi)$ such that

$$
\phi_{1}\left(K_{i}\right)=K_{i}^{-1}, \quad \phi_{1}\left(L_{i}\right)=L_{i}^{-1}, \quad \phi_{1}\left(E_{i}\right)=F_{i} L_{i}^{-1}, \quad \phi_{1}\left(F_{i}\right)=K_{i}^{-1} E_{i} .
$$

(c) There exists a unique algebra isomorphism $\phi_{2}: \mathcal{U}(\chi) \rightarrow \mathcal{U}\left(\chi^{-1}\right)$ such that

$$
\phi_{2}\left(K_{i}\right)=K_{i}, \quad \phi_{2}\left(L_{i}\right)=L_{i}, \quad \phi_{2}\left(E_{i}\right)=F_{i}, \quad \phi_{2}\left(F_{i}\right)=-E_{i} .
$$

(d) There exists a unique Hopf algebra isomorphism $\phi_{3}: \mathcal{U}(\chi) \rightarrow \mathcal{U}\left(\chi^{\mathrm{op}}\right)^{\mathrm{cop}}$ such that

$$
\phi_{3}\left(K_{i}\right)=L_{i}, \quad \phi_{3}\left(L_{i}\right)=K_{i}, \quad \phi_{3}\left(E_{i}\right)=F_{i}, \quad \phi_{3}\left(F_{i}\right)=E_{i} .
$$


(e) There exists a unique algebra antiautomorphism $\phi_{4}$ of $\mathcal{U}(\chi)$ such that

$$
\left.\phi_{4}\left(K_{i}\right)=K_{i}, \quad \phi_{4}\left(L_{i}\right)=L_{i}, \quad \phi_{4}\left(E_{i}\right)=F_{i}, \quad \phi_{(} F_{i}\right)=E_{i} .
$$

(f) Let $\underline{a}=(-1, \cdots,-1)$. The antipode $\mathcal{S}$ of $\mathcal{U}(\chi)$ is given by the composition $\mathcal{S}=$ $\phi_{1} \phi_{4} \varphi_{\underline{a}}$. Also, $\phi_{4}^{2}=\mathrm{id}$.

As in [H4] we will consider some skew-derivations. $\triangleq$ will denote the braided comul-

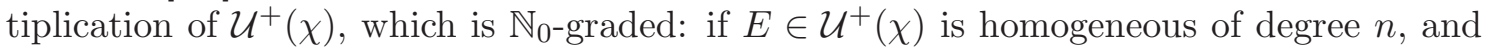
$k \in\{0,1, \ldots, n\}, \underline{\Delta}_{n-k, k}(E)$ will denote the component of $\underline{\Delta}(E)$ in $\mathcal{U}^{+}(\chi)_{n-k} \otimes \mathcal{U}^{+}(\chi)_{k}$.

Proposition 2.3. For any $i \in\{1, \ldots, \theta\}$ there exist linear endomorphisms $\partial_{i}^{K}, \partial_{i}^{L}$ of $\mathcal{U}^{+}(\chi)$ such that

$$
E F_{i}-F_{i} E=\partial_{i}^{K}(E) K_{i}-L_{i} \partial_{i}^{L}(E) \quad \text { for all } E \in \mathcal{U}^{+}(\chi) .
$$

Such endomorphisms are given by:

$$
\underline{\Delta}_{n-1,1}(E)=\sum_{i=1}^{\theta} \partial_{i}^{K}(E) \otimes E_{i}, \quad \underline{\Delta}_{1, n-1}(E)=\sum_{i=1}^{\theta} E_{i} \otimes \partial_{i}^{L}(E), \quad E \in \mathcal{U}^{+}(\chi)_{n},
$$

and satisfy the following conditions:

$$
\begin{aligned}
\partial_{i}^{K}(1) & =\partial_{i}^{L}(1)=0, \\
\partial_{i}^{K}\left(E_{j}\right) & =\partial_{i}^{L}\left(E_{j}\right)=\delta_{i, j}, \\
\partial_{i}^{K}\left(E E^{\prime}\right) & =\partial_{i}^{K}(E)\left(K_{i} \cdot E^{\prime}\right)+E \partial_{i}^{K}\left(E^{\prime}\right), \\
\partial_{i}^{L}\left(E E^{\prime}\right) & =\partial_{i}^{L}(E) E^{\prime}+\left(L_{i}^{-1} \cdot E\right) \partial_{i}^{L}\left(E^{\prime}\right),
\end{aligned}
$$

for all $j \in\{1, \ldots, \theta\}$, and all pair of elements $E, E^{\prime} \in \mathcal{U}^{+}(\chi)$.

We recall now a characterization of quotients of the algebra $\mathcal{U}(\chi)$ with a triangular decomposition [H4, Section 4.1]. According to [H4, Prop. 4.14], the multiplication

$$
m: \mathcal{U}^{+}(\chi) \otimes \mathcal{U}^{0}(\chi) \otimes \mathcal{U}^{-}(\chi) \rightarrow \mathcal{U}(\chi)
$$

is an isomorphism of $\mathbb{Z}^{\theta}$-graded vector spaces.

Proposition 2.4. Let $\mathcal{I}^{+} \subset \mathcal{U}^{+} \cap \operatorname{ker} \varepsilon$ (respectively, $\mathcal{I}^{-} \subset \mathcal{U}^{-} \cap \operatorname{ker} \varepsilon$ ) be an ideal of $\mathcal{U}^{+}(\chi)$ (respectively, $\mathcal{U}^{-}(\chi)$ ). The following conditions are equivalent:

- The multiplication (30) induces an isomorphism

$$
m: \mathcal{U}^{+}(\chi) / \mathcal{I}^{+} \otimes \mathcal{U}^{0}(\chi) \otimes \mathcal{U}^{-}(\chi) / \mathcal{I}^{-} \rightarrow \mathcal{U}(\chi) /\left(\mathcal{I}^{+}+\mathcal{I}^{-}\right) .
$$

- The vector spaces $\mathcal{I}^{+} \mathcal{U}^{0}(\chi) \mathcal{U}^{-}(\chi)$ and $\mathcal{U}^{+}(\chi) \mathcal{U}^{0}(\chi) \mathcal{I}^{-}$are ideals of $\mathcal{U}(\chi)$.

- For all $X \in \mathcal{U}^{0}(\chi)$ and all $i \in\{1, \ldots, \theta\}$ we have

$$
\begin{array}{lll}
X \cdot \mathcal{I}^{+} \subset \mathcal{I}^{+}, & \partial_{i}^{K}\left(\mathcal{I}^{+}\right) \subset \mathcal{I}^{+}, & \partial_{i}^{K}\left(\phi_{4}\left(\mathcal{I}^{-}\right)\right) \subset \phi_{4}\left(\mathcal{I}^{-}\right), \\
X \cdot \mathcal{I}^{-} \subset \mathcal{I}^{-}, & \partial_{i}^{L}\left(\mathcal{I}^{+}\right) \subset \mathcal{I}^{+}, & \partial_{i}^{L}\left(\phi_{4}\left(\mathcal{I}^{-}\right)\right) \subset \phi_{4}\left(\mathcal{I}^{-}\right) .
\end{array}
$$

Lemma 2.5. [H4, Cor. 4.20] Let $I^{+}$be an braided biideal of $\mathcal{U}^{+}(\chi)$, which is also a Yetter-Drinfeld $\mathcal{U}^{0}(\chi)$-submodule and satisfies $I^{+} \subset \oplus_{n \geq 2} \mathcal{U}^{+}(\chi)_{n}$. Then $I^{+} \mathcal{U}^{0}(\chi) \mathcal{U}^{-}(\chi)$ is a Hopf ideal of $\mathcal{U}(\chi)$.

We will assume that all the integers $m_{i j}:=-a_{i j}$ of (17) associated with the matrices $\left(q_{i j}\right)$ are defined. Then we consider the automorphisms $s_{p, \chi}: \mathbb{Z}^{\theta} \rightarrow \mathbb{Z}^{\theta}$. We define also the scalars

$$
\lambda_{i}(\chi):=\left(-a_{p i}\right)_{q_{p p}} \prod_{s=0}^{-a_{p i}-1}\left(q_{p p}^{s} q_{p i} q_{i p}-1\right), \quad i \neq p .
$$


Denote by $\underline{E}_{i}, \underline{F}_{i}, \underline{K}_{i}, \underline{L}_{i}$ the generators corresponding to $\mathcal{U}\left(s_{p}^{*} \chi\right)$, and by $\underline{q}_{i j}=s_{p}^{*} \chi\left(\alpha_{i}, \alpha_{j}\right)$ the coefficients of the braiding matrix corresponding to $s_{p}^{*} \chi$.

Definition 2.6. We say that $p \in\{1, \ldots, \theta\}$ is a Cartan vertex if it satisfies

$$
q_{p p}^{-a_{p j}} q_{p j} q_{j p}=1, \quad \text { for every } j \neq p .
$$

In such case, note that the existence of the integers $-a_{p j}$ implies that ord $q_{p p} \geq-a_{p j}+1$.

We denote by $\mathcal{O}(\chi)$ the union of the orbits of the simple roots $\alpha_{p}$ by the action of the Weyl groupoid, where $p$ is a Cartan vertex.

Fix $p \in\{1, \ldots, \theta\}$. For any $i \neq p$ we define as in [H4],

$$
E_{i, 0(p)}^{+}, E_{i, 0(p)}^{-}:=E_{i}, \quad F_{i, 0(p)}^{+}, F_{i, 0(p)}^{-}:=F_{i},
$$

and recursively,

$$
\begin{aligned}
E_{i, m+1(p)}^{+} & :=E_{p} E_{i, m(p)}^{+}-\left(K_{p} \cdot E_{i, m(p)}^{+}\right) E_{p}=\left(\operatorname{ad}_{c} E_{p}\right)^{m+1} E_{i}, \\
E_{i, m+1(p)}^{-} & :=E_{p} E_{i, m(p)}^{-}-\left(L_{p} \cdot E_{i, m(p)}^{-}\right) E_{p}, \\
F_{i, m+1(p)}^{+} & :=F_{p} F_{i, m(p)}^{+}-\left(L_{p} \cdot F_{i, m(p)}^{+}\right) F_{p}, \\
F_{i, m+1(p)}^{-} & :=F_{p} F_{i, m(p)}^{-}-\left(K_{p} \cdot F_{i, m(p)}^{-}\right) F_{p} .
\end{aligned}
$$

When $p$ is explicit, we simply denote $E_{i, m(p)}^{ \pm}$by $E_{i, m}^{ \pm}$. By [ㅍ4, Cor. 5.4] the following identity holds for any $m \in \mathbb{N}_{0}$ :

$$
E_{i, m}^{+} F_{i}-F_{i} E_{i, m}^{+}=(m)_{q_{p p}}\left(q_{p p}^{m-1} q_{p i} q_{i p}-1\right) L_{p} E_{i, m-1}^{+} .
$$

Fix a braided graded Hopf algebra $\mathcal{B} \cong T(V) / I$, where $I$ is a graded Hopf ideal generated by homogeneous elements of degree $\geq 2$. For each $1 \leq j \leq \theta, p \neq j$, we define

$$
M_{p, j}^{ \pm}(\mathcal{B}):=\left\{E_{j, m}^{ \pm}: m \in \mathbb{N}_{0}\right\} .
$$

In what follows we consider ord $(1)=1$.

Remark 2.7. If $E_{i}^{N}=0$ in $\mathcal{B}$, with $N$ minimal (it is called the nilpotency order of $x_{i}$ ), then $q_{i i}$ is a primitive root of unity of order $N$. Moreover, $\left(\operatorname{ad}_{c} E_{i}\right)^{N} E_{j}=0$.

Also, the nilpotency order of $E_{i}$ is infinite if $E_{i}^{n} \neq 0$ for all $n \in \mathbb{N}$.

We recall a result from [A1] extending [H2, Prop. 1, Eqn. (18)].

Lemma 2.8. For each $p \in\{1, \ldots, \theta\}$, let $\mathcal{B}_{ \pm p}$ be the subalgebra of $\mathcal{B}$ generated by $\cup_{j \neq p} M_{p, j}^{ \pm}(\mathcal{B})$, and denote $N_{p}=$ ord $q_{p p}$. There exist isomorphisms of graded vector spaces:

- $\operatorname{ker}\left(\partial_{p}^{K}\right) \cong \mathcal{B}_{+p} \otimes \mathbf{k}\left[E_{p}^{N_{p}}\right], \operatorname{ker}\left(\partial_{p}^{L}\right) \cong \mathcal{B}_{-p} \otimes \mathbf{k}\left[E_{p}^{N_{p}}\right]$, if $1<\operatorname{ord}\left(q_{p p}\right)<\infty$ but $E_{p}$ is not nilpotent, or

- $\operatorname{ker}\left(\partial_{p}^{K}\right) \cong \mathcal{B}_{+p}, \operatorname{ker}\left(\partial_{p}^{L}\right) \cong \mathcal{B}_{-p}$, if $\operatorname{ord}\left(q_{p p}\right)$ is the nilpotency order of $E_{p}$ or $q_{p p}=1$.

Moreover, the multiplication induces an isomorphism of graded vector spaces $\mathcal{B} \cong \mathcal{B}_{p}^{ \pm} \otimes$ $\mathbf{k}\left[E_{p}\right]$.

Set $N_{p}=\operatorname{ord} q_{p p}$. We call, following [H4], $\mathcal{I}_{p}^{+}(\chi)$ (respectively, $\left.\mathcal{I}_{p}^{-}(\chi)\right)$ to the ideal of $\mathcal{U}^{+}(\chi)$ (respectively, $\left.\mathcal{U}^{-}(\chi)\right)$ generated by

(a) $E_{p}^{N_{p}}$ (respectively, $F_{p}^{N_{p}}$ ), if $p$ is not a Cartan vertex,

(b) $E_{i, m_{p i}+1}^{+}$(respectively, $F_{i, m_{p i}+1}^{+}$), for each $i$ such that $N_{p}>m_{p i}+1$. 
Notice that $E_{i, m_{p i}+1}^{+} \in \mathcal{I}_{p}^{+}(\chi)$ for any $i$ such that $N_{p}=m_{p i}+1$. We denote:

$$
\mathcal{U}_{p}(\chi):=\mathcal{U}(\chi) /\left(\mathcal{I}_{p}^{+}(\chi)+\mathcal{I}_{p}^{-}(\chi)\right), \quad \mathcal{U}_{p}^{+}(\chi):=\mathcal{U}^{+}(\chi) / \mathcal{I}_{p}^{+}(\chi), \quad \mathcal{U}_{p}^{-}(\chi):=\mathcal{U}^{-}(\chi) / \mathcal{I}_{p}^{-}(\chi) .
$$

$I^{+}(\chi)$ will denote the ideal of $\mathcal{U}^{+}(\chi)$ such that the quotient $\mathcal{U}^{+}(\chi) / I^{+}(\chi)$ is isomorphic to the Nichols algebra of $V$; that is, the greatest braided Hopf ideal of $\mathcal{U}^{+}(\chi)$ generated by elements of degree $\geq 2$. Call $I^{-}(\chi)=\phi_{4}\left(I^{+}(\chi)\right)$, where $\phi_{4}$ is defined by (29), and

$$
\mathfrak{u}^{ \pm}(\chi):=\mathcal{U}^{ \pm}(\chi) / I^{ \pm}(\chi), \quad \mathfrak{u}(\chi):=\mathcal{U}(\chi) /\left(I^{-}(\chi)+I^{+}(\chi)\right) .
$$

In such case, $\mathfrak{u}(\chi)$ is the Drinfeld double of the algebra $\mathfrak{u}^{+}(\chi) \# \mathbf{k} \mathbb{Z}^{\theta}$, where $\mathbf{k} \mathbb{Z}^{\theta}=\mathcal{U}^{+0}(\chi)$.

The Lusztig isomorphisms can be defined in this general context.

Theorem 2.9. [H4, Lemma 6.5, Theorem 6.12] There exist algebra morphisms

$$
T_{p}, T_{p}^{-}: \mathcal{U}_{p}(\chi) \rightarrow \mathcal{U}_{p}\left(s_{p}^{*} \chi\right)
$$

univocally determined by the following conditions:

$$
\begin{array}{lr}
T_{p}\left(K_{p}\right)=T_{p}^{-}\left(K_{p}\right)=\underline{K}_{p}^{-1}, & T_{p}\left(K_{i}\right)=T_{p}^{-}\left(K_{i}\right)=\underline{K}_{p}^{m_{p i}} \underline{K}_{i}, \\
T_{p}\left(L_{p}\right)=T_{p}^{-}\left(L_{p}\right)=\underline{L}_{p}^{-1}, & T_{p}\left(L_{i}\right)=T_{p}^{-}\left(L_{i}\right)=\underline{L}_{p}^{m_{p i}} \underline{L}_{i}, \\
T_{p}\left(E_{p}\right)=\underline{F}_{p} \underline{L}_{p}^{-1}, & T_{p}\left(E_{i}\right)=\underline{E}_{i, m_{p i}}^{+}, \\
T_{p}\left(F_{p}\right)=\underline{K}_{p}^{-1} \underline{E}_{p}, & T_{p}\left(F_{i}\right)=\lambda_{p}\left(s_{p}^{*} \chi\right)^{-1} \underline{F}_{i, m_{p i}}^{+}, \\
T_{p}^{-}\left(E_{p}\right)=\underline{K}_{p}^{-1} \underline{F}_{p}, & T_{p}^{-}\left(E_{i}\right)=\lambda_{p}\left(s_{p}^{*} \chi^{-1}\right)^{-1} \underline{E}_{i, m_{p i}}^{-}, \\
T_{p}^{-}\left(F_{p}\right)=\underline{E}_{p} \underline{L}_{p}^{-1}, & T_{p}^{-}\left(F_{i}\right)=\underline{F}_{i, m_{p i}}^{-} .
\end{array}
$$

for every $i \neq p$. Both are isomorphisms satisfying

$$
T_{p} T_{p}^{-}=T_{p}^{-} T_{p}=\mathrm{id}, \quad T_{p}\left(\mathcal{U}_{+p}^{+}(\chi)\right)=\mathcal{U}_{-p}^{+}\left(s_{p}^{*} \chi\right) .
$$

Moreover, there exists $\mu \in\left(\mathbf{k}^{\times}\right)^{\theta}$ such that

$$
T_{p} \circ \phi_{4}=\phi_{4} \circ T_{p}^{-} \circ \varphi_{\mu} .
$$

Such isomorphisms induce algebra isomorphisms (denoted by the same name):

$$
T_{p}, T_{p}^{-}: \mathfrak{u}(\chi) \rightarrow \mathfrak{u}\left(s_{p}^{*} \chi\right) .
$$

Remark 2.10. If the homogeneous elements $X, Y \in \mathcal{U}_{p}^{+}(\chi)$ are such that $T_{p}(X), T_{p}(Y) \in$ $\mathcal{U}_{p}^{+}\left(s_{p}^{*} \chi\right), \operatorname{as} \operatorname{deg} T_{p}(X)=s_{p}(\operatorname{deg} X)$, it follows that

$$
T_{p}\left([X, Y]_{c}\right)=\left[T_{p}(X), T_{p}(y)\right]_{\underline{c}} .
$$

\section{An explicit PRESENTATiOn By GENERATORS AND RELAtions of Nichols ALGEBRAS OF DIAGONAL TYPE}

We shall obtain a family of isomorphisms induced by the ones in the previous Section. In this case we shall consider a quotient of $\mathcal{U}(\chi)$ by an ideal which is smaller than $\left(I^{-}(\chi)+\right.$ $\left.I^{+}(\chi)\right)$. Such ideal will be generated by some of the relations in Theorem 1.25, and will be the smallest one such that it is possible to define all the family of isomorphisms over the Weyl groupoid. It will give us a relation between the Hilbert series of these algebras, and new sets of roots. We shall use at the end the uniqueness of the root system, when the Weyl groupoid is finite.

We introduce some notation. We denote $\widetilde{q_{i j}}=q_{i j} q_{j i}$. Also,

$$
x_{i_{1} i_{2} \cdots i_{k}}=\left(\operatorname{ad}_{c} x_{i_{1}}\right) \cdots\left(\operatorname{ad}_{c} x_{i_{k-1}}\right) x_{i_{k}}, \quad i_{j} \in\{1, \ldots, \theta\} .
$$

For each $m \in \mathbb{N}$, we define the elements $x_{(m+1) \alpha_{i}+m \alpha_{j}} \in \mathcal{U}(\chi)$ recursively: 
- if $m=1, x_{2 \alpha_{i}+\alpha_{j}}:=\left(\operatorname{ad}_{c} x_{i}\right)^{2} x_{j}=x_{i i j}$,

- $x_{(m+2) \alpha_{i}+(m+1) \alpha_{j}}:=\left[x_{(m+1) \alpha_{i}+m \alpha_{j}}, x_{i j}\right]_{c}$.

We give now the main result of this section, which is Theorem 3.1 it gives an explicit presentation by generators and relations of any Nichols algebra of diagonal type with finite root system. We begin by proving several Lemmata to show the existence of Lusztig isomorphisms for some Hopf algebras. These Hopf algebras are intermediate between the tensor algebra and the Nichols algebras of a given braided vector space. Finally we use those Lusztig isomorphisms to prove the Theorem.

Theorem 3.1. Let $(V, c)$ be a finite-dimensional braided vector space of diagonal type, with braiding matrix $\left(q_{i j}\right)_{1 \leq i, j \leq \theta}, \theta=\operatorname{dim} V$, and fix a basis $x_{1}, \ldots, x_{\theta}$ of $V$ such that $c\left(x_{i} \otimes x_{j}\right)=q_{i j} x_{j} \otimes x_{i}$. Let $\chi$ be the bicharacter associated to $\left(q_{i j}\right)$. Assume that the root system $\Delta^{\chi}$ is finite. Then $\mathcal{B}(V)$ is presented by generators $x_{1}, \ldots, x_{\theta}$ and relations:

$$
\begin{array}{lr}
x_{\alpha}^{N_{\alpha}}, & \alpha \in \mathcal{O}(\chi) ; \\
\left(\operatorname{ad}_{c} x_{i}\right)^{m_{i j}+1} x_{j}, & q_{i i}^{m_{i j}+1} \neq 1 ; \\
x_{i}^{N_{i}}, & i \text { is not a Cartan vertex; }
\end{array}
$$

$\diamond$ if $i, j \in\{1, \ldots, \theta\}$ satisfy $q_{i i}=\widetilde{q_{i j}}=q_{j j}=-1$, and there exists $k \neq i, j$ such that ${\widetilde{q_{i k}}}^{2} \neq 1$ or ${\widetilde{q_{j k}}}^{2} \neq 1$,

$$
x_{i j}^{2}
$$

$\diamond$ if $i, j, k \in\{1, \ldots, \theta\}$ satisfy $q_{j j}=-1, \widetilde{q_{i k}}=\widetilde{q_{i j}} \widetilde{q_{k j}}=1, \widetilde{q_{i j}} \neq-1$,

$$
\left[x_{i j k}, x_{j}\right]_{c}
$$

$\diamond$ if $i, j \in\{1, \ldots, \theta\}$ satisfy $q_{j j}=-1, q_{i i} \widetilde{q_{i j}} \in \mathbb{G}_{6}, \widetilde{q_{i j}} \neq-1$, and also $q_{i i} \in \mathbb{G}_{3}$ or $m_{i j} \geq 3$,

$$
\left[x_{i i j}, x_{i j}\right]_{c} \text {; }
$$

$\diamond$ if $i, j, k \in\{1, \ldots, \theta\}$ satisfy $q_{i i}= \pm \widetilde{q_{i j}} \in \mathbb{G}_{3}, \widetilde{q_{i k}}=1$, and also $-q_{j j}=\widetilde{q_{i j}} \widetilde{q_{j k}}=1$ or $q_{j j}^{-1}=\widetilde{q_{i j}}=\widetilde{q_{j k}} \neq-1$,

$$
\left[x_{i i j k}, x_{i j}\right]_{c}
$$

$\diamond$ if $i, j, k \in\{1, \ldots, \theta\}$ satisfy $\widetilde{q_{i k}}, \widetilde{q_{i j}}, \widetilde{q_{j k}} \neq 1$,

$$
x_{i j k}-\frac{1-\widetilde{q_{j k}}}{q_{k j}\left(1-\widetilde{q_{i k}}\right)}\left[x_{i k}, x_{j}\right]_{c}-q_{i j}\left(1-\widetilde{q_{j k}}\right) x_{j} x_{i k} ;
$$

$\diamond$ if $i, j, k \in\{1, \ldots, \theta\}$ satisfy one of the following situations

$$
\begin{aligned}
& \circ q_{i i}=q_{j j}=-1,{\widetilde{q_{i j}}}^{2}=\widetilde{q_{j k}}-1, \widetilde{q_{i k}}=1 \text {, or } \\
& \circ \widetilde{q_{i j}}=q_{j j}=-1, q_{i i}=-{\widetilde{q_{j k}}}^{2} \in \mathbb{G}_{3}, \widetilde{q_{i k}}=1 \text {, or } \\
& \circ q_{k k}=\widetilde{q_{j k}}=q_{j j}=-1, q_{i i}=-\widetilde{q_{i j}} \in \mathbb{G}_{3}, \widetilde{q_{i k}}=1 \text {, or } \\
& \circ q_{j j}=-1, \widetilde{q_{i j}}=q_{i i}^{-2}, \widetilde{q_{j k}}=-q_{i i}^{-3}, \widetilde{q_{i k}}=1 \text {, or } \\
& \circ q_{i i}=q_{j j}=q_{k k}=-1, \pm \widetilde{q_{i j}}=\widetilde{q_{j k}} \in \mathbb{G}_{3}, \widetilde{q_{i k}}=1 \text {, }
\end{aligned}
$$

$$
\left[\left[x_{i j}, x_{i j k}\right]_{c}, x_{j}\right]_{c}
$$

$\diamond$ if $i, j, k \in\{1, \ldots, \theta\}$ satisfy $q_{i i}=q_{j j}=-1,{\widetilde{q_{i j}}}^{3}={\widetilde{q_{j k}}}^{-1}, \widetilde{q_{i k}}=1$,

$$
\left[\left[x_{i j},\left[x_{i j}, x_{i j k}\right]_{c}\right]_{c}, x_{j}\right]_{c}
$$

$\diamond$ if $i, j, k \in\{1, \ldots, \theta\}$ satisfy $q_{j j}={\widetilde{q_{i j}}}^{2}=\widetilde{q_{j k}} \in \mathbb{G}_{3}, \widetilde{q_{i k}}=1$,

$$
\left[\left[x_{i j k}, x_{j}\right]_{c} x_{j}\right]_{c}
$$


$\diamond$ if $i, j, k \in\{1, \ldots, \theta\}$ satisfy $q_{k k}=q_{j j}={\widetilde{q_{i j}}}^{-1}={\widetilde{q_{j k}}}^{-1} \in \mathbb{G}_{9}, \widetilde{q_{i k}}=1, q_{i i}=q_{k k}^{6}$

$$
\left[\left[x_{i i j}, x_{i i j k}\right]_{c}, x_{i j}\right]_{c}
$$

$\diamond$ if $i, j, k \in\{1, \ldots, \theta\}$ satisfy $q_{i i}={\widetilde{q_{i j}}}^{-1} \in \mathbb{G}_{9}, q_{j j}={\widetilde{q_{j k}}}^{-1}=q_{i i}^{5}, \widetilde{q_{i k}}=1, q_{k k}=q_{i i}^{6}$

$$
\left[\left[x_{i j k}, x_{j}\right]_{c}, x_{k}\right]_{c}-\left(1+\widetilde{q_{j k}}\right)^{-1} q_{j k}\left[\left[x_{i j k}, x_{k}\right]_{c}, x_{j}\right]_{c} ;
$$

$\diamond$ if $i, j, k \in\{1, \ldots, \theta\}$ satisfy $q_{j j}={\widetilde{q_{i j}}}^{3}=\widetilde{q_{j k}} \in \mathbb{G}_{4}, \widetilde{q_{i k}}=1$,

$$
\left.\left[\left[x_{i j k}, x_{j}\right]_{c}, x_{j}\right]_{c}, x_{j}\right]_{c}
$$

$\diamond$ if $i, j, k \in\{1, \ldots, \theta\}$ satisfy $q_{i i}=\widetilde{q_{i j}}=-1, q_{j j}={\widetilde{q_{j k}}}^{-1} \neq-1, \widetilde{q_{i k}}=1$,

$$
\left[x_{i j}, x_{i j k}\right]_{c}
$$

$\diamond$ if $i, j, k \in\{1, \ldots, \theta\}$ satisfy $q_{i i}=q_{k k}=-1, \widetilde{q_{i k}}=1, \widetilde{q_{i j}} \in \mathbb{G}_{3}, q_{j j}=-\widetilde{q_{j k}}= \pm \widetilde{q_{i j}}$,

$$
\left[x_{i}, x_{j j k}\right]_{c}-\left(1+q_{j j}^{2}\right) q_{k j}^{-1}\left[x_{i j k}, x_{j}\right]_{c}-\left(1+q_{j j}^{2}\right)\left(1+q_{j j}\right) q_{i j} x_{j} x_{i j k} ;
$$

$\diamond$ if $i, j, k, l \in\{1, \ldots, \theta\}$ satisfy $q_{j j} \widetilde{q_{i j}}=q_{j j} \widetilde{q_{j k}}=1, q_{k k}=-1, \widetilde{q_{i k}}=\widetilde{q_{i l}}=\widetilde{q_{j l}}=1$, ${\widetilde{q_{j k}}}^{2}={\widetilde{q_{l k}}}^{-1}=q_{l l}$,

$$
\left[\left[\left[x_{i j k l}, x_{k}\right]_{c}, x_{j}\right]_{c}, x_{k}\right]_{c} ;
$$

$\diamond$ if $i, j, k, l \in\{1, \ldots, \theta\}$ satisfy $\widetilde{q_{j k}}=\widetilde{q_{i j}}=q_{j j}^{-1} \in \mathbb{G}_{4}^{\prime} \cup \mathbb{G}_{6}^{\prime}, q_{i i}=q_{k k}=-1, \widetilde{q_{i k}}=\widetilde{q_{i l}}=$ $\widetilde{q_{j l}}=1, \widetilde{q_{j k}}=\widetilde{q_{l k}}$,

$$
\left[\left[x_{i j k},\left[x_{i j k l}, x_{k}\right]_{c}\right]_{c}, x_{j k}\right]_{c}
$$

$\diamond$ if $i, j, k, l \in\{1, \ldots, \theta\}$ satisfy $q_{l l}={\widetilde{q_{l k}}}^{-1}=q_{k k}={\widetilde{q_{j k}}}^{-1}=q^{2}, \widetilde{q_{i j}}=q_{i i}^{-1}=q^{3}$ for some $q \in \mathbf{k}^{\times}, q_{j j}=-1, \widetilde{q_{i k}}=\widetilde{q_{i l}}=\widetilde{q_{j l}}=1$,

$$
\left[\left[\left[x_{i j k}, x_{j}\right]_{c},\left[x_{i j k l}, x_{j}\right]_{c}\right]_{c}, x_{j k}\right]_{c}
$$

$\diamond$ if $i, j, k, l \in\{1, \ldots, \theta\}$ satisfy one of the following situations

$$
\begin{aligned}
& \circ q_{k k}=-1, q_{i i}={\widetilde{q_{i j}}}^{-1}=q_{j j}^{2}, \widetilde{q_{k l}}=q_{l l}^{-1}=q_{j j}^{3}, \widetilde{q_{j k}}=q_{j j}^{-1}, \widetilde{q_{i k}}=\widetilde{q_{i l}}=\widetilde{q_{j l}}=1, \text { or } \\
& \circ q_{i i}={\widetilde{q_{i j}}}^{-1}=-q_{l l}^{-1}=-\widetilde{q_{k l}}, q_{j j}=\widetilde{q_{j k}}=q_{k k}=-1, \widetilde{q_{i k}}=\widetilde{q_{i l}}=\widetilde{q_{j l}}=1,
\end{aligned}
$$

$$
\left[\left[x_{i j k l}, x_{j}\right]_{c}, x_{k}\right]_{c}-q_{j k}\left({\widetilde{q_{i j}}}^{-1}-q_{j j}\right)\left[\left[x_{i j k l}, x_{k}\right]_{c}, x_{j}\right]_{c} \text {; }
$$

$\diamond$ if $i, j, k \in\{1, \ldots, \theta\}$ satisfy $\widetilde{q_{j k}}=1, q_{i i}=\widetilde{q_{i j}}=-\widetilde{q_{i k}} \in \mathbb{G}_{3}$,

$$
\left[x_{i},\left[x_{i j}, x_{i k}\right]_{c}\right]_{c}+q_{j k} q_{i k} q_{j i}\left[x_{i i k}, x_{i j}\right]_{c}+q_{i j} x_{i j} x_{i i k}
$$

$\diamond$ if $i, j, k \in\{1, \ldots, \theta\}$ satisfy $q_{j j}=q_{k k}=\widetilde{q_{j k}}=-1, q_{i i}=-\widetilde{q_{i j}} \in \mathbb{G}_{3}, \widetilde{q_{i k}}=1$,

$$
\left[x_{i i j k}, x_{i j k}\right]_{c} \text {; }
$$

$\diamond$ if $i, j \in\{1, \ldots, \theta\}$ satisfy $-q_{i i},-q_{j j}, q_{i i} \widetilde{q_{i j}}, q_{j j} \widetilde{q_{i j}} \neq 1$,

$$
\left(1-\widetilde{q_{i j}}\right) q_{j j} q_{j i}\left[x_{i},\left[x_{i j}, x_{j}\right]_{c}\right]_{c}-\left(1+q_{j j}\right)\left(1-q_{j j} \widetilde{q_{i j}}\right) x_{i j}^{2} ;
$$

$\diamond$ if $i, j \in\{1, \ldots, \theta\}$ satisfy that $m_{i j} \in\{4,5\}$, or $q_{j j}=-1, m_{i j}=3, q_{i i} \in \mathbb{G}_{4}$,

$$
\left[x_{i}, x_{3 \alpha_{i}+2 \alpha_{j}}\right]_{c}-\frac{1-q_{i i} \widetilde{q_{i j}}-q_{i i}^{2} \widetilde{q_{i j}}{ }^{2} q_{j j}}{\left(1-q_{i i} \widetilde{q_{i j}}\right) q_{j i}} x_{i i j}^{2} ;
$$

$\diamond$ if $i, j \in\{1, \ldots, \theta\}$ satisfy $4 \alpha_{i}+3 \alpha_{j} \notin \Delta_{+}^{\chi}, q_{j j}=-1$ or $m_{j i} \geq 2$, and also $m_{i j} \geq 3$, or $m_{i j}=2, q_{i i} \in \mathbb{G}_{3}$,

$$
x_{4 \alpha_{i}+3 \alpha_{j}}=\left[x_{3 \alpha_{i}+2 \alpha_{j}}, x_{i j}\right]_{c} ;
$$

$\diamond$ if $i, j \in\{1, \ldots, \theta\}$ satisfy $3 \alpha_{i}+2 \alpha_{j} \in \Delta_{+}^{\chi}, 5 \alpha_{i}+3 \alpha_{j} \notin \Delta_{+}^{\chi}$, and $q_{i i}^{3} \widetilde{q_{i j}}, q_{i i}^{4} \widetilde{q_{i j}} \neq 1$,

$$
\left[x_{i i j}, x_{3 \alpha_{i}+2 \alpha_{j}}\right]_{c} \text {; }
$$


$\diamond$ if $i, j \in\{1, \ldots, \theta\}$ satisfy $4 \alpha_{i}+3 \alpha_{j} \in \Delta_{+}^{\chi}, 5 \alpha_{i}+4 \alpha_{j} \notin \Delta_{+}^{\chi}$,

$$
x_{5 \alpha_{i}+4 \alpha_{j}}=\left[x_{4 \alpha_{i}+3 \alpha_{j}}, x_{i j}\right]_{c} \text {; }
$$

$\diamond$ if $i, j \in\{1, \ldots, \theta\}$ satisfy $5 \alpha_{i}+2 \alpha_{j} \in \Delta_{+}^{\chi}, 7 \alpha_{i}+3 \alpha_{j} \notin \Delta_{+}^{\chi}$,

$$
\left[\left[x_{i i i j}, x_{i i j}\right], x_{i i j}\right]_{c} \text {; }
$$

$\diamond$ if $i, j \in\{1, \ldots, \theta\}$ satisfy $q_{j j}=-1,5 \alpha_{i}+4 \alpha_{j} \in \Delta_{+}^{\chi}$,

$$
\left[x_{i i j}, x_{4 \alpha_{i}+3 \alpha_{j}}\right]_{c}-\frac{b-\left(1+q_{i i}\right)\left(1-q_{i i} \zeta\right)\left(1+\zeta+q_{i i} \zeta^{2}\right) q_{i i}^{6} \zeta^{4}}{a q_{i i}^{3} q_{i j}^{2} q_{j i}^{3}} x_{3 \alpha_{i}+2 \alpha_{j}}^{2},
$$

where $\zeta=\widetilde{q_{i j}}, a=(1-\zeta)\left(1-q_{i i}^{4} \zeta^{3}\right)-\left(1-q_{i i} \zeta\right)\left(1+q_{i i}\right) q_{i i} \zeta, b=(1-\zeta)\left(1-q_{i i}^{6} \zeta^{5}\right)-a q_{i i} \zeta$.

In what follows we will use implicitly the isomorphism $\mathcal{B}(V) \cong \mathfrak{u}^{+}(\chi)$ determined by $x_{i} \mapsto E_{i}$; in this way, we identify $\mathcal{B}(V)$ as a subalgebra of $\mathfrak{u}(\chi)$.

For any bicharacter $\chi$ whose root system is finite, $\mathcal{J}^{+}(\chi)$ denotes the ideal of $\mathcal{U}^{+}(\chi)$ generated by all the relations in Theorem 3.1, except (40), plus the quantum Serre relations $\left(\operatorname{ad}_{c} x_{i}\right)^{1-a_{i j}} x_{j}$ for those vertices such that $q_{i i}^{a_{i j}}=q_{i j} q_{j i}=q_{i i}$. The last ingredient is to obtain a quotient of all the algebras $\mathcal{U}_{p}(\chi), 1 \leq p \leq \theta$.

Call also $\mathcal{J}^{-}(\chi):=\phi_{4}\left(\mathcal{J}^{+}(\chi)\right), \mathcal{J}(\chi):=\left(\mathcal{J}^{+}(\chi)+\mathcal{J}^{-}(\chi)\right)$,

$$
U(\chi):=\mathcal{U}(\chi) / \mathcal{J}(\chi), \quad U^{ \pm}(\chi):=\mathcal{U}^{ \pm}(\chi) / \mathcal{J}^{ \pm}(\chi) .
$$

We prove first that $\mathcal{J}^{+}(\chi)$ is contained in the ideal defining the corresponding Nichols algebra. The following Lemma was proved with Agustín García Iglesias and is implicit in other papers.

Lemma 3.2. Let $I \subset T(V)$ be a braided homogeneous biideal of $T(V)$, so there exists a surjective morphism of braided graded Hopf algebras $\pi: R:=T(V) / I \rightarrow \mathcal{B}(V)$. Let $\mathbf{x} \in \operatorname{ker} \pi, \mathbf{x} \neq 0$ of minimal degree $k \geq 2$. Then $\mathbf{x}$ is primitive.

Proof. As $\pi$ is a morphism of graded braided bialgebras, $\operatorname{ker} \pi$ is a graded biideal:

$$
\Delta(\mathbf{x})=\mathbf{x} \otimes 1+1 \otimes \mathbf{x}+\sum_{j=1}^{n} b_{j} \otimes c_{j} \in \operatorname{ker} \pi \otimes R+R \otimes \operatorname{ker} \pi,
$$

for some homogeneous elements $b_{j}, c_{j} \in \bigoplus_{i=1}^{k-1} R_{i}$, such that $\operatorname{deg}\left(b_{j}\right)+\operatorname{deg}\left(c_{j}\right)=k$. For each $j$ we may assume either $b_{j} \in \operatorname{ker} \pi$ or $c_{j} \in \operatorname{ker} \pi$. If $b_{j} \in \operatorname{ker} \pi$, then $b_{j}=0$ by the minimality condition on $k$. Similarly, if $c_{j} \in \operatorname{ker} \pi$, then $c_{j}=0$. Hence $\mathbf{x}$ is primitive in $R$.

We will work with $\mathbb{N}_{0}^{\theta}$-graded ideals, so the following notation will be useful: given $\beta=\sum_{i} b_{i} \alpha_{i}, \gamma=\sum_{i} c_{i} \alpha_{i}$, for some $b_{i}, c_{i} \in \mathbb{N}_{0}$, we say that $\beta \geq \gamma$ (respectively, $\beta>\gamma$ ) if $b_{i} \geq c_{i}$ (respectively, $b_{i}>c_{i}$ ) for all $i \in\{1, \ldots, \theta\}$.

Proposition 3.3. $\mathcal{J}^{+}(\chi)$ is a braided biideal of $\mathcal{U}^{+}(\chi)$, and there exist a canonical projection of Hopf algebras $\pi_{\chi}: U(\chi) \rightarrow \mathfrak{u}(\chi)$ such that $\pi\left(U^{ \pm}(\chi)\right)=\mathfrak{u}^{ \pm}(\chi)$.

Moreover, the multiplication $m: U^{+}(\chi) \otimes U^{0}(\chi) \otimes U^{-}(\chi) \rightarrow U(\chi)$ is an isomorphism of graded vector spaces.

Proof. We can order the relations according to their $\mathbb{N}$-graduation. When we quotient by the relations of degree at most $n-1$, the relations of degree $n$ are primitive by Lemma 3.2, because for any of them we can see that the relations in Theorem 1.25 of degree at most $n-1$ are verified. Moreover, for a relation of degree $\alpha \in \mathbb{N}_{0}^{\theta}$, it is enough to verify that the relations of $\mathbb{N}^{\theta}$-degree lower than $\alpha$ hold in this partial quotient. For example, 
each quantum Serre relation is primitive, and the same holds for $x_{i}^{N_{i}}$; therefore, when we quotient by these relation we have that $\mathbf{x}=\left[\left(\operatorname{ad}_{c} x_{i}\right)^{2} x_{j},\left(\operatorname{ad}_{c} x_{i}\right) x_{j}\right]_{c}$ is primitive under the conditions for (45), because we have quotiented by $x_{i}^{3}, x_{j}^{2}$, so it also holds that

$$
\left(\operatorname{ad}_{c} x_{i}\right)^{3} x_{j}=\left(\operatorname{ad}_{c} x_{j}\right)^{2} x_{i}=0 .
$$

We work in a similar way with the other relations so each partial quotient is a braided bialgebra (and then a Hopf algebra with the induced antipode); finally, $U^{+}(\chi)$ is a braided bialgebra, because $\mathcal{J}^{+}(\chi)$ is a braided biideal.

By the definition of Nichols algebra we conclude that $\mathcal{J}^{+}(\chi) \subseteq I^{+}(\chi)$. By Lemma 2.5, $\mathcal{J}^{+}(\chi) \mathcal{U}^{0}(\chi) \mathcal{U}^{-}(\chi)$ is a Hopf ideal of $\mathcal{U}(\chi)$, and then the equivalent conditions in Proposition 2.4 hold. Therefore there exists a projection of Hopf algebras and a triangular decomposition as in the Proposition.

Now we prove that the isomorphisms at the beginning of Theorem 2.9 induce isomorphisms between the corresponding algebras $U(\chi)$. The first step is to prove that $T_{p}(\mathcal{J}(\chi)) \subset \mathcal{J}\left(s_{p}^{*} \chi\right)$, which will be proved considering each relation generating the ideal. The following two Lemmata help us to reduce the number of explicit computations.

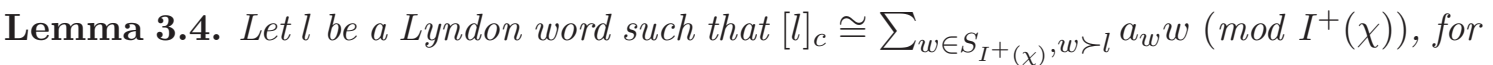
some $a_{w} \in \mathbf{k}$. Let I be a braided Hopf ideal $\mathbb{N}^{\theta}$-graded of $\mathcal{U}^{+}(\chi)$ such that the set of good words $S_{I^{+}(\chi)}, S_{I}$ coincide for those terms $w \succ l$, and assume that $l$ is written as a linear combination of words greater than $l$ modulo $I$. Then $[l]_{c} \cong \sum_{w \in S_{I^{+}(\chi)}, w \succ l} a_{w} w(\bmod I)$.

Proof. It is a direct consequence of Corollary 1.14 by this result, $[l]_{c}$ is written as a linear combination of good hyperwords greater than $[l]_{c}$ modulo $I$. Such hyperwords coincide with the corresponding good hyperwords for $I^{+}(\chi)$ by hypothesis, and also $I \subseteq I^{+}(\chi)$. Hence the linear combination should be the same, because the good hyperwords generate a linear complement of the ideal in $\mathcal{U}^{+}(\chi)$.

Lemma 3.5. Let $I$ be an $\mathbb{N}_{0}^{\theta}$-homogeneous ideal of $T(V), \theta=\operatorname{dim} V$. Let $S, T$ be two minimal sets of homogeneous generators of $I$. Assume that for each $\alpha \in \mathbb{N}^{\theta}$ there exists at most one generator in $S$ (respectively in $T$ ) of degree $\alpha$, and denote by $I(S, \alpha)$ (respectively, $I(T, \alpha)$ ) the ideal generated by the elements of $S$ (respectively, $T$ ) of degree $\beta<\alpha$.

For each $s \in S$ of degree $\alpha \in \mathbb{N}_{0}^{\theta}$, there exists $t \in T$ of the same degree, and $c \in \mathbf{k}^{\times}$ such that $s \cong c t(\bmod (I(S, \alpha))$, and then $I(S, \alpha)=I(T, \alpha)$.

Proof. We prove it by induction on the degree of the generators. Let $s$ be of degree $\alpha$, minimal for the partial order defined on $\mathbb{N}_{0}^{\theta}$. Therefore $\operatorname{dim} I_{\alpha}=1$, so there exists an element of $T$ which belongs to this subspace of $I$ of dimension 1.

If the degree of $s$ is not minimal, we apply inductive hypothesis for all the generators in lower degree, so for each $s^{\prime}$ of lower degree there exists a corresponding $t^{\prime} \in T$ of the same degree which satisfies the conditions above, and $I(S, \alpha)=I(T, \alpha)$. Therefore

$$
\operatorname{dim} I(S, \alpha)_{\alpha}=\operatorname{dim} I(T, \alpha)_{\alpha}=\operatorname{dim} I_{\alpha}-1,
$$

because $S$ is a minimal set of generators, and by hypothesis there exists a unique generator of degree $\alpha$. As $T$ is also a set of generators of $I$, there exists $t \in I-I(T, \alpha)=I-I(S, \alpha)$, of degree $\alpha$, so the statement follows.

Remark 3.6. This Lemma lets us to identify relations of the same degree for two sets of minimal generators of an ideal, up to relations of lower degree and scalars. In this way we can consider relations from Theorem 1.25 for a fixed order on the letters, and consider relations for another order. If we have a minimal set and this set contains relations all in 
different degrees (as we will have for the set of relations of the Nichols algebra or some partial quotients), then we can find a correspondence as above between the relations of the same $\mathbb{Z}^{\theta}$-degree.

For example, if $q_{i i}^{m_{i j}+1} \neq 1$ for some pair of vertices $i, j$, then the quantum Serre relation $\left(\operatorname{ad}_{c} x_{i}\right)^{m_{i j}+1} x_{j}$ is a generator for the minimal set of generators corresponding to the order $x_{i}<x_{j}$, so for the order $x_{i}>x_{j}$ we have:

$$
\left[x_{j} x_{i}^{m_{i j}+1}\right]_{c}=\left[\cdots\left[\left[\left(\operatorname{ad}_{c} x_{j}\right) x_{i}, x_{i}\right]_{c}, \cdots,\right]_{c}, x_{i}\right]_{c}=a\left(\operatorname{ad}_{c} x_{i}\right)^{m_{i j}+1} x_{j},
$$

for some scalar $a \in \mathbf{k}^{\times}$.

Also, if $q_{i i} \in \mathbb{G}_{3}, \widetilde{q_{i j}} \in\left\{ \pm q_{i i},-1\right\}, q_{j j}=-1$, we notice that

$$
\left[\left(\operatorname{ad}_{c} x_{i}\right)^{2} x_{j},\left(\operatorname{ad}_{c} x_{i}\right) x_{j}\right]_{c} \cong b\left[\left(\operatorname{ad}_{c} x_{j}\right) x_{i},\left[\left(\operatorname{ad}_{c} x_{j}\right) x_{i}, x_{i}\right]_{c}\right]_{c}(\bmod I),
$$

for some $b \in \mathbf{k}^{\times}$, where $I$ is the ideal generated by $x_{i}^{3}$ and $x_{j}^{2}$, because such relations correspond to different minimal sets of generators of the ideal of relations of the Nichols algebra, and these are the generators of degree $3 \alpha_{i}+2 \alpha_{j}$ for each set.

Lemma 3.7. Let I be a $\mathbb{Z}^{\theta}$-graded ideal of $\mathcal{U}_{p}^{+}(\chi)$. Let $Y, Z \in \mathcal{U}_{p}^{+}(\chi) / I$ be homogeneous elements of degree $\beta, \gamma \in \mathbb{N}_{0}^{\theta}$, respectively, such that $\left(\operatorname{ad}_{c} E_{p}\right) Y=0$. Then,

$$
\left[\left(\operatorname{ad}_{c} E_{p}\right) Z, Y\right]_{c}=\left(\operatorname{ad}_{c} E_{p}\right)[Z, Y]_{c} .
$$

If also $\chi\left(\alpha_{p}, \beta\right) \chi\left(\beta, \alpha_{p}\right)=1$, then

$$
\chi\left(\alpha_{p}, \beta\right)\left[Y,\left(\operatorname{ad}_{c} E_{p}\right) Z\right]_{c}=\left(\operatorname{ad}_{c} E_{p}\right)[Y, Z]_{c} .
$$

Proof. Both identities follow from (91). For example, for the second one,

$$
\begin{aligned}
\left(\operatorname{ad}_{c} E_{p}\right)[Y, Z]_{c} & =\left[E_{p},[Y, Z]_{c}\right]_{c}=\left[\left[E_{p}, Y\right]_{c}, Z\right]_{c}+\chi\left(\alpha_{p}, \beta\right) Y\left[E_{p}, Z\right]_{c}-\chi(\beta, \gamma)\left[E_{p}, Z\right]_{c} Y \\
& =\chi\left(\alpha_{p}, \beta\right)\left(Y\left(\operatorname{ad}_{c} E_{p}\right) Z-\chi(\beta, \gamma) \chi\left(\beta, \alpha_{p}\right)\left(\operatorname{ad}_{c} E_{p}\right) Z Y\right) \\
& =\chi\left(\alpha_{p}, \beta\right)\left[Y,\left(\operatorname{ad}_{c} E_{p}\right) Z\right]_{c},
\end{aligned}
$$

where we use the condition $\chi\left(\alpha_{p}, \beta\right) \chi\left(\beta, \alpha_{p}\right)=1$.

Lemma 3.8. Let $i, p \in\{1, \ldots, \theta\}$ be such that $m_{p i} \geq 2$ and $m_{i p}=1$. Then, in $U\left(s_{p}^{*} \chi\right)$,

$$
\left[\underline{E}_{i, m_{p i}}^{+}, \underline{E}_{i, m_{p i}-1}^{+}\right]_{\underline{c}}=\left[\left(\operatorname{ad}_{\underline{c}} \underline{E}_{p}\right)^{m_{p i}} \underline{E}_{i},\left(\operatorname{ad}_{\underline{c}} \underline{E}_{p}\right)^{m_{p i}-1} \underline{E}_{i}\right]_{\underline{c}}=0
$$

Remark 3.9. Such relation belongs to the ideal $I^{+}\left(s_{p}^{*} \chi\right)$. In fact, as $2 \alpha_{i}+\alpha_{p} \notin \Delta_{+}^{\chi}$, we have $s_{p}\left(2 \alpha_{i}+\alpha_{p}\right)=2 \alpha_{i}+\left(2 m_{p i}-1\right) \alpha_{p} \notin \Delta_{+}^{\chi}$, so such relation holds in the corresponding Nichols algebra $\mathfrak{u}^{+}\left(s_{p}^{*} \chi\right)$.

On the other hand, some of these relations are generators of the ideal $\mathcal{J}\left(s_{p}^{*} \chi\right)$ by definition, for example (45). We prove here that the other relations not in the definition of this ideal are redundant; that is, they are generated by relations of lower degree.

Proof. We consider the different possible values of $m_{p i}$; we begin with $m_{p i}=2$. Therefore $q_{p p} \in \mathbb{G}_{3}$ or $q_{p p}^{2} q_{i p} q_{p i}=1$, and also $q_{i i}=-1$ or $q_{i i} q_{i p} q_{p i}=1$. If $\underline{m}_{i p}=1$ for $s_{p}^{*} \chi$, then $p, i$ determine a subdiagram of standard type. If $q_{p p}^{3} \neq 1$ or $q_{i i} \neq-1$ then $E_{p}^{2} E_{i} E_{p} E_{i}$ is written as a linear combination of words greater than $E_{p}^{2} E_{i} E_{p} E_{i}$, modulo $\mathcal{J}\left(s_{p}^{*} \chi\right)$, using the quantum Serre relations, because in the first case $E_{p}^{2} E_{i} E_{p}$ appears with non-zero coefficient in $\left(\operatorname{ad}_{c} E_{p}\right)^{3} E_{i}$, so $E_{p}^{2} E_{i} E_{p} E_{i}$ is a linear combination of greater words and $E_{p}^{3} E_{i}^{2}$, but this last word is in the ideal if $q_{i i}=-1$, or $E_{p} E_{i}^{2}$ appears in $\left(\operatorname{ad}_{c} E_{i}\right)^{2} E_{p}$ with nonzero coefficient, so in both cases we obtain $E_{p}^{2} E_{i} E_{p} E_{i}$ as a combination of greater words. 
Using Lemma [3.4, we conclude that $\left[\underline{E}_{i, 2}^{+}, \underline{E}_{i, 1}^{+}\right]_{c}=0$. A similar proof in the case $q_{p p}^{3}=1$, $q_{i i} \neq-1$ gives us the same conclusion. If $q_{p p}^{3}=1, q_{i i}=-1$, the relation corresponds to (45), which is by definition a generator of $\mathcal{J}\left(s_{p}^{*} \chi\right)$.

If $m_{p i}=2$ and $\underline{m}_{i p}>1$ for $s_{p}^{*} \chi$, then (62) is a generator of $\mathcal{J}\left(s_{p}^{*} \chi\right)$, and then $E_{p} E_{i} E_{p} E_{i}$ is a linear combination of $E_{p}^{2} E_{i}^{2}$ and greater words. Therefore $\left[\underline{E}_{i, 2}^{+}, \underline{E}_{i, 1}^{+}\right]_{c} \in \mathcal{J}\left(s_{p}^{*} \chi\right)$, by a similar argument.

If $m_{p i}=3$, then $\underline{m}_{i p}=1$ for $s_{p}^{*} \chi$, or there exists $\zeta \in \mathbb{G}_{24}$ such that $q_{p p}=\zeta^{6}, q_{i i}^{-1}=$ $q_{p i} q_{i p}=\zeta$. For the first case, we notice that (63) holds also if $q_{p p} \notin \mathbb{G}_{4}$, because $\underline{E}_{p}^{3} \underline{E}_{i} \underline{E}_{p}$ can be written as a linear combination of other words from the quantum Serre relation $\left(\operatorname{ad}_{\underline{c}} \underline{E}_{p}\right)^{4} \underline{E}_{i}=0$, and then $\underline{E}_{p}^{3} \underline{E}_{i} \underline{E}_{p} \underline{E}_{i}$ is a linear combination of greater words multiplying by $\underline{E}_{i}$, so we apply Lemma 3.4 , from this relation we work as above, so we write $E_{p}^{2} E_{i} E_{p}^{2} E_{i}$ as a linear combination of other words and deduce that $E_{p}^{3} E_{i} E_{p}^{2} E_{i}$ is a linear combination of greater words, and we can apply Lemma 3.4 again. For the second case, we write $\underline{E}_{p}^{3} \underline{E}_{i} \underline{E}_{p}^{2} \underline{E}_{i}$ as a linear combination of greater words using the quantum Serre relations or the relation (63), with the same conclusion.

If $m_{p i}=4,5$, then $\underline{m}_{i p}=1$ for $s_{p}^{*} \chi$. Therefore we work as before and we obtain the desired relation from (63) or (45), according to $3 \alpha_{p}+2 \alpha_{i}$ belongs to $\Delta_{+}^{s_{p}^{*} \chi}$ or not. In both situations, we can write $\underline{E}_{p}^{4} \underline{E}_{i} \underline{E}_{p}^{3} \underline{E}_{i}$ or $\underline{E}_{p}^{5} \underline{E}_{i} \underline{E}_{p}^{4} \underline{E}_{i}$ as a linear combination of greater words, so we apply Lemma 3.4 again.

We will prove now that $T_{p}(x) \in \mathcal{J}\left(s_{p}^{*} \chi\right)$ for any generator $x$ of the ideal $\mathcal{J}^{+}(\chi)$ so we will have a family of morphisms between the algebras $U(\chi)$.

Lemma 3.10. Let $i$ be a non-Cartan vertex. Then $T_{p}\left(E_{i}^{N_{i}}\right) \in \mathcal{J}\left(s_{p}^{*} \chi\right)$.

If $i, j$ satisfy $q_{i i}=\widetilde{q_{i j}}=q_{j j}=-1$, and there exists $k$ such that $\widetilde{q_{i k}} \neq-1$ or ${\widetilde{q_{j k}}}^{2} \neq-1$, then $T_{p}\left(E_{i j}^{2}\right) \in \mathcal{J}\left(s_{p}^{*} \chi\right)$.

Proof. Consider the first relation. If $i=p$, then $p$ is not Cartan for $\chi$, so $p$ is not Cartan for $s_{p}^{*} \chi$ too. Therefore, by the definition of the ideal $\mathcal{J}\left(s_{p}^{*} \chi\right)$,

$$
T_{p}\left(E_{p}^{N_{p}}\right)=\underline{F}_{p}^{N_{p}}=\phi_{4}\left(\underline{E}_{p}^{N_{p}}\right) \in \mathcal{J}\left(s_{p}^{*} \chi\right) .
$$

We consider then $i \neq p$. In such case, $T_{p}\left(E_{i}^{N_{i}}\right)=\left(\underline{E}_{i, m_{p i}}^{+}\right)^{N_{i}}$.

If $m_{p i}=0$, then $\underline{E}_{i, 0}^{+}=\underline{E}_{i}$ and $q_{i p} q_{p i}=1$, so for each $j \neq p$ we have $\underline{q}_{i j} \underline{q}_{j i}=\widetilde{q_{i j}}$. Therefore $i$ is not Cartan for $s_{p}^{*} \chi$, and $T_{p}\left(E_{i}^{N_{i}}\right)=\underline{E}_{i}^{N_{i}} \in \mathcal{J}\left(s_{p}^{*} \chi\right)$.

Consider $m_{p i} \neq 0$. As $i$ is not Cartan, there exists $j \neq i$ such that $q_{i i}^{m_{i j}} \widetilde{q_{i j}} \neq 1$.

Assume first that $m_{i p}+1=N_{i}$. If $m_{i p}=1$, that is $q_{i i}=-1$, there are two possibilities. If $q_{i p} q_{p i} \neq-1$, using Lemma 3.8, the identity (9) and the quantum Serre relation $\left(\operatorname{ad}_{\underline{c}} \underline{E}_{p}\right)^{m_{p i}+1} \underline{E}_{i}=0$, we compute in $U\left(s_{p}^{*} \chi\right)$,

$$
\begin{aligned}
0 & =\left[\underline{E}_{p},\left[\underline{E}_{i, m_{p i}}^{+}, \underline{E}_{i, m_{p i}-1}^{+}\right]_{c}\right]_{\underline{c}} \\
& =\left(s_{p}^{*} \chi\left(\alpha_{p}, m_{p i} \alpha_{p}+\alpha_{i}\right)-s_{p}^{*} \chi\left(m_{p i} \alpha_{p}+\alpha_{i},\left(m_{p i}-1\right) \alpha_{p}+\alpha_{i}\right)\right)\left(\underline{E}_{i, m_{p i}}^{+}\right)^{2} \\
& =\left(\chi\left(-\alpha_{p}, \alpha_{i}\right)-\chi\left(\alpha_{i}, \alpha_{p}+\alpha_{i}\right)\right)\left(\underline{E}_{i, m_{p i}}^{+}\right)^{2}=q_{p i}^{-1}\left(1+q_{i p} q_{p i}\right)\left(\underline{E}_{i, m_{p i}}^{+}\right)^{2},
\end{aligned}
$$

so $T_{p}\left(E_{i}^{2}\right)=\left(\underline{E}_{i, m_{p i}}^{+}\right)^{2} \in \mathcal{J}\left(s_{p}^{*} \chi\right)$. If $q_{p i} q_{i p}=-1$, there are 3 possible subdiagrams determined by $i, p$ : it is standard with $q=-1$, or it is Cartan of type $B_{2}$ with $q \in \mathbb{G}_{4}$, 
or it is Cartan of type $G_{2}$ with $q \in G_{6}$. For the first case, if the diagram is of type $A_{2}$ associated to $q=-1$, it follows by definition of the ideal $\mathcal{J}\left(s_{p}^{*} \chi\right)$; for the other cases, we write $\underline{E}_{p}^{m_{p i}} \underline{E}_{i} \underline{E}_{p}^{m_{p i}} \underline{E}_{i}$ as a linear combination of greater words using (45) or the quantum Serre relations, and also the previous Lemmata.

If $m_{i p}>1$ and $m_{p i}=1$, we compute, using (9) and the relation $\left(\operatorname{ad}_{\underline{c}} \underline{E}_{p}\right)^{2} \underline{E}_{i}=0$,

$$
\begin{aligned}
\operatorname{ad}_{\underline{c}} \underline{E}_{p}\left[\underline{E}_{i, 1}^{+}, \underline{E}_{i}\right]_{\underline{c}} & =\left(s_{p}^{*} \chi\left(\alpha_{p}, \alpha_{i}+\alpha_{p}\right)-s_{p}^{*} \chi\left(\alpha_{i}+\alpha_{p}, \alpha_{i}\right)\right)\left(\underline{E}_{i, 1}^{+}\right)^{2} \\
& =q_{p i}^{-1}\left(1-q_{i i} q_{i p} q_{p i}\right)\left(\underline{E}_{i, 1}^{+}\right)^{2} .
\end{aligned}
$$

From this relation and (9) again, we calculate

$$
\begin{aligned}
\operatorname{ad}_{\underline{c}} \underline{E}_{p}\left[\underline{E}_{i, 1}^{+},\left[\underline{E}_{i, 1}^{+}, \underline{E}_{i}\right]_{\left.\underline{c}]_{\underline{c}}\right]_{\underline{p}}}\right. & \left(s_{p}^{*} \chi\left(\alpha_{p}, \alpha_{i}+\alpha_{p}\right)-s_{p}^{*} \chi\left(\alpha_{i}+\alpha_{p}, 2 \alpha_{i}+\alpha_{p}\right)\right) \\
& \left(q_{p i}^{-1}-q_{i i} q_{i p}\right)\left(\underline{E}_{i, 1}^{+}\right)^{3} \\
= & q_{p i}^{-2}\left(1-q_{i i} q_{i p} q_{p i}\right)\left(1-q_{i i}^{2} q_{i p} q_{p i}\right)\left(\underline{E}_{i, 1}^{+}\right)^{3} .
\end{aligned}
$$

So if $m_{i p}=2$ and $m_{p i}=1$, it follows that $\alpha_{p}+3 \alpha_{i} \notin \Delta_{+}^{\chi}$, and

$$
s_{p}\left(\alpha_{p}+3 \alpha_{i}\right)=3 \alpha_{i}+2 \alpha_{p} \notin \Delta_{+}^{s_{p}^{*} \chi} .
$$

Using the previous Lemma, $\left[\left(\operatorname{ad}_{\underline{c}} \underline{E}_{i}\right)^{2} \underline{E}_{p},\left(\operatorname{ad}_{\underline{c}} \underline{E}_{i}\right) \underline{E}_{p}\right]_{\underline{c}} \in \mathcal{J}^{+}\left(s_{p}^{*} \chi\right)$, so

$$
\left[\underline{E}_{i, 1}^{+},\left[\underline{E}_{i, 1}^{+}, \underline{E}_{i}\right]_{\underline{c}}\right]_{\underline{c}} \in \mathcal{J}\left(s_{p}^{*} \chi\right)
$$

because we apply Lemma 3.5 if the relation belongs to a minimal set of generators $\left(q_{i i}^{2} q_{i p} q_{p i} \neq 1\right.$, so $\left.q_{i i} \in \mathbb{G}_{3}\right)$, or we compute it directly for the cases in which it is Cartan of type $B_{2}$ or standard with $q_{p p}=-1$. Then, by a similar argument,

$$
T_{p}\left(E_{i}^{3}\right)=\left(\underline{E}_{i, 1}^{+}\right)^{3} \in \mathcal{J}\left(s_{p}^{*} \chi\right) .
$$

If $m_{i p}=3, m_{p i}=1$, we have that $s_{p}\left(\alpha_{p}+4 \alpha_{i}\right)=4 \alpha_{i}+3 \alpha_{p} \notin \Delta_{+}^{s_{p}^{*} \chi}$, so

$$
\left[\underline{E}_{i, 1}^{+},\left[\underline{E}_{i, 1}^{+},\left[\underline{E}_{i, 1}^{+}, \underline{E}_{i}\right]_{\underline{c}}\right]_{\underline{c}}\right]_{\underline{c}} \in \mathcal{J}\left(s_{p}^{*} \chi\right),
$$

by a similar argument, using (64). In this case we deduce that $\left(\underline{E}_{i, 1}^{+}\right)^{4} \in \mathcal{J}\left(s_{p}^{*} \chi\right)$.

If $m_{i p}=4$, then $q_{i i}^{4} q_{i p} q_{p i} \neq 1$, so $\left(\underline{E}_{i, 1}^{+}\right)^{5} \in \mathcal{J}\left(s_{p}^{*} \chi\right)$ in a similar way, using (6ㅜ). We notice that there are no diagrams such that $q_{i i}^{m_{i p}+1}=1$ and $m_{i p} \geq 5$.

Now we consider $m_{i p}, m_{p i}>1$, so there are 3 possibilities:

- $m_{i p}=m_{p i}=2$, so (64) is a generator of $\mathcal{J}\left(s_{p}^{*} \chi\right)$, and $q_{p p} \in \mathbb{G}_{3}$. Therefore we write $\underline{E}_{i} \underline{E}_{p}^{2} \underline{E}_{i} \underline{E}_{p}^{2} \underline{E}_{i}$ as a linear combination of other words, which begin with $\underline{E}_{p}$ or they contain $\underline{E}_{p}^{3}$ as a factor. If we multiply by $\underline{E}_{p}^{2}$ on the left, $\underline{E}_{p}^{2} \underline{E}_{i} \underline{E}_{p}^{2} \underline{E}_{i} \underline{E}_{p}^{2} \underline{E}_{i}$ is a linear combination of greater words modulo $\mathcal{J}\left(s_{p}^{*} \chi\right)$, because $\underline{E}_{p}^{3} \in \mathcal{J}\left(s_{p}^{*} \chi\right)$, so $T_{p}\left(E_{i}^{3}\right)=\left(\left(\operatorname{ad}_{c} \underline{E}_{p}\right)^{2} \underline{E}_{i}\right)^{3} \in \mathcal{J}\left(s_{p}^{*} \chi\right)$. 
- $m_{i p}=3, m_{p i}=2$, so $q_{i i}=\zeta^{6}, q_{p p}=\zeta^{8}, q_{i p} q_{p i}=\zeta^{11}$ for some $\zeta \in \mathbb{G}_{24}$, and (68) is a generator of the ideal $\mathcal{J}\left(s_{p}^{*} \chi\right)$. Using this relation we write $\underline{E}_{i} \underline{E}_{p}^{2} \underline{E}_{i} \underline{E}_{p}^{2} \underline{E}_{i} \underline{E}_{p}^{2} \underline{E}_{i}$ as a linear combination of words beginning with $\underline{E}_{p}$ or words containing $\underline{E}_{p}^{3}$ as a factor. Multiplying by $\underline{E}_{p}^{2}$ on the left, we write $\left(\underline{E}_{p}^{2} \underline{E}_{i}\right)^{4}$ as a linear combination of greater words modulo $\mathcal{J}\left(s_{p}^{*} \chi\right)$, because $\underline{E}_{p}^{3} \in \mathcal{J}\left(s_{p}^{*} \chi\right)$, so $T_{p}\left(E_{i}^{4}\right)=$ $\left(\left(\operatorname{ad}_{c} \underline{E}_{p}\right)^{2} \underline{E}_{i}\right)^{4} \in \mathcal{J}\left(s_{p}^{*} \chi\right)$.

- $m_{i p}=2, m_{p i}=3$, so there are two possible diagrams; in both cases (67) is a generator of $\mathcal{J}\left(s_{p}^{*} \chi\right)$. From this relation we write $\underline{E}_{i} \underline{E}_{p}^{3} \underline{E}_{i} \underline{E}_{p}^{3} \underline{E}_{i}$ as a combination of words beginning with $\underline{E}_{p}$ o containing $\underline{E}_{p}^{4}$. Multiplying on the left by $\underline{E}_{p}^{3}$, $\underline{E}_{p}^{3} \underline{E}_{i} \underline{E}_{p}^{3} \underline{E}_{i} \underline{E}_{p}^{3} \underline{E}_{i}$ can be written as a linear combination of greater words, modulo $\mathcal{J}\left(s_{p}^{*} \chi\right)$, because $\underline{E}_{p}^{4} \in \mathcal{J}\left(s_{p}^{*} \chi\right)$ or $\left(\operatorname{ad}_{\underline{c}} \underline{E}_{p}\right)^{4} \underline{E}_{i} \in \mathcal{J}\left(s_{p}^{*} \chi\right)$, so, in both cases, $T_{p}\left(E_{i}^{3}\right)=\left(\left(\operatorname{ad}_{c} \underline{E}_{p}\right)^{3} \underline{E}_{i}\right)^{3} \in \mathcal{J}\left(s_{p}^{*} \chi\right)$.

Finally we consider $q_{i i}^{m_{i p}} q_{i p} q_{p i}=1, m_{i p}<N_{i}-1$, so there exists $j \neq p$ such that $1 \leq m_{i p}<m_{i j}=N_{i}-1$. In this case, $i, j, p$ determine a connected diagram, where $i$ is not Cartan, connected with $j$ and $p$, and also $q_{i i}$ is a root of unity of order $N_{i}>2$. We have the following possible diagrams under the previous conditions:

- $q_{i i} \in \mathbb{G}_{3}, q_{p p} \in\left\{q_{i i},-1\right\}, m_{i p}=m_{p i}=1, m_{i j}=2, m_{p j}=m_{j p}=0$, or

- $q_{i i} \in \mathbb{G}_{4}, q_{p p}=-1, q_{i p} q_{p i} q_{i i}=1, \widetilde{q_{i j}}=q_{i i}, m_{p j}=m_{j p}=0, m_{i j}=3$ (a diagram of type super $G(3)$, with $\left.q \in \mathbb{G}_{4}\right)$.

Both possibilities follow in a similar way to the case $m_{p i}=1$.

We analyze now the second relation. As $\widetilde{q_{i j}}=-1$,

$$
\left(\left(\operatorname{ad}_{c} E_{j}\right) E_{i}\right)^{2}=q_{j i}^{2}\left(\left(\operatorname{ad}_{c} E_{i}\right) E_{j}\right)^{2}+2 q_{j i}\left(E_{i} E_{j}^{2} E_{i}+E_{j} E_{i}^{2} E_{j}\right) .
$$

By the first part $T_{p}\left(E_{i}^{2}\right), T_{p}\left(E_{j}^{2}\right) \in \mathcal{J}\left(s_{p}^{*} \chi\right)$, and as $T_{p}$ is an algebra morphism, it is enough to prove that $T_{p}\left(\left(\left(\operatorname{ad}_{c} E_{i}\right) E_{j}\right)^{2}\right) \in \mathcal{J}\left(s_{p}^{*} \chi\right)$, to conclude that also $T_{p}\left(\left(\left(\operatorname{ad}_{c} E_{j}\right) E_{i}\right)^{2}\right) \in$ $\mathcal{J}\left(s_{p}^{*} \chi\right)$, and vice versa. Moreover we need just one of these two relations in order to have a minimal set of relations.

If $p=j$, we have

$$
\begin{aligned}
T_{p}\left(\left(\left(\operatorname{ad}_{c} E_{i}\right) E_{p}\right)^{2}\right) & =\left(\underline{E}_{i, 1}^{+} \underline{F}_{p} \underline{L}_{p}^{-1}-q_{i p} \underline{F}_{p} \underline{L}_{p}^{-1} \underline{E}_{i, 1}^{+}\right)^{2} \\
& =\left(\left(\underline{F}_{p} \underline{E}_{i, 1}^{-} 2 \underline{L}_{p} \underline{E}_{i}\right) \underline{L}_{p}^{-1}-q_{i p} \underline{q}_{p p} \underline{q}_{i p} \underline{F}_{p} \underline{E}_{i, 1}^{+} \underline{L}_{p}^{-1}\right)^{2} \\
& =\left(-2 \underline{q}_{i p}^{-1} \underline{E}_{i}\right)^{2}=4 q_{i p}^{2} \underline{E}_{i}^{2} \in \mathcal{J}\left(s_{p}^{*} \chi\right),
\end{aligned}
$$

because $\underline{q}_{p p}=\underline{q}_{p i} \underline{q}_{i p}=\underline{q}_{i i}=-1$.

Now we consider $p \neq i, j$. If $m_{p i}, m_{p j} \neq 0$, we have two possibilities:

- $q_{p p}=-1, q_{i p} q_{p i} q_{j p} q_{p j}=-1$ so it is a diagram of type super $D(2,1 ; \alpha)$ ), or

- $q_{p p}=q_{i p}^{-1} q_{p i}^{-1}=-q_{j p} q_{p j} \in \mathbb{G}_{3} \cup \mathbb{G}_{4} \cup \mathbb{G}_{6}$.

For the first case, or the second when $q_{p p} \in \mathbb{G}_{4}$,

$$
T_{p}\left(\left(\left(\operatorname{ad}_{c} E_{i}\right) E_{j}\right)^{2}\right)=\left[\left(\operatorname{ad}_{\underline{c}} \underline{E}_{p}\right) \underline{E}_{i},\left(\operatorname{ad}_{\underline{c}} \underline{E}_{p}\right) \underline{E}_{j}\right]_{\underline{c}}^{2} .
$$

Using (47) and $\underline{E}_{p}^{2}$ if $\underline{q}_{p p}=-1$, or the quantum Serre relations

$$
\left(\operatorname{ad}_{\underline{c}} \underline{E}_{p}\right)^{2} \underline{E}_{i}=\left(\operatorname{ad}_{\underline{c}} \underline{E}_{p}\right)^{2} \underline{E}_{j}=0
$$


if $q_{p p} \in \mathbb{G}_{4}, \underline{E}_{i} \underline{E}_{p} \underline{E}_{j} \underline{E}_{p}$ is a linear combination of greater words, so $\left(\underline{E}_{p} \underline{E}_{i} \underline{E}_{p} \underline{E}_{j}\right)^{2}$ is also a linear combination of greater words. Then,

$$
\left[\left(\operatorname{ad}_{\underline{c}} \underline{E}_{p}\right) \underline{E}_{i},\left(\operatorname{ad}_{\underline{c}} \underline{E}_{p}\right) \underline{E}_{j}\right]_{\underline{c}}^{2} \in \mathcal{J}^{+}\left(s_{p}^{*} \chi\right)
$$

by an analogous statement to Lemma 3.4 but for powers of hyperwords, and such relation is in $I^{+}\left(s_{p}^{*} \chi\right)$.

For the remaining cases, $q_{p p} \in \mathbb{G}_{3} \cup \mathbb{G}_{6}$ and

$$
T_{p}\left(\left(\left(\operatorname{ad}_{c} E_{i}\right) E_{j}\right)^{2}\right)=\left[\left(\operatorname{ad}_{\underline{c}} \underline{E}_{p}\right) \underline{E}_{i},\left(\operatorname{ad}_{\underline{c}} \underline{E}_{p}\right)^{2} \underline{E}_{j}\right]_{\underline{c}}^{2} .
$$

We write $\left(\underline{E}_{p} \underline{E}_{i} \underline{E}_{p}^{2} \underline{E}_{j}\right)^{2}$ as a linear combination of greater words using the quantum Serre relations or (47), so

$$
T_{p}\left(\left(\left(\operatorname{ad}_{c} E_{i}\right) E_{j}\right)^{2}\right) \in \mathcal{J}^{+}\left(s_{p}^{*} \chi\right)
$$

by an analogous argument.

If $m_{p i}=1, m_{p j}=0$, we have two possibilities. If $q_{p p}=-1$, then $\widetilde{q_{p i}} \neq-1$ and $\underline{q}_{i i}=\widetilde{q_{p i}}=\widetilde{q}_{p i}^{-1}$, so (54) is a generator of $\mathcal{J}\left(s_{p}^{*} \chi\right)$, as well as $\underline{E}_{p}^{2}$ and $\underline{E}_{p j}$. By (9), we have that

$$
q_{p i} q_{p j}\left(1+\widetilde{q_{p i}}\right) \underline{E}_{p i j}^{2}=\left[\underline{E}_{p p i j}, \underline{E}_{i j}\right]_{\underline{c}}-\left[\underline{E}_{p},\left[\underline{E}_{p i j}, \underline{E}_{i j}\right]_{\underline{c}}\right]_{\underline{c}} \in \mathcal{J}\left(s_{p}^{*} \chi\right),
$$

so $T_{p}\left(E_{i j}^{2}\right)=\underline{E}_{p i j}^{2} \in \mathcal{J}\left(s_{p}^{*} \chi\right)$. If not, then $q_{p p}^{-1}=\widetilde{q_{p i}} \neq-1$, and we write $\underline{E}_{p} \underline{E}_{i} \underline{E}_{j} \underline{E}_{p} \underline{E}_{i} \underline{E}_{j}$ as a linear combination of greater words modulo $\mathcal{J}\left(s_{p}^{*} \chi\right)$, using the quantum Serre relations (observe that $\left(\underline{q}_{i j}\right)$ is twist equivalent to the original braiding). Therefore $T_{p}\left(E_{i j}^{2}\right)=\underline{E}_{p i j}^{2} \in$ $\mathcal{J}\left(s_{p}^{*} \chi\right)$.

If $m_{p i}>1, m_{p j}=0$, then $q_{p p}=-\widetilde{q_{p i}} \in \mathbb{G}_{3}$, and the proof follows in a similar way to the case $q_{p p}=-1$, but using the relation (61).

If $m_{p i}=m_{p j}=0$, the proof follows easily, because $\underline{q}_{i i}=\underline{q}_{i j} \underline{q}_{j i}=\underline{q}_{j j}=-1$, and $T_{p}\left(E_{i j}^{2}\right)=\underline{E}_{i j}^{2} \in \mathcal{J}\left(s_{p}^{*} \chi\right)$ by definition of the ideal.

Lemma 3.11. Let $i, j \in\{1, \ldots, \theta\}$ be such that $q_{i i}^{m_{i j}} \widetilde{q_{i j}}=1$. Then

$$
T_{p}\left(\left(\operatorname{ad}_{c} E_{i}\right)^{m_{i j}+1} E_{j}\right) \in \mathcal{J}\left(s_{p}^{*} \chi\right) .
$$

Proof. (i) The case $p=i$ was considered in the first part of Theorem [2.9.

(ii) Let $p=j$ : we analyze all the possible values of $m_{i p}$. If $m_{i p}=0$, then $q_{i p} q_{p i}=1$, and

$$
T_{p}\left(\left(\operatorname{ad}_{c} E_{i}\right) E_{p}\right)=\underline{E}_{i} \underline{F}_{p} \underline{L}_{p}^{-1}-q_{i p} \underline{F}_{p} \underline{L}_{p}^{-1} \underline{E}_{i}=\left(\underline{E}_{i} \underline{F}_{p}-\underline{F}_{p} \underline{E}_{i}\right) \underline{L}_{p}^{-1} \in \mathcal{J}\left(s_{p}^{*} \chi\right) .
$$

Consider $m_{i p}=1$; by (36) we have

$$
\begin{aligned}
T_{p}\left(\left(\operatorname{ad}_{c} E_{i}\right) E_{p}\right)= & \underline{E}_{i, m_{p i}}^{+} \underline{F}_{p} \underline{L}_{p}^{-1}-q_{i p} \underline{F}_{p} \underline{L}_{p}^{-1} \underline{E}_{i, m_{p i}}^{+} \\
= & \left(\underline{F}_{p} \underline{E}_{i, m_{p i}}^{+}+\left(m_{p i}\right)_{q}\left(\underline{q}_{p p}^{m_{p i}-1} \underline{q}_{p i} \underline{q}_{i p}-1\right) \underline{L}_{p} \underline{E}_{i, m_{p i}-1}^{+}\right) \underline{L}_{p}^{-1} \\
& -q_{i p} s_{p}^{+} \chi\left(m_{p i} \alpha_{p}+\alpha_{i}, \alpha_{p}\right) \underline{F}_{p} \underline{E}_{i, m_{p i}}^{+} \underline{L}_{p}^{-1} \\
= & \left(m_{p i}\right)_{\underline{q}_{p p}}\left(\underline{q}_{p p}^{m_{p i}-1} \underline{q}_{p i} \underline{q}_{i p}-1\right) s_{p}^{*} \chi\left(\left(m_{p i}-1\right) \alpha_{p}+\alpha_{i}, \alpha_{p}\right)^{-1} \underline{E}_{i, m_{p i}-1}^{+} \\
& +\underline{F}_{p} \underline{E}_{i, m_{p i}}^{+} \underline{L}_{p}^{-1}-q_{i p} \chi\left(\alpha_{i},-\alpha_{p}\right) \underline{F}_{p} \underline{E}_{i, m_{p i}}^{+} \underline{L}_{p}^{-1} \\
= & \left(m_{p i}\right)_{q_{p p}}\left(q_{p p}^{-1-m_{p i}} q_{p i}^{-1} q_{i p}^{-1}-1\right) q_{i p} q_{p p} \underline{E}_{i, m_{p i}-1}^{+} .
\end{aligned}
$$

If $m_{p i}=1$, we have by this identity and Remark 2.10 :

$$
T_{p}\left(\left(\operatorname{ad}_{c} E_{i}\right)^{2} E_{p}\right)=T_{p}\left(\left[E_{i},\left(\operatorname{ad}_{c} E_{i}\right) E_{p}\right]_{c}\right)=\left[\left(\operatorname{ad}_{\underline{c}} \underline{E}_{p}\right) \underline{E}_{i}, a_{1} \underline{E}_{i}\right]_{\underline{c}},
$$


where $a_{m_{p i}}:=\left(m_{p i}\right)_{q_{p p}}\left(q_{p p}^{-1-m_{p i}} q_{p i}^{-1} q_{i p}^{-1}-1\right) q_{i p} q_{p p} \in \mathbf{k}^{\times}$. This element is in $\mathcal{J}\left(s_{p}^{*} \chi\right)$ because $\underline{m}_{i p}=1$, so $\left(\operatorname{ad}_{\underline{c}} \underline{E}_{i}\right)^{2} \underline{E}_{p} \in \mathcal{J}\left(s_{p}^{*} \chi\right)$. We consider now $m_{p i} \geq 2$; by Lemma 3.8 ,

$$
T_{p}\left(\left(\operatorname{ad}_{c} E_{i}\right)^{2} E_{p}\right)=\left[\underline{E}_{i, m_{p i}}^{+}, a_{m_{p i}} \underline{E}_{i, m_{p i}-1}^{+}\right]_{\underline{c}} \in \mathcal{J}\left(s_{p}^{*} \chi\right) .
$$

CONSIDER NOW $m_{i p}=2$. We look at all the possible diagrams with two vertices and note that $m_{p i}=1$, or there exists $\zeta \in \mathbb{G}_{9}$ such that $q_{i i}=-\zeta, q_{i p} q_{p i}=\zeta^{7}, q_{p p}=\zeta^{3}$. In the first case, $q_{p p} \in\left\{-1, q_{i i}^{2}\right\}$, so this diagram is standard of type $B_{2}$, and (45) belongs to $\mathcal{J}\left(s_{p}^{*} \chi\right)$ by Lemma 3.8. Therefore

$$
T_{p}\left(\left(\operatorname{ad}_{c} E_{i}\right)^{3} E_{p}\right)=a_{1}\left[\left(\operatorname{ad}_{\underline{c}} \underline{E}_{p}\right) \underline{E}_{i},\left[\left(\operatorname{ad}_{\underline{c}} \underline{E}_{p}\right) \underline{E}_{i}, \underline{E}_{i}\right]_{\underline{c}}\right]_{\underline{c}} \in \mathcal{J}\left(s_{p}^{*} \chi\right) .
$$

For the second case, the braiding matrix of $s_{p}^{*} \chi$ is $\underline{q}_{i i}=-1, \underline{q}_{i p} \underline{q}_{p i}=\zeta^{8}, \underline{q}_{p p}=\zeta^{3}$. Then

$$
T_{p}\left(\left(\operatorname{ad}_{c} E_{i}\right)^{3} E_{p}\right)=\left[\left(\operatorname{ad}_{\underline{c}} \underline{E}_{p}\right)^{2} \underline{E}_{i},\left[\left(\operatorname{ad}_{\underline{c}} \underline{E}_{p}\right)^{2} \underline{E}_{i},\left(\operatorname{ad}_{\underline{c}} \underline{E}_{p}\right) \underline{E}_{i}\right]_{\underline{c}}\right]_{\underline{c}} \in \mathcal{J}\left(s_{p}^{*} \chi\right),
$$

because (65) is a generator of this ideal.

It REMAINS TO CONSIDER $m_{i p} \in\{3,4,5\}$. The unique diagram with $m_{p i}>1$ is

$$
0^{-\zeta}-\zeta^{12} \circ^{\zeta^{5}}
$$

where $\zeta \in \mathbb{G}_{15}, q_{i i}=-\zeta$ and $m_{i p}=3, m_{p i}=2$; applying $s_{p}$ we obtain

$$
\circ^{-1}-\zeta^{13} \circ^{\zeta^{5}} \text {. }
$$

By (66) we write $\underline{E}_{i} \underline{E}_{p}^{2} \underline{E}_{i} \underline{E}_{p}^{2} \underline{E}_{i} \underline{E}_{p} \underline{E}_{i}$ as a linear combination of words beginning with $\underline{E}_{p}$, or containing $\underline{E}_{p}^{3}$ as a factor, or greater than this word for the order $p<i$. Multiplying on the left by $\underline{E}_{p}^{2}$ and using that $\underline{E}_{p}^{3} \in \mathcal{J}\left(s_{p}^{*} \chi\right),\left(\underline{E}_{p}^{2} \underline{E}_{i}\right)^{3} \underline{E}_{p} \underline{E}_{i}$ can be written as a linear combination of greater words, modulo $\mathcal{J}\left(s_{p}^{*} \chi\right)$. By Lemma 3.4 we conclude that

$$
T_{p}\left(\left(\operatorname{ad}_{c} E_{i}\right)^{4} E_{p}\right)=\left[\underline{E}_{i, 2}^{+},\left[\underline{E}_{i, 2}^{+},\left[\underline{E}_{i, 2}^{+}, \underline{E}_{i, 1}^{+}\right]_{\underline{c}}\right]_{\underline{c}}\right]_{\underline{c}}=\left[\left(\underline{E}_{p}^{2} \underline{E}_{i}\right)^{3} \underline{E}_{p} \underline{E}_{i}\right]_{\underline{c}} \in \mathcal{J}\left(s_{p}^{*} \chi\right) .
$$

Finally we consider $m_{p i}=1$, so we have

$$
\begin{aligned}
s_{p}\left(m_{i p} \alpha_{i}+\alpha_{p}\right) & =m_{i p} \alpha_{i}+\left(m_{p i}-1\right) \alpha_{p} \in \Delta_{+}^{s_{p}^{*} \chi}, \\
s_{p}\left(\left(m_{i p}+1\right) \alpha_{i}+\alpha_{p}\right) & =\left(m_{i p}+1\right) \alpha_{i}+m_{p i} \alpha_{p} \notin \Delta_{+}^{s_{p}^{*} \chi} .
\end{aligned}
$$

If $q_{p p}=\underline{q}_{p p} \neq-1$ then $m_{p i}=3$ and $\left(\underline{E}_{p} \underline{E}_{i}\right)^{2} \underline{E}_{p} \underline{E}_{i}^{2}$ is a linear combination of greater words modulo $\mathcal{J}\left(s_{p}^{*} \chi\right)$, where we use first the quantum Serre relation $\left(\operatorname{ad}_{c} \underline{E}_{p}\right)^{2} \underline{E}_{i}=0$ to write $\underline{E}_{p} \underline{E}_{i} \underline{E}_{p}$ as a combination of the words $\underline{E}_{p}^{2} \underline{E}_{i}, \underline{E}_{i} \underline{E}_{p}^{2}$ and then (63), which also holds in $U\left(s_{p}^{*} \chi\right)$. By this relation,

$$
T_{p}\left(\left(\operatorname{ad}_{c} E_{i}\right)^{4} E_{p}\right)=\left[\underline{E}_{i, 1}^{+},\left[\underline{E}_{i, 1}^{+},\left[\underline{E}_{i, 1}^{+}, \underline{E}_{i}\right]_{\underline{c}}\right]_{\underline{c}}\right]_{\underline{c}} \in \mathcal{J}\left(s_{p}^{*} \chi\right) .
$$

In other case, $q_{p p}=-1$ and $m_{i p} \in\{3,4,5\}$, so we also have that

$$
T_{p}\left(\left(\operatorname{ad}_{c} E_{i}\right)^{m_{i p}+1} E_{p}\right)=\left[\underline{E}_{m_{p i} \alpha_{i}+\left(m_{p i}-1\right) \alpha_{p}},\left(\operatorname{ad}_{\underline{c}} \underline{E}_{p}\right) \underline{E}_{i}\right]_{c} \in \mathcal{J}\left(s_{p}^{*} \chi\right),
$$

by (64), (66) or (68), depending on the value of $m_{i p}$. 
(iii) Let $p \neq j$ : if $m_{p i}=0$ (i.e. $\widetilde{q_{i p}}=1$ ), then $\underline{q}_{i i}=q_{i i}, \underline{q}_{i j} \underline{q}_{j i}=\widetilde{q_{i j}}$, so $\underline{m}_{i j}=m_{i j}$, and $\left(\operatorname{ad}_{\underline{c}} \underline{E}_{i}\right)^{m_{i j}+1} \underline{E}_{j}=0$ holds in $U\left(s_{p}^{*} \chi\right)$. Moreover, in $U\left(s_{p}^{*} \chi\right)$ we have $\underline{E}_{p} \underline{E}_{i}=\underline{q}_{p i} \underline{E}_{p} \underline{E}_{i}$, so

$$
\left(\operatorname{ad}_{\underline{c}} \underline{E}_{i}\right)\left(\operatorname{ad}_{\underline{c}} \underline{E}_{p}\right) X=\underline{q}_{i p}\left(\operatorname{ad}_{\underline{c}} \underline{E}_{p}\right)\left(\operatorname{ad}_{\underline{c}} \underline{E}_{i}\right) X
$$

for any $X \in U\left(s_{p}^{*} \chi\right)$, by the second part of Lemma 3.7. By Remark 2.10 and the previous results, in $U\left(s_{p}^{*} \chi\right)$ we have

$$
\begin{aligned}
T_{p}\left(\left(\operatorname{ad}_{c} E_{i}\right)^{m_{i j}+1} E_{j}\right) & =\left(\operatorname{ad}_{\underline{c}} \underline{E}_{i}\right)^{m_{i j}+1}\left(\operatorname{ad}_{\underline{c}} \underline{E}_{p}\right)^{m_{p j}} \underline{E}_{j} \\
& =\underline{q}_{i p}^{m_{p j}\left(m_{i j}+1\right)}\left(\operatorname{ad}_{\underline{c}} \underline{E}_{p}\right)^{m_{p j}}\left(\operatorname{ad}_{\underline{c}} \underline{E}_{i}\right)^{m_{i j}+1} \underline{E}_{j}=0 .
\end{aligned}
$$

Consider now $m_{p i} \neq 0$. If $m_{i j}=m_{p j}=0$, we apply Lemma 3.7 to obtain

$$
T_{p}\left(\left(\operatorname{ad}_{c} E_{i}\right) E_{j}\right)=\left[\left(\operatorname{ad}_{\underline{c}} \underline{E}_{p}\right)^{m_{p i}} \underline{E}_{i}, \underline{E}_{j}\right]_{\underline{c}}=\left(\operatorname{ad}_{\underline{c}} \underline{E}_{p}\right)^{m_{p i}}\left(\left[\underline{E}_{i}, \underline{E}_{j}\right]_{\underline{c}}\right)=0 .
$$

where we use that $\underline{q}_{i j} \underline{q}_{j i}=\widetilde{q_{i j}}=1$, so in $U\left(s_{p}^{*} \chi\right)$ it holds that $\left[\underline{E}_{i}, \underline{E}_{j}\right]_{\underline{c}}=0$. It remains to consider the case in which $i, j$ and $p$ determine a connected diagram, and $m_{p i} \neq 0$.

First We ANALYZE THE CASE $m_{i j}=0, m_{p j} \neq 0$. If $q_{p p}=-1$ it follows that $m_{p i}=$ $m_{p j}=1$. Then $\underline{q}_{i j} \underline{q}_{j i}=q_{i p} q_{p i} q_{j p} q_{p j}$, and $\underline{E}_{p} \underline{E}_{i} \underline{E}_{p} \underline{E}_{j}$ is a linear combination of greater words for the order $p<i<j$, modulo $\mathcal{J}\left(s_{p}^{*} \chi\right)$ :

- if $\underline{q}_{i j} \underline{q}_{j i}=1$, it follows from (44),

- if $\underline{q}_{i j} \underline{q}_{j i} \neq 1$, we write $\underline{E}_{i} \underline{E}_{p} \underline{E}_{j}$ as a linear combination of other words by (47), where those words are greater than $\underline{E}_{i} \underline{E}_{p} \underline{E}_{j}$ or begin with $\underline{E}_{p}$, so we multiply on the left by $\underline{E}_{p}$ and use that $\underline{E}_{p}^{2} \in \mathcal{J}\left(s_{p}^{*} \chi\right)$.

In this way, $T_{p}\left(\left(\operatorname{ad}_{c} E_{i}\right) E_{j}\right)=\left[\left(\operatorname{ad}_{\underline{c}} \underline{E}_{p}\right) \underline{E}_{i},\left(\operatorname{ad}_{\underline{c}} \underline{E}_{p}\right) \underline{E}_{j}\right]_{\underline{c}} \in \mathcal{J}\left(s_{p}^{*} \chi\right)$. If $m_{p i}=m_{p j}=1$ and $q_{p p} \neq-1$, then $\left(\operatorname{ad}_{\underline{c}} \underline{E}_{p}\right)^{2} \underline{E}_{i},\left(\operatorname{ad}_{\underline{c}} \underline{E}_{p}\right)^{2} \underline{E}_{j} \in \mathcal{J}\left(s_{p}^{*} \chi\right)$; by these relations and $\left(\operatorname{ad}_{\underline{c}} \underline{E}_{i}\right) \underline{E}_{j}$, $\underline{E}_{p} \underline{E}_{i} \underline{E}_{p} \underline{E}_{j}$ can be written as a linear combination of greater words for the order $p<i<j$, modulo $\mathcal{J}\left(s_{p}^{*} \chi\right)$, and also $T_{p}\left(\left(\operatorname{ad}_{c} E_{i}\right) E_{j}\right) \in \mathcal{J}\left(s_{p}^{*} \chi\right)$ in this case.

If $m_{p j}=1$ and $m_{p i}>1$ (or analogously, $m_{p j}>1, m_{p i}=1$ ), then $q_{p p} q_{p j} q_{j p}=1$, and $q_{p p} \neq-1$. Note that

$$
\widetilde{q_{i j}}=s_{p}^{*} \chi\left(\alpha_{i}, \alpha_{j}\right) s_{p}^{*} \chi\left(\alpha_{j}, \alpha_{i}\right)=q_{p p}^{m_{p i}} q_{p i} q_{i p} .
$$

If $q_{p p}^{m_{p i}} q_{p i} q_{i p} \neq 1$, then (477) holds in $U\left(s_{p}^{*} \chi\right)$, so we can write $\underline{E}_{i} \underline{E}_{p} \underline{E}_{j}$ as a linear combination of other words, greater than $\underline{E}_{i} \underline{E}_{p} \underline{E}_{j}$ for the order $p<i<j$, or beginning with $\underline{E}_{p}$. Multiplying on the left by $\underline{E}_{p}^{m_{p i}}$, we express $\underline{E}_{p}^{m_{i j}} \underline{E}_{i} \underline{E}_{p} \underline{E}_{j}$ as a linear combination of greater words, using that $\underline{E}_{p}^{m_{p i}+1} \in \mathcal{J}\left(s_{p}^{*} \chi\right)$, or $\left(\operatorname{ad}_{\underline{c}} \underline{E}_{p}\right)^{m_{p i}+1} \underline{E}_{i} \in \mathcal{J}\left(s_{p}^{*} \chi\right)$, so

$$
T_{p}\left(\left(\operatorname{ad}_{c} E_{i}\right) E_{j}\right)=\left[\left(\operatorname{ad}_{\underline{c}} \underline{E}_{p}\right)^{m_{p i}} \underline{E}_{i},\left(\operatorname{ad}_{\underline{c}} \underline{E}_{p}\right) \underline{E}_{j}\right]_{\underline{c}} \in \mathcal{J}\left(s_{p}^{*} \chi\right) .
$$

If $q_{p p}^{m_{p i}} q_{p i} q_{i p}=1$ and $q_{p p}^{m_{p i}+1} \neq 1, \underline{E}_{p}^{m_{i j}} \underline{E}_{i} \underline{E}_{p} \underline{E}_{j}$ is written as a linear combination of greater words for the same order using $\left(\operatorname{ad}_{\underline{c}} \underline{E}_{p}\right)^{m_{p i}+1} \underline{E}_{i},\left(\operatorname{ad}_{\underline{c}} \underline{E}_{p}\right)^{2} \underline{E}_{j}$ and $\left(\operatorname{ad}_{\underline{c}} \underline{E}_{i}\right) \underline{E}_{j}$, so we obtain the same conclusion. If $q_{p p}^{m_{p i}} q_{p i} q_{i p}=1$ and $q_{p p}^{m_{p i}+1}=1$, then $m_{p i}=2$ or $m_{p i}=3$, and the conclusion follows from (50) or (53), respectively.

If $m_{p i}, m_{p j}>1$, there is only one possibility: $m_{p i}=m_{p j}=2$. The proof is as above, expressing $\underline{E}_{p}^{2} \underline{E}_{i} \underline{E}_{p}^{2} \underline{E}_{j}$ as a linear combination of greater words in the two possible cases: if $q_{p p} \notin \mathbb{G}_{3}$, using the quantum Serre relations; if $q_{p p} \in \mathbb{G}_{3}$, by (60) and $\underline{E}_{p}^{3}$.

We CONSIDER NOW $m_{p j}=0, m_{i j} \neq 0$. Note that $m_{i j} \leq 3$, because we have a connected diagram with three vertices and $q_{i i} \neq-1: q_{i i}^{m_{i j}+1} \neq 1$. If $m_{i j}=3$, it corresponds 
to a diagram of type super $G(3)$ :

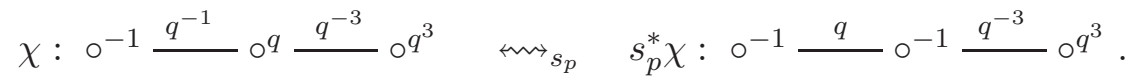

Using (49), $\underline{E}_{i}\left(\underline{E}_{p} \underline{E}_{i}\right)^{3} \underline{E}_{j}$ can be written as a linear combination of other words modulo $\mathcal{J}\left(s_{p}^{*} \chi\right)$, which are greater than this word for the order $p<i<j$, or begin with $\underline{E}_{p}$ (recall that $\left.\underline{E}_{i}^{2} \in \mathcal{J}\left(s_{p}^{*} \chi\right)\right)$. Multiplying on the left by $\underline{E}_{p},\left(\underline{E}_{p} \underline{E}_{i}\right)^{4} \underline{E}_{j}$ is expressed as a linear combination of greater words, modulo $\mathcal{J}\left(s_{p}^{*} \chi\right)$, using that $\underline{E}_{p}^{2} \in \mathcal{J}\left(s_{p}^{*} \chi\right)$. By Lemma 3.4,

$$
\begin{aligned}
T_{p}\left(\left(\operatorname{ad}_{c} E_{i}\right)^{4} E_{j}\right) & =\left[\left(\operatorname{ad}_{\underline{c}} \underline{E}_{p}\right) \underline{E}_{i},\left[\left(\operatorname{ad}_{\underline{c}} \underline{E}_{p}\right) \underline{E}_{i},\left[\left(\operatorname{ad}_{\underline{c}} \underline{E}_{p}\right) \underline{E}_{i},\left(\operatorname{ad}_{\underline{c}} \underline{E}_{p}\right)\left(\operatorname{ad}_{\underline{c}} \underline{E}_{i}\right) \underline{E}_{j}\right]_{\underline{c}}\right]_{\underline{c}}\right]_{\underline{c}} \\
& =\left[\left(\underline{E}_{p} \underline{E}_{i}\right)^{4} \underline{E}_{j}\right]_{\underline{c}} \in \mathcal{J}\left(s_{p}^{*} \chi\right) .
\end{aligned}
$$

If $m_{i j}=2$, then $m_{p i}=2$ for the diagram

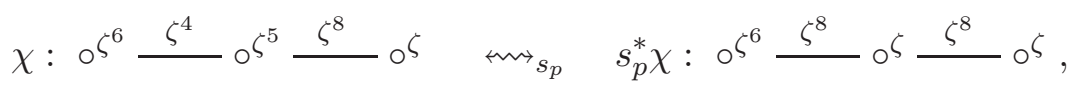

where $\zeta \in \mathbb{G}_{9}$, or $m_{p i}=1$. In the first case, we use (51), $\underline{E}_{p}^{3} \in \mathcal{J}\left(s_{p}^{*} \chi\right)$ and the quantum Serre relations, and call $c=s_{p}^{*} \chi\left(4 \alpha_{p}+2 \alpha_{i}+\alpha_{j}, \alpha_{p}+\alpha_{i}\right)=\chi\left(2 \alpha_{i}+\alpha_{j}, \alpha_{p}+\alpha_{i}\right)$, so

$$
\begin{gathered}
T_{p}\left(\left(\operatorname{ad}_{c} E_{i}\right)^{3} E_{j}\right)=\left[\left(\operatorname{ad}_{\underline{c}} \underline{E}_{p}\right)^{2} \underline{E}_{i},\left[\left(\operatorname{ad}_{\underline{c}} \underline{E}_{p}\right)^{2} \underline{E}_{i},\left(\operatorname{ad}_{\underline{c}} \underline{E}_{p}\right)^{2}\left(\operatorname{ad}_{\underline{c}} \underline{E}_{i}\right) \underline{E}_{j}\right]_{\underline{c}}\right]_{\underline{c}} \\
=\left(\operatorname{ad}_{\underline{c}} \underline{E}_{p}\right)\left(\left[\left(\operatorname{ad}_{\underline{c}} \underline{E}_{p}\right) \underline{E}_{i},\left[\left(\operatorname{ad}_{\underline{c}} \underline{E}_{p}\right)^{2} \underline{E}_{i},\left(\operatorname{ad}_{\underline{c}} \underline{E}_{p}\right)^{2}\left(\operatorname{ad}_{\underline{c}} \underline{E}_{i}\right) \underline{E}_{j}\right]_{\underline{c}}\right]_{\underline{c}}\right) \\
=-c\left(\operatorname{ad}_{\underline{c}} \underline{E}_{p}\right)\left(\left[\left[\underline{E}_{p p i}, \underline{E}_{p p i j}\right], \underline{E}_{p i}\right]_{\underline{c}}\right) \in \mathcal{J}\left(s_{p}^{*} \chi\right) .
\end{gathered}
$$

If $m_{p i}=1$ and $q_{p p} \neq-1$, using $\left(\operatorname{ad}_{\underline{c}} \underline{E}_{p}\right)^{2} \underline{E}_{i}$ we write $\underline{E}_{p} \underline{E}_{i} \underline{E}_{p} \underline{E}_{i} \underline{E}_{j} \underline{E}_{i}$ as a linear combination of greater words and $\underline{E}_{p}^{2} \underline{E}_{i}^{2} \underline{E}_{j} \underline{E}_{i}$, for the order induced by $p<i<j$, modulo $\mathcal{J}\left(s_{p}^{*} \chi\right)$. Using now $\left(\operatorname{ad}_{\underline{c}} \underline{E}_{p}\right) \underline{E}_{j}$, and $\left(\operatorname{ad}_{\underline{c}} \underline{E}_{i}\right)^{3} \underline{E}_{j}$ when ${\widetilde{q_{i p}}}^{3} \neq 1$, or (50) in other case, $\underline{E}_{p} \underline{E}_{i} \underline{E}_{p} \underline{E}_{i} \underline{E}_{j} \underline{E}_{i}$ is expressed as a linear combination of greater words modulo $\mathcal{J}\left(s_{p}^{*} \chi\right)$. If $m_{p i}=1$ and $q_{p p}=-1$, then (48) is a generator of $\mathcal{J}\left(s_{p}^{*} \chi\right)$, so

$$
\left[\left[\left(\operatorname{ad}_{\underline{c}} \underline{E}_{p}\right) \underline{E}_{i},\left(\operatorname{ad}_{\underline{c}} \underline{E}_{p}\right)\left(\operatorname{ad}_{\underline{c}} \underline{E}_{i}\right) \underline{E}_{j}\right]_{\underline{c}}, \underline{E}_{i}\right]_{\underline{c}} \in \mathcal{J}\left(s_{p}^{*} \chi\right),
$$

or (45) (considered for the pair $p, i$ ) is a generator of the ideal. In any case we have that

$$
\begin{aligned}
T_{p}\left(\left(\operatorname{ad}_{c} E_{i}\right)^{3} E_{j}\right) & =\left[\left(\operatorname{ad}_{\underline{c}} \underline{E}_{p}\right) \underline{E}_{i},\left[\left(\operatorname{ad}_{\underline{c}} \underline{E}_{p}\right) \underline{E}_{i},\left(\operatorname{ad}_{\underline{c}} \underline{E}_{p}\right)\left(\operatorname{ad}_{\underline{c}} \underline{E}_{i}\right) \underline{E}_{j}\right]_{\underline{c}}\right]_{\underline{c}} \\
& =\left(\operatorname{ad}_{\underline{c}} \underline{E}_{p}\right)\left[\underline{E}_{i},\left[\left(\operatorname{ad}_{\underline{c}} \underline{E}_{p}\right) \underline{E}_{i},\left(\operatorname{ad}_{\underline{c}} \underline{E}_{p}\right)\left(\operatorname{ad}_{\underline{c}} \underline{E}_{i}\right) \underline{E}_{j}\right]_{\underline{c}}\right]_{\underline{c}} \in \mathcal{J}\left(s_{p}^{*} \chi\right) .
\end{aligned}
$$

Now we fix $m_{i j}=1$. If $m_{p i}=1$, we analyze each different possible diagram.

- If $q_{p p} \neq-1$, then $s_{p}^{*} \chi$ is twist equivalent to $\chi$ (restricted to the vertices $p, i, j$ ), and $\underline{E}_{p} \underline{E}_{i} \underline{E}_{p} \underline{E}_{i} \underline{E}_{j}$ can be expressed as a linear combination of greater words modulo $\mathcal{J}\left(s_{p}^{*} \chi\right)$, using the quantum Serre relations

$$
\left(\operatorname{ad}_{\underline{c}} \underline{E}_{p}\right)^{2} \underline{E}_{i}=\left(\operatorname{ad}_{\underline{c}} \underline{E}_{i}\right)^{2} \underline{E}_{j}=\left(\operatorname{ad}_{\underline{c}} \underline{E}_{p}\right) \underline{E}_{j}=0 \text {. }
$$

- If $q_{p p}=-1$ and $q_{i i} q_{i p} q_{p i}=1$, then $\underline{q}_{i i}=-1$ and $\underline{q}_{i p} \underline{q}_{p i} \underline{q}_{i j} \underline{q}_{j i}=1$. In this way (44) is a generator of the ideal, and by Lemma 3.7 .

$$
\begin{aligned}
T_{p}\left(\left(\operatorname{ad}_{c} E_{i}\right)^{2} E_{j}\right) & =\left[\left(\operatorname{ad}_{\underline{c}} \underline{E}_{p}\right) \underline{E}_{i},\left(\operatorname{ad}_{\underline{c}} \underline{E}_{p}\right)\left(\operatorname{ad}_{\underline{c}} \underline{E}_{i}\right) \underline{E}_{j}\right]_{\underline{c}} \\
& =\left(\operatorname{ad}_{\underline{c}} \underline{E}_{p}\right)\left(\left[\underline{E}_{i},\left(\operatorname{ad}_{\underline{c}} \underline{E}_{p}\right)\left(\operatorname{ad}_{\underline{c}} \underline{E}_{i}\right) \underline{E}_{j}\right]_{\underline{c}}\right) \in \mathcal{J}\left(s_{p}^{*} \chi\right) .
\end{aligned}
$$


- If $q_{p p}=-1$ and $q_{i i} q_{i p} q_{p i} \neq 1$, then $\underline{q}_{p j} \underline{q}_{j p}=q_{p j} q_{j p}=1, \underline{q}_{i j} \underline{q}_{j i}=\widetilde{q_{i j}} \neq-1$ and

$$
\underline{q}_{i i}^{-1}=q_{p p}^{-1} q_{i p}^{-1} q_{p i}^{-1} q_{i i}^{-1}=-\underline{q}_{i p} \underline{q}_{p i} \underline{q}_{i j} \underline{q}_{j i} \neq-1,
$$

so (54) or (55) are generators of $\mathcal{J}\left(s_{p}^{*} \chi\right)$, and then $T_{p}\left(\left(\operatorname{ad}_{c} E_{i}\right)^{2} E_{j}\right) \in \mathcal{J}\left(s_{p}^{*} \chi\right)$.

Now we fix $m_{p i} \neq 1$. The possible connected diagrams of rank three with these conditions must verify $m_{p i}=2, m_{i p}=1$. Using the quantum Serre relations $\left(\operatorname{ad}_{\underline{c}} \underline{E}_{p}\right) \underline{E}_{j}=$ $\left(\operatorname{ad}_{\underline{c}} \underline{E}_{p}\right)^{3} \underline{E}_{i}=0$ if $q_{p p}^{3} \neq-1$, or (46), (52) depending on the case, $\underline{E}_{p}^{2} \underline{E}_{i} \underline{E}_{j} \underline{E}_{p} \underline{E}_{i}$ can be expressed as a linear combination of greater words for the order $p<i<j$, and by Lemma 3.4 ,

$$
\left[\left(\operatorname{ad}_{\underline{c}} \underline{E}_{p}\right)^{2}\left(\operatorname{ad}_{\underline{c}} \underline{E}_{i}\right) \underline{E}_{j},\left(\operatorname{ad}_{\underline{c}} \underline{E}_{p}\right) \underline{E}_{i}\right]_{\underline{c}} \in \mathcal{J}\left(s_{p}^{*} \chi\right) .
$$

By Lemma (3.7) we conclude that

$$
\begin{aligned}
T_{p}\left(\left(\operatorname{ad}_{c} E_{i}\right)^{2} E_{j}\right) & =\left[\left(\operatorname{ad}_{\underline{c}} \underline{E}_{p}\right)^{2} \underline{E}_{i},\left(\operatorname{ad}_{\underline{c}} \underline{E}_{p}\right)^{2}\left(\operatorname{ad}_{\underline{c}} \underline{E}_{i}\right) \underline{E}_{j}\right]_{\underline{c}} \\
& =\left(\operatorname{ad}_{\underline{c}} \underline{E}_{p}\right)\left(\left[\left(\operatorname{ad}_{\underline{c}} \underline{E}_{p}\right) \underline{E}_{i},\left(\operatorname{ad}_{\underline{c}} \underline{E}_{p}\right)^{2}\left(\operatorname{ad}_{\underline{c}} \underline{E}_{i}\right) \underline{E}_{j}\right]_{\underline{c}}\right) \in \mathcal{J}\left(s_{p}^{*} \chi\right) .
\end{aligned}
$$

FinALLY WE CONSIDER $m_{i j}, m_{p j} \neq 0$, so each pair of vertices is connected. If $m_{i j}=2$, there is just one possibility,

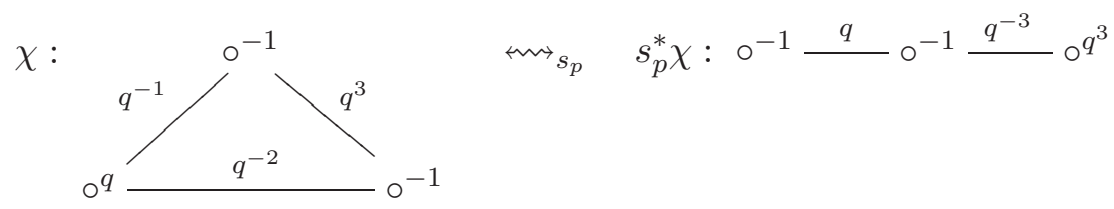

which is a diagram of type super $G(3)$. By (49) and Lemma 3.5 we have that

$$
T_{p}\left(\left(\operatorname{ad}_{\underline{c}} E_{i}\right)^{3} E_{j}\right)=\left[\left(\operatorname{ad}_{\underline{c}} \underline{E}_{p}\right) \underline{E}_{i},\left[\left(\operatorname{ad}_{\underline{c}} \underline{E}_{p}\right) \underline{E}_{i},\left[\left(\operatorname{ad}_{\underline{c}} \underline{E}_{p}\right) \underline{E}_{i},\left(\operatorname{ad}_{\underline{c}} \underline{E}_{p}\right) \underline{E}_{j}\right]_{\underline{c}}\right]_{\underline{c}}\right]_{\underline{c}} \in \mathcal{J}\left(s_{p}^{*} \chi\right) .
$$

The remaining case is $m_{i j}=1$. If $m_{p i}=m_{p j}=1$, there are two possible cases:

- $q_{p p}=-1$; in this case (48) is a generator of the ideal $\mathcal{J}\left(s_{p}^{*} \chi\right)$ by definition, and by Lemma 3.5 we have that

$$
T_{p}\left(\left(\operatorname{ad}_{c} E_{i}\right)^{2} E_{j}\right)=\left[\left(\operatorname{ad}_{\underline{c}} \underline{E}_{p}\right) \underline{E}_{i},\left[\left(\operatorname{ad}_{\underline{c}} \underline{E}_{p}\right) \underline{E}_{i},\left(\operatorname{ad}_{\underline{c}} \underline{E}_{p}\right) \underline{E}_{j}\right]_{\underline{c}}\right]_{\underline{c}} \in \mathcal{J}\left(s_{p}^{*} \chi\right) .
$$

- $q_{p p} \neq-1, q_{p p} q_{p i} q_{i p}=q_{p p} q_{p j} q_{j p}=1 ; s_{p}^{*} \chi$ is twist equivalent to $\chi$, so (47) is a generator of $\mathcal{J}\left(s_{p}^{*} \chi\right)$. Using also the quantum Serre relations $\left(\operatorname{ad}_{\underline{c}} \underline{E}_{p}\right)^{2} \underline{E}_{i},\left(\operatorname{ad}_{\underline{c}} \underline{E}_{p}\right)^{2} \underline{E}_{j}$, $\left(\operatorname{ad}_{\underline{c}} \underline{E}_{i}\right)^{2} \underline{E}_{j}, \underline{E}_{p} \underline{E}_{i} \underline{E}_{p} \underline{E}_{i} \underline{E}_{p} \underline{E}_{j}$ is written as a linear combination of greater words, modulo $\mathcal{J}\left(s_{p}^{*} \chi\right)$, so as before $T_{p}\left(\left(\operatorname{ad}_{c} E_{i}\right)^{2} E_{j}\right) \in \mathcal{J}\left(s_{p}^{*} \chi\right)$.

It remains to consider the following braiding:

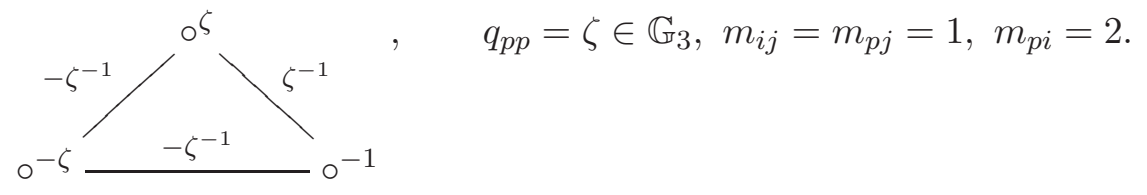

The diagram of $s_{p}^{*} \chi$ is $\circ^{-1}-1$ is expressed as a linear combination of other words of the same $\mathbb{Z}^{\theta}$-degree. Multiplying on the left by $\underline{E}_{p}^{2}$, on the right by $\underline{E}_{j}$, and using that $\underline{E}_{p}^{3}=0, \underline{E}_{p}^{2} \underline{E}_{i} \underline{E}_{p}^{2} \underline{E}_{i} \underline{E}_{p} \underline{E}_{j}$ can be written as a linear combination of greater words, so

$$
T_{p}\left(\left(\operatorname{ad}_{\underline{c}} E_{i}\right)^{2} E_{j}\right)=\left[\left(\operatorname{ad}_{\underline{c}} \underline{E}_{p}\right)^{2} \underline{E}_{i},\left[\left(\operatorname{ad}_{\underline{c}} \underline{E}_{p}\right)^{2} \underline{E}_{i},\left(\operatorname{ad}_{\underline{c}} \underline{E}_{p}\right) \underline{E}_{j}\right]_{\underline{c}}\right]_{\underline{c}} \in \mathcal{J}\left(s_{p}^{*} \chi\right) .
$$


Therefore we analyze all the cases and the proof is completed.

Lemma 3.12. Let $i, j, k \in\{1, \ldots, \theta\}$ be such that $q_{j j}=-1, \widetilde{q_{i k}}=\widetilde{q_{i j}} \widetilde{q_{j k}}=1$. Then,

$$
T_{p}\left(\left[E_{i j k}, E_{j}\right]_{c}\right) \in \mathcal{J}\left(s_{p}^{*} \chi\right) .
$$

Proof. Note that in $U(\chi)$ we have the following identity, using the condition on the scalars, (9) and $\left(\operatorname{ad}_{c} E_{i}\right) E_{k}=E_{j}^{2}=0$ :

$$
\left[E_{i j k}, E_{j}\right]_{c}=q_{i j} q_{k j}\left[E_{j}, E_{i j k}\right]_{c}=q_{i j} q_{k j}\left[E_{j i}, E_{j k}\right]_{c},
$$

so it is enough to prove that one of these relations is applied in $\mathcal{J}\left(s_{p}^{*} \chi\right)$ by $T_{p}$ for each possible diagram. Let $p=j$. Note that $\underline{q}_{p p}=-1, \underline{q}_{i p} \underline{q}_{p i} \underline{q}_{p k} \underline{q}_{k p}=\underline{q}_{i k} \underline{q}_{k i}=1$, so $\left(\operatorname{ad}_{\underline{c}} \underline{E}_{i}\right) \underline{E}_{k}$ and (44) are generators of $\mathcal{J}\left(s_{p}^{*} \chi\right)$. By (36) we have:

$$
\begin{aligned}
T_{p}\left(\left[E_{i p k}, E_{p}\right]_{c}\right)= & {\left[a_{1} \underline{E}_{i},\left(\operatorname{ad}_{\underline{c}} \underline{E}_{p}\right) \underline{E}_{k}\right]_{\underline{c}} \underline{F}_{p} \underline{L}_{p}^{-1}+q_{i p} q_{k p} \underline{F}_{p} \underline{L}_{p}^{-1}\left[a_{1} \underline{E}_{i},\left(\operatorname{ad}_{\underline{c}} \underline{E}_{p}\right) \underline{E}_{k}\right]_{\underline{c}} } \\
= & a_{1}\left(\underline{q}_{i p}^{-1} \underline{q}_{p p}^{-1} \underline{q}_{k p}^{-1}+q_{i p} q_{k p}\right) \underline{F}_{p} \underline{L}_{p}^{-1}\left[\underline{E}_{i},\left(\operatorname{ad}_{\underline{c}} \underline{E}_{p}\right) \underline{E}_{k}\right]_{\underline{c}} \\
& +a_{1} q_{p k}^{-1}\left(1-q_{p k} q_{k p}\right)\left(\underline{E}_{i} \underline{E}_{k}+q_{i p} q_{p k} q_{i k} \underline{E}_{k} \underline{E}_{i}\right) \\
= & a_{1} q_{p k}^{-1}\left(1-q_{p k} q_{k p}\right)\left(\operatorname{ad}_{\underline{c}} \underline{E}_{i}\right) \underline{E}_{k} \in \mathcal{J}\left(s_{p}^{*} \chi\right) .
\end{aligned}
$$

Let $p=i$, which is analogous to the case $p=k$. By (69) and (71),

$$
T_{p}\left(\left[E_{j p}, E_{j k}\right]_{c}\right)=\left[a_{m_{p j}}\left(\operatorname{ad}_{\underline{c}} \underline{E}_{p}\right)^{m_{p j}-1} \underline{E}_{j},\left(\operatorname{ad}_{\underline{c}} \underline{E}_{p}\right)^{m_{p j}}\left(\operatorname{ad}_{\underline{c}} \underline{E}_{j}\right) \underline{E}_{k}\right]_{\underline{c}} .
$$

Note that $m_{p j}=1,2$. If $m_{p j}=1, q_{p p} \neq-1$ or $m_{p j}=2, q_{p p} \notin \mathbb{G}_{3}$, then $q_{p p}^{m_{p j}} q_{p j} q_{j p}=1$. In this case, $-\underline{q}_{j j}=\underline{q}_{p k} \underline{q}_{k p}=\underline{q}_{p j} \underline{q}_{j p} \underline{q}_{k j} \underline{q}_{j k}=1$, so $\left(\operatorname{ad}_{\underline{c}} \underline{E}_{p}\right)^{m_{p j}+1} \underline{E}_{j}=0=\left(\operatorname{ad}_{\underline{c}} \underline{E}_{p}\right) \underline{E}_{k}$. Note also that (44) is a generator of $\mathcal{J}\left(s_{p}^{*} \chi\right)$, hence $T_{p}\left(\left[\left(\operatorname{ad}_{c} E_{j}\right) E_{i},\left(\operatorname{ad}_{c} E_{j}\right) E_{k}\right]_{c}\right) \in \mathcal{J}\left(s_{p}^{*} \chi\right)$. If $q_{p p}=-1$, then

$$
\underline{q}_{j j} \underline{q}_{j p} \underline{q}_{p j}=\underline{q}_{j j} \underline{q}_{j k} \underline{q}_{k j}=1, \quad \underline{q}_{p k} \underline{q}_{k p}=1,
$$

hence in $U\left(s_{p}^{*} \chi\right),\left(\operatorname{ad}_{\underline{c}} \underline{E}_{j}\right)^{2} \underline{E}_{p}=\left(\operatorname{ad}_{\underline{c}} \underline{E}_{j}\right)^{2} \underline{E}_{k}=0$ if $\underline{q}_{j j} \neq-1$, or (44) if $\underline{q}_{j j}=-1$ : in this way,

$$
T_{p}\left(\left[E_{j p}, E_{j k}\right]_{c}\right)=a_{1}\left[\underline{E}_{j},\left(\operatorname{ad}_{\underline{c}} \underline{E}_{p}\right)\left(\operatorname{ad}_{\underline{c}} \underline{E}_{j}\right) \underline{E}_{k}\right]_{\underline{c}} \in \mathcal{J}\left(s_{p}^{*} \chi\right) .
$$

The remaining case is $q_{p p} \in \mathbb{G}_{3}$ : by (46) we obtain that

$$
T_{p}\left(\left[E_{j p}, E_{j k}\right]_{c}\right)=a_{2}\left[\left(\operatorname{ad}_{\underline{c}} \underline{E}_{p}\right) \underline{E}_{j},\left(\operatorname{ad}_{\underline{c}} \underline{E}_{p}\right)^{2}\left(\operatorname{ad}_{\underline{c}} \underline{E}_{j}\right) \underline{E}_{k}\right]_{\underline{c}} \in \mathcal{J}\left(s_{p}^{*} \chi\right) .
$$

Finally take $p \neq i, j, k$. First, the proof is trivial if $p$ is not connected with $i, j, k$, because in such case $s_{p}^{*} \chi$ is twist equivalent to $\chi$, and then

$$
T_{p}\left(\left[E_{i j k}, E_{j}\right]_{c}\right)=\left[\underline{E}_{i j k}, \underline{E}_{j}\right]_{\underline{c}} \in \mathcal{J}\left(s_{p}^{*} \chi\right) .
$$

Now, if $p$ is connected just with $i$ (or analogously, just with $k$ ), we have

$$
\underline{q}_{j j}=-1, \quad \underline{q}_{j i} \underline{q}_{i j} \underline{q}_{j k} \underline{q}_{k j}=1, \quad \underline{q}_{i k} \underline{q}_{k i}=1,
$$

and by Lemma 3.7 ;

$$
T_{p}\left(\left[E_{i j k}, E_{j}\right]_{c}\right)=\left[\left(\operatorname{ad}_{\underline{c}} \underline{E}_{p}\right)^{m_{p i}}\left(\underline{E}_{i j k}\right), \underline{E}_{j}\right]_{\underline{c}}=\left(\operatorname{ad}_{\underline{c}} \underline{E}_{p}\right)^{m_{p i}}\left(\left[\underline{E}_{i j k}, \underline{E}_{j}\right]_{\underline{c}}\right) \in \mathcal{J}\left(s_{p}^{*} \chi\right) .
$$

If $p$ is connected just with $j$, then $\widetilde{q_{p j}} \in\left\{\widetilde{q_{i j}}, \widetilde{q_{j k}}\right\}$, and $m_{p j}=1$. We assume that $\widetilde{q_{p j}}=$ $\widetilde{q_{i j}}={\widetilde{q_{k j}}}^{-1}$. If $q_{p p} \widetilde{q_{i p}}=1, s_{p}^{*} \chi$ is twist equivalent to $\chi$; in other case, $q_{p p}=-1$, and then

$$
\underline{q}_{j j}=q_{p j} q_{j p}, \quad \underline{q}_{p j} \underline{q}_{j p}=q_{p j}^{-1} q_{j p}^{-1}=q_{k j} q_{j k}=\underline{q}_{k j} \underline{q}_{j k},
$$


so in both cases $\left[\left(\operatorname{ad}_{\underline{c}} \underline{E}_{p}\right)\left(\operatorname{ad}_{\underline{c}} \underline{E}_{j}\right) \underline{E}_{k}, \underline{E}_{j}\right]_{\underline{c}} \in \mathcal{J}\left(s_{p}^{*} \chi\right)$. Therefore $\underline{E}_{p} \underline{E}_{j} \underline{E}_{k} \underline{E}_{p} \underline{E}_{j} \underline{E}_{i}$ is a linear combination of greater words (for the order $p<j<k<i$ ), so we have that

$$
T_{p}\left(\left[\left(\operatorname{ad}_{c} E_{j}\right) E_{k},\left(\operatorname{ad}_{c} E_{j}\right) E_{i}\right]_{c}\right)=\left[\left(\operatorname{ad}_{\underline{c}} \underline{E}_{p}\right)\left(\operatorname{ad}_{\underline{c}} \underline{E}_{j}\right) \underline{E}_{k},\left(\operatorname{ad}_{\underline{c}} \underline{E}_{p}\right)\left(\operatorname{ad}_{\underline{c}} \underline{E}_{j}\right) \underline{E}_{i}\right]_{\underline{c}} \in \mathcal{J}\left(s_{p}^{*} \chi\right) .
$$

The remaining case is that $p$ is connected with two consecutive vertices. We can assume that $p$ is connected with $i$ and $j$. There exist six possible diagrams satisfying these conditions. For two of them, the diagram of $s_{p}^{*} \chi$ is the same.

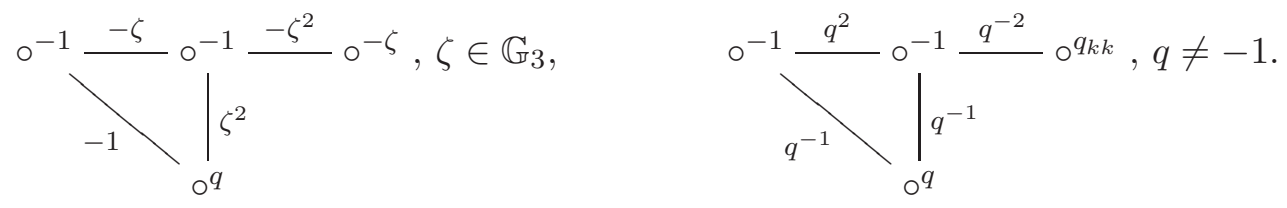

In the second case, $m_{p i}=m_{p j}=1$. Consider the order $p<j<i<k$ :

$$
T_{p}\left(\left[E_{j i}, E_{j k}\right]_{c}\right)=\left[\left[\underline{E}_{p j}, \underline{E}_{p i}\right]_{\underline{c}},\left[\underline{E}_{p j}, \underline{E}_{k}\right]_{\underline{c}}\right]_{\underline{c}}=\left[\underline{E}_{p} \underline{E}_{j} \underline{E}_{p} \underline{E}_{i} \underline{E}_{p} \underline{E}_{j} \underline{E}_{k}\right] .
$$

Note that $\underline{E}_{j} \underline{E}_{p} \underline{E}_{i}$ is a linear combination of other words of the same degree by (47), where those words are greater than $\underline{E}_{j} \underline{E}_{p} \underline{E}_{i}$ or begin with $\underline{E}_{p}$. In all the cases we conclude that $T_{p}\left(\left[E_{j i}, E_{j k}\right]_{c}\right) \in \mathcal{J}\left(s_{p}^{*} \chi\right)$. The proof for the first case is analogous.

For the remaining four diagrams, we write also the diagram corresponding to $s_{p}^{*} \chi$ :
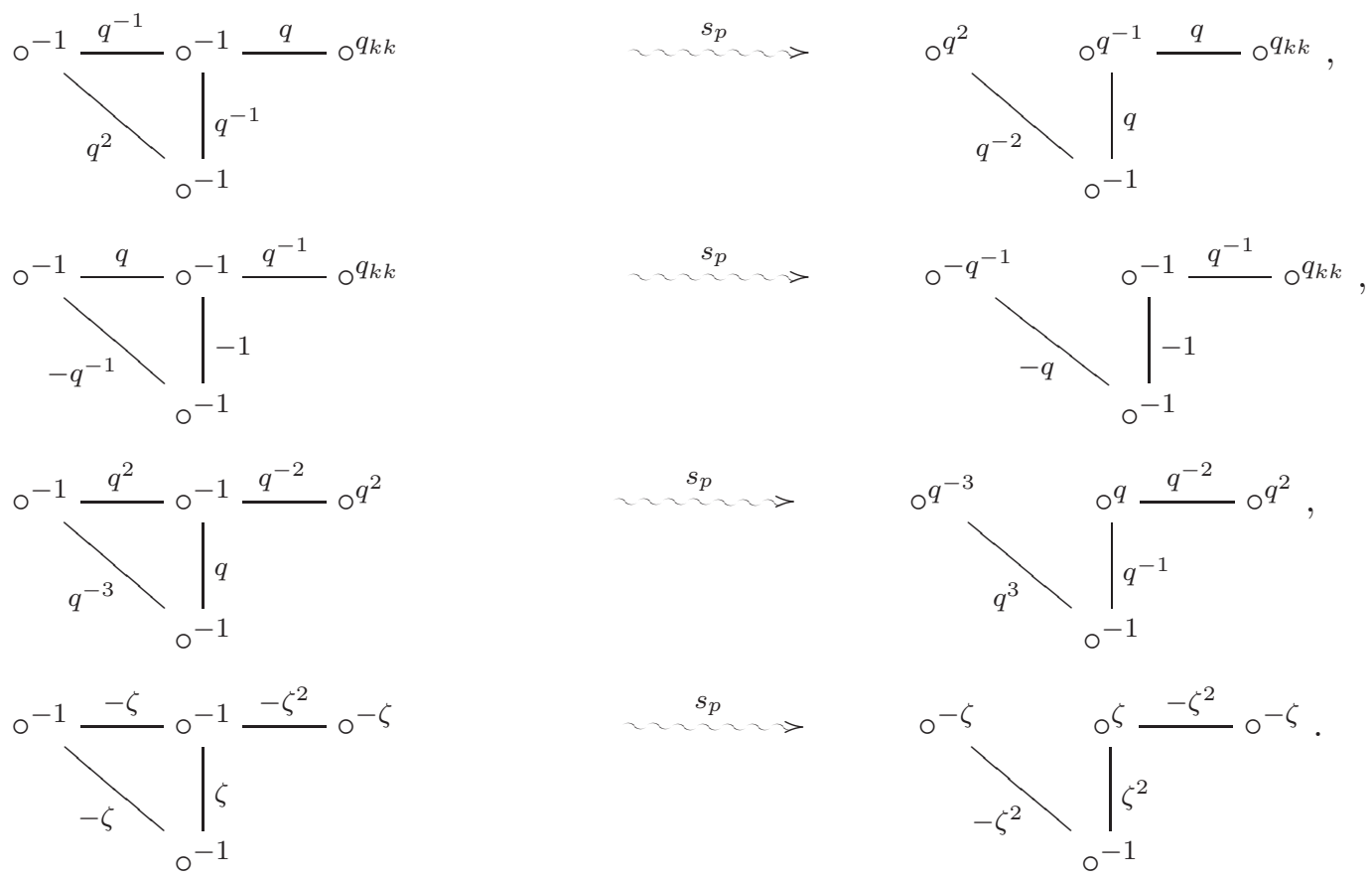

Note that $m_{p i}=m_{p j}=1$. If we fix the order $p<j<i<k$, and obtain that $T_{p}\left(\left[E_{j i}, E_{j k}\right]_{c}\right)=\left[\underline{E}_{p} \underline{E}_{j} \underline{E}_{p} \underline{E}_{i} \underline{E}_{p} \underline{E}_{j} \underline{E}_{k}\right]$. We can write $\underline{E}_{p} \underline{E}_{j} \underline{E}_{p} \underline{E}_{i} \underline{E}_{p} \underline{E}_{j} \underline{E}_{k}$ as a linear combination of greater words modulo $\mathcal{J}\left(s_{p}^{*} \chi\right)$ : in the first case, using (48); for the remaining cases, we use (59).

Lemma 3.13. Let $i, j \in\{1, \ldots, \theta\}$ be such that $q_{j j}=-1$, and also $q_{i i}= \pm \widetilde{q_{i j}} \in \mathbb{G}_{3}$, or $q_{i i} \widetilde{q_{i j}} \in \mathbb{G}_{6}$. Then, $T_{p}\left(\left[\left(E_{i i j}, E_{i j}\right]_{c}\right) \in \mathcal{J}\left(s_{p}^{*} \chi\right)\right.$, for any $p \in\{1, \ldots, \theta\}$. 
Proof. We denote $\mathbf{x}:=\left[\left(E_{i i j}, E_{i j}\right]_{c}\right.$. We begin with the case $p=j$. Note that $m_{p i}=1$ (because $q_{p p}=-1, \widetilde{q_{p i}} \neq 1$ ), $3 \alpha_{i}+2 \alpha_{p} \notin \Delta_{+}^{\chi}$, so

$$
s_{p}\left(3 \alpha_{i}+2 \alpha_{p}\right)=3 \alpha_{i}+\alpha_{p} \notin \Delta_{+}^{s_{p}^{*} \chi} .
$$

Using (71) and $\left(\operatorname{ad}_{\underline{c}} \underline{E}_{i}\right)^{3} \underline{E}_{p} \in \mathcal{J}\left(s_{p}^{*} \chi\right)$, we obtain that

$$
T_{p}(\mathbf{x})=a_{2} a_{1}\left[\left[\underline{E}_{p i}, \underline{E}_{i}\right]_{\underline{c}}, \underline{E}_{i}\right]_{\underline{c}} \in \mathcal{J}\left(s_{p}^{*} \chi\right) .
$$

Now let $p=i$. By Lemma 3.4 it is equivalent to prove that

$$
T_{p}\left(\mathbf{x}^{\prime}\right) \in \mathcal{J}\left(s_{p}^{*} \chi\right), \quad \text { where } \mathbf{x}^{\prime}:=\left[\underline{E}_{j p},\left[\underline{E}_{j p}, \underline{E}_{p}\right]_{c}\right]_{c},
$$

because we have proved that $T_{p}$ apply the generating relations of degree less than $\mathbf{x}$ in elements of $\mathcal{J}\left(s_{p}^{*} \chi\right)$. By (36),

$$
T_{p}\left(\mathbf{x}^{\prime}\right)=a_{1}^{2} a_{2} \quad\left[\left(\operatorname{ad}_{\underline{c}} \underline{E}_{p}\right) \underline{E}_{j}, \underline{E}_{j}\right]_{\underline{c}} \in \mathcal{J}\left(s_{p}^{*} \chi\right),
$$

because it holds that $\underline{q}_{j j}=-1$, or $\underline{q}_{j j} \underline{q}_{j p} \underline{q}_{p j}=1$.

Finally, let $p \neq i, j$; the case $m_{p i}=m_{p j}=0$ follows easily as in the previous Lemmata, so consider the case in which $p, i, j$ determine a connected subdiagram of rank three. We note that $q_{i i} \in \mathbb{G}_{3}$.

We take first $m_{p i} \neq 0, m_{p j}=0$. The possible braidings verify that $m_{p i}=1$, so for the order $p<i<j$,

$$
T_{p}(\mathbf{x})=\left[\left[\underline{E}_{p i},\left[\underline{E}_{p i}, \underline{E}_{j}\right]_{\underline{c}}\right]_{\underline{c}},\left[\underline{E}_{p i}, \underline{E}_{j}\right]_{\underline{c}}\right]_{\underline{c}}=\left[\underline{E}_{p} \underline{E}_{i}\left(\underline{E}_{p} \underline{E}_{i} \underline{E}_{j}\right)^{2}\right]_{\underline{c}},
$$

where we use that $\left(\operatorname{ad}_{c} \underline{E}_{p}\right) \underline{E}_{j}=0$ in $U\left(s_{p}^{*} \chi\right)$. As $q_{p i} q_{i p} q_{i i}=1$ or $q_{p i} q_{i p}= \pm q_{i i}$, we have that $\underline{q}_{i i}=-1$, or $\underline{q}_{i i} \underline{q}_{i p} \underline{q}_{p i}=1$, or $\underline{q}_{i i}^{2} \underline{q}_{i p} \underline{q}_{p i}=1$, or $\underline{q}_{i i}=-\underline{q}_{i p} \underline{q}_{p i} \in \mathbb{G}_{3}$, so $\underline{E}_{p} \underline{E}_{i} \underline{E}_{p} \underline{E}_{i} \underline{E}_{j} \underline{E}_{p} \underline{E}_{i} \underline{E}_{j}$ can be expressed as a linear combination of greater words modulo $\mathcal{J}\left(s_{p}^{*} \chi\right)$, using the quantum Serre relations or (45). We deduce that $T_{p}(\mathbf{x}) \in \mathcal{J}\left(s_{p}^{*} \chi\right)$, using the Lemma 3.4.

Now let $m_{p i}=0, m_{p j} \neq 0$. We note that $m_{p j}=1$ for any possible diagram, and in $U\left(s_{p}^{*} \chi\right)$ we have that:

$$
\begin{aligned}
T_{p}(\mathbf{x}) & =\left[\left(\operatorname{ad}_{\underline{c}} \underline{E}_{i}\right)^{2}\left(\operatorname{ad}_{\underline{c}} \underline{E}_{p}\right) \underline{E}_{j},\left(\operatorname{ad}_{\underline{c}} \underline{E}_{i}\right)\left(\operatorname{ad}_{\underline{c}} \underline{E}_{p}\right) \underline{E}_{j}\right]_{\underline{c}} \\
& =\underline{q}_{i p}^{3}\left[\left(\operatorname{ad}_{\underline{c}} \underline{E}_{p}\right)\left(\operatorname{ad}_{\underline{c}} \underline{E}_{i}\right)^{2} \underline{E}_{j},\left(\operatorname{ad}_{\underline{c}} \underline{E}_{p}\right)\left(\operatorname{ad}_{\underline{c}} \underline{E}_{i}\right) \underline{E}_{j}\right]_{\underline{c}} \\
& =\underline{q}_{i p}^{3}\left(\operatorname{ad}_{\underline{c}} \underline{E}_{p}\right)\left[\left(\operatorname{ad}_{\underline{c}} \underline{E}_{i}\right)^{2} \underline{E}_{j},\left(\operatorname{ad}_{\underline{c}} \underline{E}_{p}\right)\left(\operatorname{ad}_{\underline{c}} \underline{E}_{i}\right) \underline{E}_{j}\right]_{\underline{c}} \\
& =\underline{q}_{i p}^{3}\left(\operatorname{ad}_{\underline{c}} \underline{E}_{p}\right)\left[\left(\operatorname{ad}_{\underline{c}} \underline{E}_{i}\right)^{2}\left(\operatorname{ad}_{\underline{c}} \underline{E}_{j}\right) \underline{E}_{p},\left(\operatorname{ad}_{\underline{c}} \underline{E}_{i}\right) \underline{E}_{j}\right]_{\underline{c}}=0,
\end{aligned}
$$

by applying (69) (because $\left.\left(\operatorname{ad}_{\underline{c}} \underline{E}_{p}\right) \underline{E}_{i}=0\right)$, (9), and also that (46) is a generator of $\mathcal{J}\left(s_{p}^{*} \chi\right)$, because $\underline{q}_{i i}=q_{i i} \in \mathbb{G}_{3}, \underline{m}_{j i}=\underline{m}_{j p}=1$, and (45) is another generator by Lemma 3.8.

Finally, consider $m_{p i}, m_{p j} \neq 0$. There exists just one possible braiding: $q_{p p}=-1=$

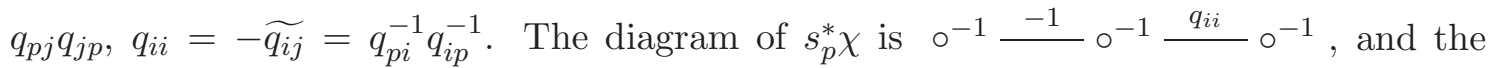
solution is analogous to the previous case, but now we use (48).

Lemma 3.14. Let $i, j, k \in\{1, \ldots, \theta\}$ be such that $q_{i i}= \pm \widetilde{q_{i j}} \in \mathbb{G}_{3}, \widetilde{q_{i k}}=1$, and $q_{j j} \widetilde{q_{i j}}=$ $q_{j j} \widetilde{q_{j k}}=1$ or $q_{j j}=-1, \widetilde{q_{i j}} \widetilde{q_{j k}}=1$. Then, for any $p \in\{1, \ldots, \theta\}$,

$$
T_{p}\left(\left[E_{i i j k}, E_{i j}\right]_{c}\right) \in \mathcal{J}\left(s_{p}^{*} \chi\right) .
$$


Proof. We denote $\mathbf{x}=\left[E_{i i j k}, E_{i j}\right]_{c}$. We begin with the case $p=k$. In all the cases we have that $m_{k j}=1$, and $s_{p}^{*} \chi$ satisfies the same conditions, so (46) is a generator of $\mathcal{J}\left(s_{p}^{*} \chi\right)$. Then,

$$
\begin{aligned}
T_{p}(\mathbf{x}) & \cong\left[\left(\operatorname{ad}_{\underline{c}} \underline{E}_{i}\right)^{2}\left(a_{1} \underline{E}_{j}\right),\left(\operatorname{ad}_{\underline{c}} \underline{E}_{i}\right)\left(\operatorname{ad}_{\underline{c}} \underline{E}_{k}\right) \underline{E}_{j}\right]_{\underline{c}} \cong a_{1} q_{i k}\left[\underline{E}_{i i j}, \underline{E}_{k i j}\right]_{\underline{c}} \\
& \cong a_{1} q_{i k}\left[\underline{E}_{i i j k}, \underline{E}_{i j}\right]_{\underline{c}} \cong 0 \quad\left(\bmod \mathcal{J}\left(s_{p}^{*} \chi\right)\right),
\end{aligned}
$$

where we apply first (71), then (69),$\left(\operatorname{ad}_{c b} \underline{E}_{k}\right) \underline{E}_{i}=0$ for the second line, y finally (9) plus the fact that (45) is a generator of $\mathcal{J}\left(s_{p}^{*} \chi\right)$. The cases $p=i, p=j$ are proved in a similar way to the case $p=i$ of previous Lemma.

Finally take $p \neq i, j, k$, and assume that $p$ is connected with at least one of the other vertices; in other case the proof is easy as above. We have two possible cases: $m_{p i}=1$, $m_{p j}=m_{p k}=0$, or $m_{p k}=1, m_{p j}=m_{p i}=0$. For the first one,

$$
T_{p}(\mathbf{x})=\left[\left[\underline{E}_{p i},\left[\underline{E}_{p i}, \underline{E}_{j k}\right]_{\underline{c}}\right]_{\underline{c}},\left[\underline{E}_{p i}, \underline{E}_{j}\right]_{\underline{c}}\right]_{\underline{c}},
$$

and we have two possibilities:

- if $q_{p p}=-1$, then $q_{i i} \widetilde{q_{i p}}=1$, and $\underline{q}_{i i}=-1$, so (48) is a generator of $\mathcal{J}\left(s_{p}^{*} \chi\right)$ for the subdiagram determined by $p, i, j$. Therefore $T_{p}(\mathbf{x}) \in \mathcal{J}\left(s_{p}^{*} \chi\right)$.

- if $q_{p p} \neq-1$, then $q_{p p}=q_{i p}^{-1} q_{p i}^{-1}=q_{i i}$, so $s_{p}^{*} \chi$ is twist equivalent to $\chi$ and (46) is a generator of $\mathcal{J}\left(s_{p}^{*} \chi\right)$. Then $T_{p}(\mathbf{x}) \in \mathcal{J}\left(s_{p}^{*} \chi\right)$, because it is obtained after to apply $\left(\operatorname{ad}_{c p} \underline{E}_{p}\right)^{3}$ to (46) and multiply by a non-zero scalar, where we use also the quantum Serre relations involving $\underline{E}_{p}$.

For the second case, we use $\left(\operatorname{ad}_{\underline{c}} \underline{E}_{p}\right) \underline{E}_{i},\left(\operatorname{ad}_{\underline{c}} \underline{E}_{p}\right) \underline{E}_{j} \in \mathcal{J}\left(s_{p}^{*} \chi\right)$ to obtain that

$$
\begin{aligned}
& T_{p}(\mathbf{x}) \cong\left[\left(\operatorname{ad}_{\underline{c}} \underline{E}_{i}\right)^{2}\left(\operatorname{ad}_{\underline{c}} \underline{E}_{j}\right)\left(\operatorname{ad}_{\underline{c}} \underline{E}_{p}\right) \underline{E}_{k},\left(\operatorname{ad}_{\underline{c}} \underline{E}_{i}\right) \underline{E}_{j}\right]_{\underline{c}} \\
& \cong \underline{q}_{i p}^{2} \underline{q}_{j p}\left[\left(\operatorname{ad}_{\underline{c}} \underline{E}_{p}\right)\left(\operatorname{ad}_{\underline{c}} \underline{E}_{i}\right)^{2}\left(\operatorname{ad}_{\underline{c}} \underline{E}_{j}\right) \underline{E}_{k},\left(\operatorname{ad}_{\underline{c}} \underline{E}_{i}\right) \underline{E}_{j}\right]_{\underline{c}} \\
& \cong \underline{q}_{i p}^{2} \underline{q}_{j p}\left(\operatorname{ad}_{\underline{c}} \underline{E}_{p}\right)\left(\left[\left(\operatorname{ad}_{\underline{c}} \underline{E}_{i}\right)^{2}\left(\operatorname{ad}_{\underline{c}} \underline{E}_{j}\right) \underline{E}_{k},\left(\operatorname{ad}_{\underline{c}} \underline{E}_{i}\right) \underline{E}_{j}\right]_{\underline{c}}\right) \cong 0 \quad\left(\bmod \mathcal{J}\left(s_{p}^{*} \chi\right)\right),
\end{aligned}
$$

by (69) and the fact that (46) is a generator of $\mathcal{J}\left(s_{p}^{*} \chi\right)$.

Lemma 3.15. Let $i, j, k \in\{1, \ldots, \theta\}$ be such that $\widetilde{q_{i k}}, \widetilde{q_{i j}}, \widetilde{q_{j k}} \neq 1$. Then, for any $p$,

$$
T_{p}\left(E_{i j k}-\frac{1-\widetilde{q_{j k}}}{q_{k j}\left(1-\widetilde{q_{i k}}\right)}\left[E_{i k}, E_{j}\right]_{c}-q_{i j}\left(1-\widetilde{q_{j k}}\right) E_{j} E_{i k}\right) \in \mathcal{J}\left(s_{p}^{*} \chi\right) \text {. }
$$

Proof. Let $\mathbf{x}=E_{i j k}-\frac{1-\widetilde{q_{j k}}}{q_{k j}\left(1-\widetilde{q_{i k}}\right)}\left[E_{i k}, E_{j}\right]_{c}-q_{i j}\left(1-\widetilde{q_{j k}}\right) E_{j} E_{i k}$. By a direct computation we obtain the same relation, up to an scalar, if we permute the vertices $i, j, k$, where we use that $\widetilde{q_{i k}} \widetilde{q_{i j}} \widetilde{q_{j k}}=1$, so it is enough to consider one of these permutations for each $p$.

Consider then $p=k$, which is analogous to take $p=i$ or $p=j$. Note that $\left\{m_{p i}, m_{p j}\right\}=$ $\{1,1\}$, or $\left\{m_{p i}, m_{p j}\right\}=\{1,2\}$, so we fix $m_{p j}=1, m_{p i} \in\{1,2\}$. By (71),

$$
\begin{aligned}
T_{p}\left(E_{i j k}\right) & =\left(q_{p p}^{-1} q_{p j}^{-1} q_{j p}^{-1}-1\right) q_{j p} q_{p p}\left[\left(\operatorname{ad}_{\underline{c}} \underline{E}_{p}\right)^{m_{p i}} \underline{E}_{i}, \underline{E}_{j}\right]_{\underline{c}}, \\
T_{p}\left(\left[E_{i k}, E_{j}\right]_{c}\right) & =\left(q_{p p}^{-1-m_{p i}} q_{p i}^{-1} q_{i p}^{-1}-1\right) q_{i p} q_{p p}\left[\left(\operatorname{ad}_{\underline{c}} \underline{E}_{p}\right)^{m_{p i}-1} \underline{E}_{i},\left(\operatorname{ad}_{\underline{c}} \underline{E}_{p}\right) \underline{E}_{j}\right]_{\underline{c}}, \\
T_{p}\left(E_{j} E_{i k}\right) & =\left(q_{p p}^{-1-m_{p i}} q_{p i}^{-1} q_{i p}^{-1}-1\right) q_{i p} q_{p p}\left(\operatorname{ad}_{\underline{c}} \underline{E}_{p}\right) \underline{E}_{j}\left(\operatorname{ad}_{\underline{c}} \underline{E}_{p}\right)^{m_{p i}-1} \underline{E}_{i} .
\end{aligned}
$$

If $m_{p i}=2$, or $m_{p i}=1, q_{p p} \neq-1$, then $\underline{q}_{i k} \underline{q}_{k i}, \underline{q}_{i j} \underline{q}_{j i}, \underline{q}_{j k} \underline{q}_{k j} \neq 1$ and we deduce that $T_{p}(\mathbf{x}) \in \mathcal{J}\left(s_{p}^{*} \chi\right)$ from the fact that (47) is a generator of $\mathcal{J}\left(s_{p}^{*} \chi\right)$, because we can write then $\underline{E}_{p}^{m_{p i}} \underline{E}_{i} \underline{E}_{j}$ as a linear combination of greater words (for the order on the letters 
$p<i<j$ ), modulo $\mathcal{J}\left(s_{p}^{*} \chi\right)$, y apply then Lemma 3.4. If $q_{p p}=-1$ then $\underline{q}_{i j} \underline{q}_{j i}=1$, so $\left(\operatorname{ad}_{\underline{c}} \underline{E}_{i}\right) \underline{E}_{j} \in \mathcal{J}\left(s_{p}^{*} \chi\right)$. By a direct computation, there exists $a \in \mathbf{k}^{\times}$such that

$$
T_{p}(\mathbf{x})=a\left(\operatorname{ad}_{\underline{c}} \underline{E}_{p}\right)\left(\operatorname{ad}_{\underline{c}} \underline{E}_{i}\right) \underline{E}_{j} \in \mathcal{J}\left(s_{p}^{*} \chi\right) .
$$

Let $p \neq i, j, k$. We note that $p$ is not connected with any of the other vertices (so the proof follows easily as in the previous Lemmata), or $p$ is connected just with one of these vertices. For the last case we can assume that $m_{p i} \neq 0$, so the unique possibility is $m_{p i}=m_{i p}=1$. Then $\widetilde{q_{i k}}=\underline{q}_{i k} \underline{q}_{k i}, \widetilde{q_{i j}}=\underline{q}_{i j} \underline{q}_{j i}, \widetilde{q_{j k}}=\underline{q}_{k j} \underline{q}_{j k} \neq 1$, so (47) is a generator of $\mathcal{J}\left(s_{p}^{*} \chi\right)$. By Lemma 3.7 and the relations $\left(\operatorname{ad}_{\underline{c}} \underline{E}_{p}\right) \underline{E}_{j}=\left(\operatorname{ad}_{\underline{c}} \underline{E}_{p}\right) \underline{E}_{k}=0$, we deduce that $T_{p}(\mathbf{x})$ is obtained, up to a non-zero scalar, after to apply $\left(\operatorname{ad}_{\underline{c}} \underline{E}_{p}\right)$ to (47), modulo $\mathcal{J}\left(s_{p}^{*} \chi\right)$, so $T_{p}(\mathbf{x}) \in \mathcal{J}\left(s_{p}^{*} \chi\right)$.

Lemma 3.16. Let $i, j, k \in\{1, \ldots, \theta\}$ be such that

(i) $q_{i i}=q_{j j}=-1,{\widetilde{q_{i j}}}^{2}={\widetilde{q_{j k}}}^{-1}, \widetilde{q_{i k}}=1$, or

(ii) $\widetilde{q_{i j}}=q_{j j}=-1, q_{i i}=-{\widetilde{q_{j k}}}^{2} \in \mathbb{G}_{3}, \widetilde{q_{i k}}=1$, or

(iii) $q_{k k}=\widetilde{q_{j k}}=q_{j j}=-1, q_{i i}=-\widetilde{q_{i j}} \in \mathbb{G}_{3}, \widetilde{q_{i k}}=1$, or

(iv) $q_{j j}=-1, \widetilde{q_{i j}}=q_{i i}^{-2}, \widetilde{q_{j k}}=-q_{i i}^{-3}, \widetilde{q_{i k}}=1$, or

(v) $q_{i i}=q_{j j}=q_{k k}=-1, \pm \widetilde{q_{i j}}=\widetilde{q_{j k}} \in \mathbb{G}_{3}, \widetilde{q_{i k}}=1$,

Then, for any $p, T_{p}\left(\left[\left[E_{i j}, E_{i j k}\right]_{c}, E_{j}\right]_{c}\right) \in \mathcal{J}\left(s_{p}^{*} \chi\right)$.

Proof. Denote $\mathbf{x}=\left[\left[E_{i j}, E_{i j k}\right]_{c}, E_{j}\right]_{c}$; we analyze each case.

(i) We begin with the case $p=k$; by (71) and as $\underline{E}_{i}^{2}, \underline{E}_{j}^{2},\left(\operatorname{ad}_{\underline{c}} \underline{E}_{i}\right) \underline{E}_{p}$ are generators of $\mathcal{J}\left(s_{p}^{*} \chi\right)$ (note that $s_{p}^{*} \chi$ is twist equivalent to $\chi$ ), we have that

$$
\begin{aligned}
T_{p}(\mathbf{x}) & \cong a_{1}\left[\left[\underline{E}_{i p j}, \underline{E}_{i j}\right]_{\underline{c}}, \underline{E}_{p j}\right]_{\underline{c}} \cong a_{1} \underline{q}_{i p}\left[\left[\underline{E}_{p i j}, \underline{E}_{i j}\right]_{\underline{c}}, \underline{E}_{p j}\right]_{\underline{c}} \\
& \cong a\left[\left[\underline{E}_{p j i}, \underline{E}_{j i}\right]_{\underline{c}}, \underline{E}_{p j}\right]_{\underline{c}} \cong a\left[\underline{E}_{p} \underline{E}_{j} \underline{E}_{i} \underline{E}_{j} \underline{E}_{i} \underline{E}_{p} \underline{E}_{j}\right]_{\underline{c}}\left(\bmod \mathcal{J}\left(s_{p}^{*} \chi\right)\right),
\end{aligned}
$$

for some $a \in \mathbf{k}^{\times}$, where we use the order on the letters $p<j<i$. As (48) is also a generator of $\mathcal{J}\left(s_{p}^{*} \chi\right)$, we can write $\underline{E}_{j} \underline{E}_{i} \underline{E}_{j} \underline{E}_{i} \underline{E}_{p} \underline{E}_{j}$ as a linear combination of other words, greater than this word or beginning with $\underline{E}_{p}$. Multiplying on the left by $\underline{E}_{p}$ and using the quantum Serre relations $\underline{E}_{p} \underline{E}_{j} \underline{E}_{i} \underline{E}_{j} \underline{E}_{i} \underline{E}_{p} \underline{E}_{j}$ is expressed as a linear combination of greater words modulo $\mathcal{J}\left(s_{p}^{*} \chi\right)$, so by Lemma 3.4. $T_{p}(\mathbf{x}) \in \mathcal{J}\left(s_{p}^{*} \chi\right)$.

Let $p=j$; note that $m_{p i}=m_{p k}=1$. Also, $\underline{q}_{i i}^{-1}=\underline{q}_{i k} \underline{q}_{k i}$, so $\left(\operatorname{ad}_{\underline{c}} \underline{E}_{i}\right)^{2} \underline{E}_{k} \in \mathcal{J}\left(s_{p}^{*} \chi\right)$; use (71) and work as in the case $p=i$ of Lemma 3.13 to obtain that

$$
T_{p}(\mathbf{x})=a_{1}^{2}\left[\underline{E}_{i}, \underline{E}_{i p k}\right]_{\underline{c}} \underline{F}_{p} \underline{L}_{p}^{-1}-a_{1}^{2} q_{i p}^{2} q_{k p} \underline{F}_{p} \underline{L}_{p}^{-1}\left[\underline{E}_{i}, \underline{E}_{i p k}\right]_{\underline{c}}=b\left(\operatorname{ad}_{\underline{c}} \underline{E}_{i}\right)^{2} \underline{E}_{k} \in \mathcal{J}\left(s_{p}^{*} \chi\right),
$$

for some $b \in \mathbf{k}^{\times}$.

Let now $p=i$. As in the previous Lemmata, it is enough to prove the statement for

$$
\mathbf{x}^{\prime}:=\left[\left[E_{k j p}, E_{j p}\right]_{c}, E_{j}\right]_{c} .
$$

We apply (71) to obtain, for the order on the letters $k<i<j$,

$$
T_{p}\left(\mathbf{x}^{\prime}\right)=\left[\left[\left[\underline{E}_{k}, a_{1} \underline{E}_{j}\right]_{\underline{c}}, \underline{E}_{j}\right]_{\underline{c}},\left(\operatorname{ad}_{\underline{c}} \underline{E}_{p}\right) \underline{E}_{j}\right]_{\underline{c}}=a_{1}^{2}\left[\underline{E}_{k} \underline{E}_{j}^{2} \underline{E}_{p} \underline{E}_{j}\right]_{\underline{c}} .
$$

As $\underline{q}_{j j} \underline{q}_{j i} \underline{q}_{i j}=\underline{q}_{j j}^{2} \underline{q}_{j k} \underline{q}_{k j}=1$, we deduce by (50) if $\underline{q}_{j j} \in \mathbb{G}_{3}$, or by $\left(\operatorname{ad}_{\underline{c}} \underline{E}_{j}\right)^{2} \underline{E}_{p}=$ $\left(\operatorname{ad}_{\underline{c}} \underline{E}_{j}\right)^{3} \underline{E}_{k}=0$, if $\underline{q}_{j j} \notin \mathbb{G}_{3}$, that $\underline{E}_{k} \underline{E}_{j}^{2} \underline{E}_{p} \underline{E}_{j}$ is a linear combination of greater words, so $T_{p}\left(\mathbf{x}^{\prime}\right) \in \mathcal{J}\left(s_{p}^{*} \chi\right)$, and then $T_{p}(\mathbf{x}) \in \mathcal{J}\left(s_{p}^{*} \chi\right)$. 
If $p \neq i, j, k$, then $p$ is not connected with these three vertices, or $p$ is connected just with $i$, or $p$ is connected with two vertices. For the second case we have that $q_{p p} \widetilde{q_{p i}}=1$, or $q_{p p}=-1, \widetilde{q_{i p}} \widetilde{q_{i j}}=1$, so

$$
\begin{aligned}
T_{p}(\mathbf{x}) & \left.\cong\left[\underline{E}_{p i j}, \underline{E}_{p i j k}\right]_{\underline{c}}, \underline{E}_{j}\right]_{\underline{c}} \cong\left(\operatorname{ad}_{\underline{c}} \underline{E}_{p}\right)\left(\left[\left[\underline{E}_{p i j}, \underline{E}_{i j k}\right]_{\underline{c}}, \underline{E}_{j}\right]_{\underline{c}}\right) \\
& \cong\left(\operatorname{ad}_{\underline{c}} \underline{E}_{p}\right)\left(\left[\underline{E}_{p} \underline{E}_{i} \underline{E}_{j} \underline{E}_{i} \underline{E}_{j} \underline{E}_{k} \underline{E}_{j}\right]_{\underline{c}}\right) \bmod \mathcal{J}\left(s_{p}^{*} \chi\right)
\end{aligned}
$$

by using first Lemma 3.7, then $\left(\operatorname{ad}_{\underline{c}} \underline{E}_{p}\right) \underline{E}_{j},\left(\operatorname{ad}_{\underline{c}} \underline{E}_{p}\right) \underline{E}_{j},\left(\operatorname{ad}_{\underline{c}} \underline{E}_{p}\right) \underline{E}_{j} \in \mathcal{J}\left(s_{p}^{*} \chi\right)$, and fixing the order $p<i<j<k$. We conclude that $T_{p}(\mathbf{x}) \in \mathcal{J}\left(s_{p}^{*} \chi\right)$ by using (56) if $q_{p p}=-1$, or using the quantum Serre relations corresponding to $\operatorname{ad}_{\underline{c}} \underline{E}_{p}$ to write $\underline{E}_{p} \underline{E}_{i} \underline{E}_{j} \underline{E}_{i} \underline{E}_{j} \underline{E}_{k} \underline{E}_{j}$ as a linear combination of greater words and apply Lemma 3.4 to deduce that

$$
\left[\underline{E}_{p} \underline{E}_{i} \underline{E}_{j} \underline{E}_{i} \underline{E}_{j} \underline{E}_{k} \underline{E}_{j}\right]_{\underline{c}} \in \mathcal{J}\left(s_{p}^{*} \chi\right) \text {. }
$$

For the last case, we have two possibilities:

- $p$ is connected with $i$ and $j$, in which case the proof follows by the fact that (58) is a generator of the ideal, or

- $p$ is connected with $j$ and $k$, in which case it follows because (57) is a generator of $\mathcal{J}\left(s_{p}^{*} \chi\right)$.

(ii), (iii), (iv), (v) If $p \in\{i, j, k\}$ the proof is completely analogous to the previous case.

Let $p \neq i, j, k$, so $p$ is not connected with any of these vertices, or it connected only with $i$, or only with $k$. The first case is easy. For the second case, $m_{p i}=1$, because $q_{p p}=1$ or $q_{p p}^{-1}=\widetilde{q_{i p}} \neq-1$, and the solution follows as in the previous case. For the last case, $m_{p k}=1$ and the proof is also analogous, considering the previous $\mathbf{x}^{\prime}$.

Lemma 3.17. (i) Let $i, j, k, l \in\{1, \ldots, \theta\}$ be such that $q_{k k}=-1,{\widetilde{q_{j k}}}^{2}={\widetilde{q_{l k}}}^{-1}=q_{l l}$, $q_{j j} \widetilde{q_{i j}}=q_{j j} \widetilde{q_{j k}}=\widetilde{q_{i k}}=\widetilde{q_{i l}}=\widetilde{q_{j l}}=1$. Then, for all $p, T_{p}\left(\left[\left[\left[E_{i j k l}, E_{k}\right]_{c}, E_{j}\right]_{c}, E_{k}\right]_{c}\right) \in$ $\mathcal{J}\left(s_{p}^{*} \chi\right)$.

(ii) Let $i, j, k \in\{1, \ldots, \theta\}$ be such that $q_{i i}=q_{j j}=-1,{\widetilde{q_{i j}}}^{3}={\widetilde{q_{j k}}}^{-1}=q_{k k} \neq \pm 1, \widetilde{q_{i k}}=1$. Then, for all $p, T_{p}\left(\left[\left[E_{i j},\left[E_{i j}, E_{i j k}\right]_{c}\right]_{c}, E_{j}\right]_{c}\right) \in \mathcal{J}\left(s_{p}^{*} \chi\right)$.

Proof. (i) The proof is analogous to (i) of the previous Lemma, because if $p \neq i, j, k, l$ is connected with some of them, then $p$ is connected only with $i$ with the same conditions.

(ii) If $p \in\{i, j, k\}$ the proof is completely analogous to the previous Lemma. If $p \neq i, j, k$ is connected with some of them, then $p$ is connected only with $i$ and $q_{p p}=-1, \widetilde{q_{p i}}=-\widetilde{q_{i j}} \in$ $\mathbb{G}_{4}$. Anyway, the proof is analogous to the previous Lemma.

Lemma 3.18. (i) Let $i, j, k, l \in\{1, \ldots, \theta\}$ be such that $q_{l l}={\widetilde{q_{l k}}}^{-1}=q_{k k}={\widetilde{q_{j k}}}^{-1}=q^{2}$, $\widetilde{q_{i j}}=q_{i i}^{-1}=q^{3}$ for some $q \in \mathbf{k}^{\times}, q_{j j}=-1, \widetilde{q_{i k}}=\widetilde{q_{i l}}=\widetilde{q_{j l}}=1$. Then, for all $p$, $T_{p}\left(\left[\left[\left[E_{i j k}, E_{j}\right]_{c},\left[E_{i j k l}, E_{j}\right]_{c}\right]_{c}, E_{j k}\right]_{c}\right) \in \mathcal{J}\left(s_{p}^{*} \chi\right)$.

(ii) Let $i, j, k, l \in\{1, \ldots, \theta\}$ be such that $\widetilde{q_{j k}}=\widetilde{q_{i j}}=q_{j j}^{-1} \in \mathbb{G}_{4}^{\prime} \cup \mathbb{G}_{6}^{\prime}, q_{i i}=q_{k k}=-1$, $\widetilde{q_{i k}}=\widetilde{q_{i l}}=\widetilde{q_{j l}}=1,{\widetilde{q_{j k}}}^{3}=\widetilde{q_{l k}}$. Then, for all $p, T_{p}\left(\left[\left[E_{i j k},\left[E_{i j k l}, E_{k}\right]_{c}\right]_{c}, E_{j k}\right]_{c}\right) \in \mathcal{J}\left(s_{p}^{*} \chi\right)$.

Proof. (i) Let $\mathbf{x}=\left[\left[E_{i j k},\left[E_{i j k l}, E_{k}\right]_{c}\right]_{c}, E_{j k}\right]_{c}$. If $p \notin\{i, j, k, l\}$, then $p$ is not connected with $i, j, k, l$, so we consider the case $p \in\{i, j, k, l\}$. If $p=i$, the diagram for $s_{p}^{*} \chi$ is the same, and $T_{p}(\mathbf{x})$ corresponds to a relation of degree $3 \alpha_{p}+5 \alpha_{j}+3 \alpha_{k}+\alpha_{l}$, obtained after 
to apply $\operatorname{ad}_{c} \underline{E}_{p}$ to (58). Similar situations hold when $p=k$ and $p=l$. If $p=j$, then $T_{p}(\mathbf{x})$ corresponds to the relation (48), and the proof follows.

(ii) The proof is very similar to previous item, because $p \in\{i, j, k, l\}$, or $p$ is not connected with $i, j, k, l$.

Lemma 3.19. Let $i, j, k, l \in\{1, \ldots, \theta\}$ be such that one of the following situations hold:

$$
\begin{aligned}
& \circ q_{k k}=-1, q_{i i}=\widetilde{q_{i j}}-1=q_{j j}^{2}, \widetilde{q_{k l}}=q_{l l}^{-1}=q_{j j}^{3}, \widetilde{q_{j k}}=q_{j j}^{-1}, \widetilde{q_{i k}}=\widetilde{q_{i l}}=\widetilde{q_{j l}}=1, \\
& \circ q_{i i}=\widetilde{q_{i j}}-1=-q_{l l}^{-1}=-\widetilde{q_{k l}}, q_{j j}=\widetilde{q_{j k}}=q_{k k}=-1, \widetilde{q_{i k}}=\widetilde{q_{i l}}=\widetilde{q_{j l}}=1 \text {, or } \\
& \circ q_{j j}=\widetilde{q_{j k}}-1 \in \mathbb{G}_{3}, q_{i i}=\widetilde{q_{i j}}-1=q_{l l}=\widetilde{q_{k l}}-1=-q_{j j}, q_{k k}=-1, \widetilde{q_{i k}}=\widetilde{q_{i l}}=\widetilde{q_{j l}}=1 .
\end{aligned}
$$

Then, $T_{p}\left(\left[\left[E_{i j k l}, E_{j}\right]_{c}, E_{k}\right]_{c}-q_{j k}\left({\widetilde{q_{i j}}}^{-1}-q_{j j}\right)\left[\left[E_{i j k l}, E_{k}\right]_{c}, E_{j}\right]_{c}\right) \in \mathcal{J}\left(s_{p}^{*} \chi\right)$, for all $p$.

Proof. No one of these diagrams can be extended in order to have a connected diagram with finite root system. In consequence, it is enough to consider (as in the previous Lemmata) the cases $p \in\{i, j, k, l\}$. The proof for these cases is similar to the Lemma 3.15, up to consider the necessary relations under the conditions of these new situations.

Lemma 3.20. (i) Let $i, j, k \in\{1, \ldots, \theta\}$ be such that $q_{k k}=q_{j j}={\widetilde{q_{i j}}}^{-1}={\widetilde{q_{j k}}}^{-1} \in \mathbb{G}_{9}$, $\widetilde{q_{i k}}=1, q_{i i}=q_{k k}^{6}$. Then, for all $p, T_{p}\left(\left[\left[E_{i i j}, E_{i i j k}\right]_{c}, E_{i j}\right]_{c}\right) \in \mathcal{J}\left(s_{p}^{*} \chi\right)$.

(ii) Let $i, j, k \in\{1, \ldots, \theta\}$ be such that $q_{i i}={\widetilde{q_{i j}}}^{-1} \in \mathbb{G}_{9}, q_{j j}={\widetilde{q_{j k}}}^{-1}=q_{i i}^{5}, \widetilde{q_{i k}}=1, q_{k k}=$ $q_{i i}^{6}$. Then, for all $p, T_{p}\left(\left[\left[E_{i j k}, E_{j}\right]_{c}, E_{k}\right]_{c}-\left(1+\widetilde{q_{j k}}\right)^{-1} q_{j k}\left[\left[E_{i j k}, E_{k}\right]_{c}, E_{j}\right]_{c}\right) \in \mathcal{J}\left(s_{p}^{*} \chi\right)$.

Proof. For both cases, there exist no extensions of these diagrams. In consequence, it is enough to consider the case $p \in\{i, j, k\}$.

(i) The cases $p=k$ and $p=j$ follows easily because the diagram for $s_{p}^{*} \chi$ in these cases coincide with the one for $\chi$.

If $p=i$, then $T_{p}\left(\left[\left[E_{p p j}, E_{p p j k}\right]_{c}, E_{p j}\right]_{c}\right)$ is, up to an scalar, $\left[\underline{E}_{p j k}, \underline{E}_{j}\right]_{\underline{c}}$, which belongs to $\mathcal{J}\left(s_{p}^{*} \chi\right)$ because $\left(\operatorname{ad}_{\underline{c}} \underline{E}_{j}\right)^{2} \underline{E}_{p},\left(\operatorname{ad}_{\underline{c}} \underline{E}_{j}\right)^{2} \underline{E}_{k} \in \mathcal{J}\left(s_{p}^{*} \chi\right)$ (we have that $\left.\underline{q}_{j j} \widetilde{\widetilde{q}} \underline{q}_{j k}=\underline{q}_{j j} \underline{q}_{j p}=1\right)$.

(ii) The proof is similar to the one for Lemma 3.15 ,

Lemma 3.21. (i) Let $i, j, k \in\{1, \ldots, \theta\}$ be such that $q_{j j}={\widetilde{q_{i j}}}^{2}=\widetilde{q_{j k}} \in \mathbb{G}_{3}, \widetilde{q_{i k}}=1$. Then, for all $p, T_{p}\left(\left[\left[E_{i j k}, E_{j}\right]_{c}, E_{j}\right]_{c}\right) \in \mathcal{J}\left(s_{p}^{*} \chi\right)$.

(ii) Let $i, j, k \in\{1, \ldots, \theta\}$ be such that $q_{j j}={\widetilde{q_{i j}}}^{3}=\widetilde{q_{j k}} \in \mathbb{G}_{4}, \widetilde{q_{i k}}=1$. Then, for all $p$, $T_{p}\left(\left[\left[\left[E_{i j k}, E_{j}\right]_{c}, E_{j}\right]_{c}, E_{j}\right]_{c}\right) \in \mathcal{J}\left(s_{p}^{*} \chi\right)$.

Proof. (i) Let $\mathbf{x}=\left[\left[E_{i j k}, E_{j}\right]_{c}, E_{j}\right]_{c}$. For the case $p=k$, note that $m_{p j}=1$ in all the cases, so for the order $i<p<j$ on the letters we have by (71):

$$
T_{p}(\mathbf{x})=a_{1}\left[\left[\underline{E}_{i j}, \underline{E}_{p j}\right]_{\underline{c}}, \underline{E}_{p j}\right]_{\underline{c}}=\left[\underline{E}_{i} \underline{E}_{j} \underline{E}_{p} \underline{E}_{j} \underline{E}_{p} \underline{E}_{j}\right]_{\underline{c}} .
$$

As $\left(\operatorname{ad}_{\underline{c}} \underline{E}_{p}\right)^{2} \underline{E}_{j} \in \mathcal{J}\left(s_{p}^{*} \chi\right)$, or (48) is a generator of $\mathcal{J}\left(s_{p}^{*} \chi\right)$, and also $\underline{E}_{j}^{3},\left(\operatorname{ad}_{\underline{c}} \underline{E}_{k}\right) \underline{E}_{i} \in$ $\mathcal{J}\left(s_{p}^{*} \chi\right), \underline{E}_{i} \underline{E}_{j} \underline{E}_{k} \underline{E}_{j} \underline{E}_{k} \underline{E}_{j}$ can be written as a linear combination of greater words modulo $\mathcal{J}\left(s_{p}^{*} \chi\right)$, so $T_{p}(\mathbf{x}) \in \mathcal{J}\left(s_{p}^{*} \chi\right)$.

Consider now $p=j$. By (71) and the relations defining $\mathcal{J}\left(s_{p}^{*} \chi\right)$,

$$
T_{p}(\mathbf{x})=\left[\left[\left[\underline{E}_{i},\left(\operatorname{ad}_{\underline{c}} \underline{E}_{p}\right)^{2} \underline{E}_{k}\right]_{\underline{c}}, \underline{F}_{p} \underline{L}_{p}^{-1}\right]_{\underline{c}}, \underline{F}_{p} \underline{L}_{p}^{-1}\right]_{\underline{c}}=a\left(\operatorname{ad}_{\underline{c}} \underline{E}_{i}\right) \underline{E}_{k},
$$


for some $a \in \mathbf{k}^{\times}$, so $T_{p}(\mathbf{x}) \in \mathcal{J}\left(s_{p}^{*} \chi\right)$.

Let $p=i$. It is equivalent to prove that $T_{p}\left(\mathbf{x}^{\prime}\right) \in \mathcal{J}\left(s_{p}^{*} \chi\right)$, where $\mathbf{x}^{\prime}=\left[\left[E_{k j p}, E_{j}\right]_{c}, E_{j}\right]_{c}$. We note that $m_{i j}=1$ for all the possible diagrams, so $T_{p}(\mathbf{x})=a_{1}\left[\left[\underline{E}_{k j}, \underline{E}_{p j}\right]_{\underline{c}}, \underline{E}_{p j}\right]_{\underline{c}}$, and a proof similar to the case $p=k$ tells us that $T_{p}\left(\mathbf{x}^{\prime}\right) \in \mathcal{J}\left(s_{p}^{*} \chi\right)$, so $T_{p}(\mathbf{x}) \in \mathcal{J}\left(s_{p}^{*} \chi\right)$.

Finally, if $p \neq i, j, k$, then $p$ is not connected to any of these vertices, or $p$ is connected only with $i$, or it is connected only with $k$. The proof of the first case is again trivial, and for the other two cases $T_{p}(\mathbf{x}) \in \mathcal{J}\left(s_{p}^{*} \chi\right)$, using Lemma 3.7 and the fact that $s_{p}^{*} \chi$ is twist equivalent to $\chi$.

(ii) The proof is analogous to (i) .

Lemma 3.22. (i) Let $i, j, k \in\{1, \ldots, \theta\}$ be such that $q_{i i}=\widetilde{q_{i j}}=-1, q_{j j}={\widetilde{q_{j k}}}^{-1} \neq-1$, $\widetilde{q_{i k}}=1$. Then, for all $p, T_{p}\left(\left[E_{i j}, E_{i j k}\right]_{c}\right) \in \mathcal{J}\left(s_{p}^{*} \chi\right)$.

(ii) Let $i, j, k \in\{1, \ldots, \theta\}$ be such that $q_{j j}=q_{k k}=\widetilde{q_{j k}}=-1, q_{i i}=-\widetilde{q_{i j}} \in \mathbb{G}_{3}, \widetilde{q_{i k}}=1$. Then, for all $p, T_{p}\left(\left[E_{i i j k}, E_{i j k}\right]_{c}\right) \in \mathcal{J}\left(s_{p}^{*} \chi\right)$.

Proof. (i) Let $\mathbf{x}=\left[E_{i j}, E_{i j k}\right]_{c}$. If $p=k, m_{p j}=1$ in all the possible diagrams and then:

$$
T_{p}(\mathbf{x})=a_{1}\left[\underline{E}_{i k j}, \underline{E}_{i j}\right]_{\underline{c}}=a_{1} \underline{q}_{i p}\left[\underline{E}_{k i j}, \underline{E}_{i j}\right]_{\underline{\underline{c}}} .
$$

We consider the two possible values of $q_{p p}$. When $q_{p p} \neq-1$, the diagram is of Cartan $C_{3}$ type, associated to a root of order 4 , and $s_{p}^{*} \chi$ is twist equivalent to $\chi$. Therefore $T_{p}(\mathbf{x}) \in \mathcal{J}\left(s_{p}^{*} \chi\right)$, using that (54) is again a generator of $\mathcal{J}\left(s_{p}^{*} \chi\right)$. If $q_{p p}=-1$, then $q_{j j} \in \mathbb{G}_{3} \cup \mathbb{G}_{4} \cup \mathbb{G}_{6}$, and $\underline{q}_{j j}=\underline{q}_{i j}=\underline{q}_{i i}=-1$, so (43) is a generator of $\mathcal{J}\left(s_{p}^{*} \chi\right)$ and

$$
T_{p}(\mathbf{x})=a_{1} \underline{q}_{i p}\left[\underline{E}_{k i j}, \underline{E}_{i j}\right]_{\underline{c}}=a_{1} \underline{q}_{i p} \operatorname{ad}_{\underline{c}} \underline{E}_{k}\left(E_{i j}^{2}\right) \in \mathcal{J}\left(s_{p}^{*} \chi\right) .
$$

If $p=j$, we consider the different possible orders of $q_{p p}$. If $q_{p p} \in \mathbb{G}_{4} \cup \mathbb{G}_{6}$, then $p$ is a Cartan vertex and $s_{p}^{*} \chi$ is twist equivalent to $s_{p}^{*} \chi$, and $m_{p i}=2,3$

$$
T_{p}(\mathbf{x})=a_{m_{p i}}\left[\left(\operatorname{ad}_{\underline{c}} \underline{E}_{p}\right)^{m_{p i}-1} \underline{E}_{i},\left[\left(\operatorname{ad}_{\underline{c}} \underline{E}_{p}\right)^{m_{p i}-1} \underline{E}_{i}, \underline{E}_{p k}\right]_{\underline{c}}\right]_{\underline{c}}=\left[\underline{E}_{p}^{m_{p i-1}} \underline{E}_{i} \underline{E}_{p}^{m_{p i-1}} \underline{E}_{i} \underline{E}_{p} \underline{E}_{j}\right]_{\underline{c}} .
$$

We use the quantum Serre relations and (54) to write $\underline{E}_{p}^{m_{p i-1}} \underline{E}_{i} \underline{E}_{p}^{m_{p i-1}} \underline{E}_{i} \underline{E}_{p} \underline{E}_{j}$ as a linear combination of greater words modulo $\mathcal{J}\left(s_{p}^{*} \chi\right)$. If $q_{p p} \in \mathbb{G}_{3}$, then

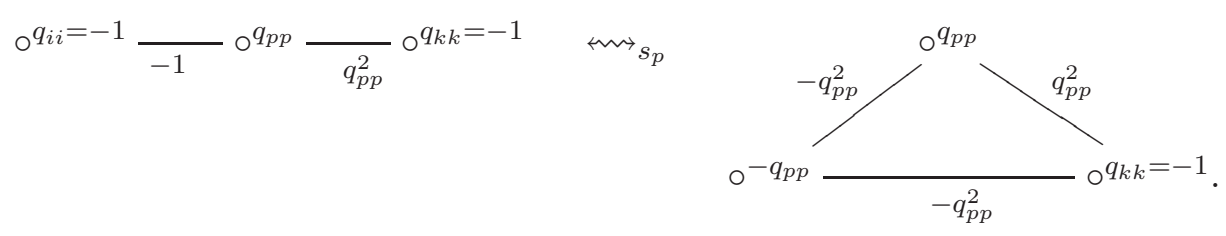

The result follows in a similar way, but using that (45) and (47) are generators of $\mathcal{J}\left(s_{p}^{*} \chi\right)$ in this case.

If $p=i$, by Lemma 3.5, it is equivalent to prove that

$$
T_{p}\left(\mathbf{x}^{\prime}\right) \in \mathcal{J}\left(s_{p}^{*} \chi\right), \quad \mathbf{x}^{\prime}:=\left[E_{k j p}, E_{j p}\right]_{c} .
$$

Note that $\left(\operatorname{ad}_{\underline{c}} \underline{E}_{j}\right)^{2} \underline{E}_{k} \in \mathcal{J}\left(s_{p}^{*} \chi\right)$, because for all the possible diagrams $\underline{q}_{j j}^{-1}=\underline{q}_{j k} \underline{q}_{k j} \neq-1$. By (71), we have

$$
T_{p}\left(\mathbf{x}^{\prime}\right)=a_{1}^{2}\left[\left(\operatorname{ad}_{\underline{c}} \underline{E}_{k}\right) \underline{E}_{j}, \underline{E}_{j}\right]_{\underline{c}} \in \mathcal{J}\left(s_{p}^{*} \chi\right) .
$$

Finally, if $p \neq i, j, k$, then $p$ is not connected with any of these vertices, or $p$ is connected just with $i$ and $m_{p i}=1$, or $p$ is connected just with $k$ and $m_{p k}=1$. The proof is analogous to the corresponding case in previous Lemmata. 
(ii) If $p \neq i, j, k$, then $p$ is not connected with them and the result follows easily. In consequence, we consider the case $p \in\{i, j, k\}$. If $p=k, s_{p}^{*} \chi$ is twist equivalent to $\chi$ and (45) is a generator of $\mathcal{J}\left(s_{p}^{*} \chi\right)$. Therefore (171) implies that

$$
T_{p}\left(\left[E_{i i j k}, E_{i j k}\right]_{c}\right)=a_{2}^{2}\left[\underline{E}_{i i j}, \underline{E}_{i j}\right]_{\underline{c}} \in \mathcal{J}\left(s_{p}^{*} \chi\right) .
$$

If $p=j$, we have that

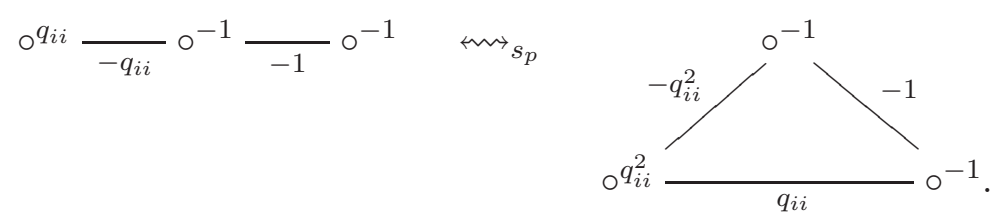

By (71) and fixing the order $p<i<k$, we have that

$$
T_{p}\left(\left[E_{i i j k}, E_{i j k}\right]_{c}\right)=a_{2}^{2}\left[\left[\underline{E}_{p i}, \underline{E}_{p i k}\right]_{\underline{c}}, \underline{E}_{p i k}\right]_{\underline{c}}=\left[\underline{E}_{p} \underline{E}_{i} \underline{E}_{p} \underline{E}_{i} \underline{E}_{k} \underline{E}_{p} \underline{E}_{i} \underline{E}_{k}\right]_{\underline{c}}
$$

We write $\underline{E}_{p} \underline{E}_{i} \underline{E}_{p} \underline{E}_{i} \underline{E}_{k} \underline{E}_{p} \underline{E}_{i} \underline{E}_{k}$ as a linear combination of greater words modulo $\mathcal{J}\left(s_{p}^{*} \chi\right)$ using that (47), (45), $\underline{E}_{p}^{2}, \underline{E}_{k}^{2}$ and $\underline{E}_{i i k}$ are generators of $\mathcal{J}\left(s_{p}^{*} \chi\right)$, so Lemma 3.4 implies that $T_{p}\left(\left[E_{i i j k}, E_{i j k}\right]_{c}\right) \in \mathcal{J}\left(s_{p}^{*} \chi\right)$.

Finally let $p=i$. By Lemma 3.5, it is equivalent to prove that

$$
T_{p}\left(\mathbf{x}^{\prime}\right) \in \mathcal{J}\left(s_{p}^{*} \chi\right), \quad \mathbf{x}^{\prime}:=\left[E_{k j p}, E_{k j p p}\right]_{c} .
$$

Note that $s_{p}^{*} \chi$ is twist equivalent to $\chi$, so $x_{j p}^{2}$ is a generator of $\mathcal{J}\left(s_{p}^{*} \chi\right)$. Applying (71),

$$
T_{p}\left(\mathbf{x}^{\prime}\right)=a_{2}^{2} a_{1}\left[\underline{E}_{k j p}, \underline{E}_{k j}\right]_{c}=a_{2}^{2} a_{1}\left[\underline{E}_{k j}^{2}, \underline{E}_{p}\right]_{c} \in \mathcal{J}\left(s_{p}^{*} \chi\right) .
$$

Therefore $T_{p}\left(\left[E_{i i j k}, E_{i j k}\right]_{c}\right) \in \mathcal{J}\left(s_{p}^{*} \chi\right)$.

Lemma 3.23. (i) Let $i, j, k \in\{1, \ldots, \theta\}$ be such that $q_{i i}=\widetilde{q_{i j}}=-\widetilde{q_{i k}} \in \mathbb{G}_{3}, \widetilde{q_{j k}}=1$, $q_{j j}=-1, q_{k k} \in\left\{-1,{\widetilde{q_{i k}}}^{-1}\right\}$. Then, for all $p$,

$$
T_{p}\left(\left[E_{i},\left[E_{i j}, E_{i k}\right]_{c}\right]_{c}+q_{j k} q_{i k} q_{j i}\left[E_{i i k}, E_{i j}\right]_{c}+q_{i j} E_{i j} E_{i i k}\right) \in \mathcal{J}\left(s_{p}^{*} \chi\right) .
$$

(ii) Let $i, j, k \in\{1, \ldots, \theta\}$ be such that $q_{i i}=q_{k k}=-1, \widetilde{q_{i k}}=1, \widetilde{q_{i j}} \in \mathbb{G}_{3}, q_{j j}=-\widetilde{q_{j k}}=$ $\pm \widetilde{q_{i j}}$. Then, for all $p$,

$$
T_{p}\left(\left[E_{i}, E_{j j k}\right]_{c}-q_{k j}^{-1}\left(1+q_{j j}^{2}\right)\left[E_{i j k}, E_{j}\right]_{c}+\left(1+q_{j j}\right)\left(1+q_{j j}^{2}\right) E_{j} E_{i j k}\right) \in \mathcal{J}\left(s_{p}^{*} \chi\right) .
$$

Proof. If $p \neq i, j, k$, then $p$ is not connected with these three vertices and the proof follows easily for both relations. We consider then $p \in\{i, j, k\}$ for each item.

(i) If $p=k, \underline{E}_{p} \underline{E}_{i} \underline{E}_{p} \underline{E}_{i} \underline{E}_{j} \underline{E}_{i}$ is a linear combination of greater words by (45) and the quantum Serre relations, depending on the value of $q_{k k}$. In this way, there exist $a, b \in \mathbf{k}$ such that

$$
\mathbf{x}^{\prime}:=\left[\underline{E}_{p} \underline{E}_{i} \underline{E}_{p} \underline{E}_{i} \underline{E}_{j} \underline{E}_{i}\right]_{\underline{c}}+a\left[\underline{E}_{p} \underline{E}_{i}^{2} \underline{E}_{p} \underline{E}_{i} \underline{E}_{j}\right]_{\underline{c}}+b\left[\underline{E}_{p} \underline{E}_{i} \underline{E}_{j}\right]_{\underline{c}}\left[\underline{E}_{p} \underline{E}_{i}^{2}\right]_{\underline{c}} \in \mathcal{J}\left(s_{p}^{*} \chi\right) .
$$


On the other hand, by (71) and for the order on the letters $p<i<j$,

$$
\begin{aligned}
T_{p}\left(\left[E_{i},\left[\left(\operatorname{ad}_{c} E_{i}\right) E_{j},\left(\operatorname{ad}_{c} E_{i}\right) E_{p}\right]_{c}\right]_{c}\right) & =a_{1}\left[\left(\operatorname{ad}_{\underline{c}} \underline{E}_{p}\right) \underline{E}_{i},\left[\left(\operatorname{ad}_{\underline{c}} \underline{E}_{p}\right)\left(\operatorname{ad}_{\underline{c}} \underline{E}_{i}\right) \underline{E}_{j}, \underline{E}_{i}\right]_{\underline{c}}\right]_{\underline{c}} \\
& =a_{1}\left[\underline{E}_{p} \underline{E}_{i} \underline{E}_{p} \underline{E}_{i} \underline{E}_{j} \underline{E}_{i}\right]_{\underline{c}}, \\
T_{p}\left(\left[\left(\operatorname{ad}_{c} E_{i}\right)^{2} E_{p},\left(\operatorname{ad}_{c} E_{i}\right) E_{j}\right]_{c}\right) & =a_{1}\left[\left[\left(\operatorname{ad}_{\underline{c}} \underline{E}_{p}\right) \underline{E}_{i}, \underline{E}_{i}\right]_{\underline{c}},\left(\operatorname{ad}_{\underline{c}} \underline{E}_{p}\right)\left(\operatorname{ad}_{\underline{c}} \underline{E}_{i}\right) \underline{E}_{j}\right]_{\underline{c}} \\
& =a_{1}\left[\underline{E}_{p} \underline{E}_{i}^{2} \underline{E}_{p} \underline{E}_{i} \underline{E}_{j}\right]_{\underline{c}} ; \\
T_{p}\left(\left(\operatorname{ad}_{c} E_{i}\right) E_{j}\left(\operatorname{ad}_{c} E_{i}\right)^{2} E_{p}\right) & =a_{1}\left(\operatorname{ad}_{\underline{c}} \underline{E}_{p}\right)\left(\operatorname{ad}_{\underline{c}} \underline{E}_{i}\right) \underline{E}_{j}\left[\left(\operatorname{ad}_{\underline{c}} \underline{E}_{p}\right) \underline{E}_{i}, \underline{E}_{i}\right]_{\underline{c}} \\
& =a_{1}\left[\underline{E}_{p} \underline{E}_{i} \underline{E}_{j}\right]_{\underline{c}}\left[\underline{E}_{p} \underline{E}_{i}^{2}\right]_{\underline{c}} .
\end{aligned}
$$

Calculating explicitly the scalars $a, b$, we notice that $T_{p}(\mathbf{x})=a_{1} \mathbf{x}^{\prime} \in \mathcal{J}\left(s_{p}^{*} \chi\right)$. The case $p=j$ is analogous.

Finally the case $p=i$ follows as the corresponding case in Lemma 3.15.

(ii) The proof is analogous to the previous case.

Lemma 3.24. (i) Let $i, j \in\{1, \ldots, \theta\}$ be such that $m_{i j}, m_{j i}>1$. Then, for all $p$,

$$
T_{p}\left(\left(1-\widetilde{q_{i j}}\right) q_{j j} q_{j i}\left[E_{i},\left[E_{i j}, E_{j}\right]_{c}\right]_{c}-\left(1+q_{j j}\right)\left(1-q_{j j} \widetilde{q_{i j}}\right) E_{i j}^{2}\right) \in \mathcal{J}\left(s_{p}^{*} \chi\right) .
$$

(ii) Let $i, j \in\{1, \ldots, \theta\}$ be such that $q_{j j}=-1, q_{i i} \widetilde{q_{i j}} \notin \mathbb{G}_{6}$ or $m_{j j}=2$, and $q_{i i} \in \mathbb{G}_{4}$, $m_{i j}=4$, or $m_{i j} \in\{4,5\}$. Then, for all $p$,

$$
T_{p}\left(\left[E_{i},\left[E_{i i j}, E_{i j}\right]_{c}\right]_{c}-\frac{1-q_{i i} \widetilde{q_{i j}}-q_{i i}^{2}{\widetilde{q_{i j}}}^{2} q_{j j}}{\left(1-q_{i i} \widetilde{q_{i j}}\right) q_{j i}} E_{i i j}^{2}\right) \in \mathcal{J}\left(s_{p}^{*} \chi\right) .
$$

(iii) Let $i, j \in\{1, \ldots, \theta\}$ be such that $q_{j j}=-1,5 \alpha_{i}+4 \alpha_{j} \in \Delta_{+}^{\chi}$. Let

$$
\begin{aligned}
& v=\widetilde{q_{i j}}, \quad a=(1-v)\left(1-q_{i i}^{4} v^{3}\right)-\left(1-q_{i i} v\right)\left(1+q_{i i}\right) q_{i i} v \\
& b=(1-v)\left(1-q_{i i}^{6} v^{5}\right)-a q_{i i} v \\
& d=\frac{b-\left(1+q_{i i}\right)\left(1-q_{i i} v\right)\left(1+v+q_{i i} v^{2}\right) q_{i i}^{6} v^{4}}{a q_{i i}^{3} q_{i j}^{2} q_{j i}^{3}} .
\end{aligned}
$$

Then, for all $p, T_{p}\left(\left[E_{2 \alpha_{i}+\alpha_{j}}, E_{4 \alpha_{i}+3 \alpha_{j}}\right]_{c}-d E_{3 \alpha_{i}+2 \alpha_{j}}^{2}\right) \in \mathcal{J}\left(s_{p}^{*} \chi\right)$.

Proof. (i) Let $\mathbf{x}$ be the relation we are considering here. We note that if $p \neq i, j$ then $m_{p i}=m_{p j}=0$, so the proof follows easily. Moreover the conditions about $i, j$ are the same but one relation implies the other holds in $\mathcal{U}(\chi)$ too by Lemma 3.4. Therefore it is enough to consider one of cases $p=i$ or $p=j$; consider $p=j$, in order to apply (71). Note that $m_{p i}=2,3$.

If $m_{p i}=3$, then $m_{i p}=2$ and we have that

$$
T_{p}\left(\left[E_{i},\left[E_{i p}, E_{p}\right]_{c}\right]_{c}\right)=\left[\underline{E}_{p p p i}, \underline{E}_{p i}\right]_{\underline{c}} .
$$

By (9) we can write $T_{p}(\mathbf{x})$ as a linear combination of

$$
\left[\underline{E}_{p},\left[\underline{E}_{p p i}, \underline{E}_{p i}\right]_{\underline{c}}\right]_{\underline{c}}, \quad \underline{E}_{p p i}^{2}
$$

note that $\left[\underline{E}_{p},\left[\underline{E}_{p p i}, \underline{E}_{p i}\right]_{\underline{c}}\right]_{\underline{c}}=\left[\underline{E}_{p}^{3} \underline{E}_{i} \underline{E}_{p} \underline{E}_{i}\right]_{\underline{c}}$ if we consider $p<i$. Using the quantum Serre relations or (63), depending on the case (there exist two possible diagrams), $\underline{E}_{p}^{3} \underline{E}_{i} \underline{E}_{p} \underline{E}_{i}$ is 
expressed as a linear combination of greater words modulo $\mathcal{J}\left(s_{p}^{*} \chi\right)$, so $T_{p}(\mathbf{x}) \in \mathcal{J}\left(s_{p}^{*} \chi\right)$ by Lemma 3.4 .

If $m_{p i}=2$, there exist three diagrams such that $m_{i p}=2$, and two such that $m_{i p}=3$. In all the cases,

$$
\begin{aligned}
T_{p}\left(E_{i p}^{2}\right) & =a_{2}^{2} \underline{E}_{p i}^{2}, \\
T_{p}\left(\left[E_{i},\left[E_{i p}, E_{p}\right]_{c}\right]_{c}\right) & =a_{2} a_{1}\left[\underline{E}_{p p i}, \underline{E}_{i}\right]_{\underline{c}}=a_{2} a_{1}\left[\underline{E}_{p},\left[\underline{E}_{p i}, \underline{E}_{i}\right]_{\underline{c}}\right]_{\underline{c}}+a_{2} a_{1} \underline{q}_{p i}\left(\underline{q}_{p p}-\underline{q}_{i i}\right) \underline{E}_{p i}^{2},
\end{aligned}
$$

where we use (9) for the last equality. If $q_{p p}=-\zeta, \widetilde{q_{i p}}=\zeta^{7}, q_{i i}=\zeta^{3}$, for some primitive root $\zeta \in \mathbb{G}_{9}$, then $s_{p}^{*} \chi$ is twist equivalent to $\chi$ and (62) is a generator of $\mathcal{J}\left(s_{p}^{*} \chi\right)$, so $T_{p}(\mathbf{x}) \in \mathcal{J}\left(s_{p}^{*} \chi\right)$ by this relation and Lemma 3.4. For the other braidings $\underline{q}_{i i}=-1$, so $\left[\underline{E}_{p i}, \underline{E}_{i}\right]_{c} \in \mathcal{J}\left(s_{p}^{*} \chi\right)$ and the coefficient of $\underline{E}_{p i}^{2}$ in the expression of $T_{p}(\mathbf{x})$ is zero. Then $T_{p}(\mathbf{x}) \in \overline{\mathcal{J}}\left(s_{p}^{*} \chi\right)$.

(ii) Let $\mathbf{x}$ be the relation we are considering in this item. First we consider $p=j$; if $q_{p p}=-1$, then $m_{p i}=1$, so

$$
s_{p}\left(3 \alpha_{i}+\alpha_{p}\right)=3 \alpha_{i}+2 \alpha_{p}, s_{p}\left(3 \alpha_{i}+2 \alpha_{p}\right)=3 \alpha_{i}+\alpha_{p} \in \Delta_{+}^{s_{p}^{*} \chi}
$$

so $\underline{m}_{i p} \geq 3$. Applying (171) we have that:

$$
\begin{gathered}
T_{p}\left(E_{i i p}^{2}\right)=a_{1}^{2}\left[\underline{E}_{p i}, \underline{E}_{i}\right]_{\underline{c}}^{2}, \\
T_{p}\left(\left[E_{i},\left[E_{i i j}, E_{i j}\right]_{c}\right]_{c}\right)=a_{1}^{2}\left[\underline{E}_{p i},\left[\left[\underline{E}_{p i}, \underline{E}_{i}\right]_{\underline{c}}, \underline{E}_{i}\right]_{\underline{c}}\right]_{\underline{c}} .
\end{gathered}
$$

As $\underline{m}_{i p} \geq 3$, (63) is a generator of $\mathcal{J}\left(s_{p}^{*} \chi\right)$, or $\underline{m}_{i p}=3, \underline{q}_{i i} \notin \mathbb{G}_{4}$, so $\underline{E}_{p} \underline{E}_{i} \underline{E}_{p} \underline{E}_{i}^{3}$ can be written as a linear combination of greater words modulo $\mathcal{J}\left(s_{p}^{*} \chi\right)$, for the order $p<i$, using the corresponding quantum Serre relation and $\underline{E}_{p}^{2}$. In both cases we apply Lemma 3.4 to deduce that $T_{p}(\mathbf{y}) \in \mathcal{J}\left(s_{p}^{*} \chi\right)$.

If $m_{p i}=2$, then $m_{i p}=3$; in this case,

$$
\begin{aligned}
T_{p}\left(E_{i i p}^{2}\right) & =a_{2}^{2}\left[\underline{E}_{p p i}, \underline{E}_{p i}\right]_{\underline{c}}^{2}, \\
T_{p}\left(\left[E_{i},\left[E_{i i p}, E_{i p}\right]_{c}\right]_{c}\right) & =a_{2}^{2}\left[\underline{E}_{p p i}, \underline{E}_{4 \alpha_{p}+3 \alpha_{i}}\right]_{\underline{c}} .
\end{aligned}
$$

We have two possibilities for $s_{p}^{*} \chi$ :

- $\circ^{8} \zeta^{5} \circ^{-1}, \zeta \in \mathbb{G}_{24}$, so (68) is a generator of $\mathcal{J}\left(s_{p}^{*} \chi\right)$,

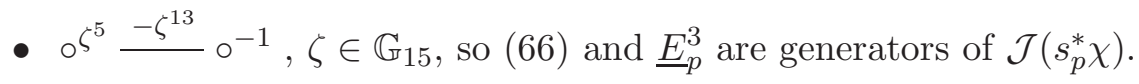

Then $\underline{E}_{p}^{2} \underline{E}_{i} \underline{E}_{p}^{2} \underline{E}_{i} \underline{E}_{p} \underline{E}_{i} \underline{E}_{p} \underline{E}_{i}$ is written as a linear combination of greater words modulo $\mathcal{J}\left(s_{p}^{*} \chi\right)$ in both cases, so by Lemma 3.4 we have that $T_{p}(\mathbf{y}) \in \mathcal{J}\left(s_{p}^{*} \chi\right)$.

Let $p=i$; by Lemma 3.5, it is equivalent to prove that $T_{p}\left(\mathbf{y}^{\prime}\right) \in \mathcal{J}\left(s_{p}^{*} \chi\right)$, where

$$
\mathbf{y}^{\prime}:=\left[\left[E_{j p},\left[E_{j p}, E_{p}\right]_{c}\right]_{c}, E_{p}\right]_{c}-a\left(\left[E_{j p}, E_{p}\right]_{c}\right)^{2},
$$


and $a \in \mathbf{k}^{\times}$is fixed. Note that

$$
\begin{aligned}
& T_{p}\left(\left[\left[\left(\operatorname{ad}_{c} E_{j}\right) E_{p},\left[\left(\operatorname{ad}_{c} E_{j}\right) E_{p}, E_{p}\right]_{c}\right]_{c}, E_{p}\right]_{c}\right) \\
& =a_{m_{p i}}^{2} a_{m_{p i}-1}\left(\left[\left(\operatorname{ad}_{\underline{c}} \underline{E}_{p}\right)^{m_{p i}-1} \underline{E}_{j},\left(\operatorname{ad}_{\underline{c}} \underline{E}_{p}\right)^{m_{p i}-2} \underline{E}_{j}\right]_{\underline{c}} \underline{F}_{p} \underline{L}_{p}^{-1}\right. \\
& \left.\quad-q_{j p}^{2} q_{p p}^{3} \underline{F}_{p} \underline{L}_{p}^{-1}\left[\left(\operatorname{ad}_{\underline{c}} \underline{E}_{p}\right)^{m_{p i}-1} \underline{E}_{j},\left(\operatorname{ad}_{\underline{c}} \underline{E}_{p}\right)^{m_{p i}-2} \underline{E}_{j}\right]_{\underline{c}}\right) \\
& T_{p}\left(\left[E_{j p}, E_{p}\right]_{c}\right)=a_{m_{p i}} a_{m_{p i}-1}\left(\operatorname{ad}_{\underline{c}} \underline{E}_{p}\right)^{m_{p i}-2} \underline{E}_{i}
\end{aligned}
$$

In any case, $T_{p}\left(\mathbf{y}^{\prime}\right) \in \operatorname{ker} \pi_{s_{p}^{*} \chi}$ is a linear combination of

$$
\left[\underline{E}_{p}^{m_{i p}-1} \underline{E}_{i} \underline{E}_{p}^{m_{i p}-3} \underline{E}_{i}\right]_{\underline{c}}, \quad\left[\underline{E}_{p}^{m_{i p}-2} \underline{E}_{i}\right]_{\underline{c}}^{2}
$$

so by Lemma 3.4. $T_{p}\left(\mathbf{y}^{\prime}\right) \in \mathcal{J}\left(s_{p}^{*} \chi\right)$, because (62), (respectively, (63), (66)) is a generator of $\mathcal{J}\left(s_{p}^{*} \chi\right)$ if $m_{p i}=3$, (respectively, $m_{p i}=4, m_{p i}=5$ ).

Finally we take $p \neq i, j$, so $p$ is not connected with $i$ and $j$ (and the proof follows easily by Lemma 3.77), or $p$ is connected only with $i$, and $q_{i i}=\widetilde{q_{i j}}=\widetilde{q_{p i}}-1 \in \mathbb{G}_{4}, q_{p p}=-1$. Consider the order $p<i<j$, so

$$
\begin{aligned}
T_{p}\left(E_{i i j}\right) & =\left[\underline{E}_{p i}, \underline{E}_{p i j}\right]_{\underline{c}}, \\
T_{p}\left(\left[E_{i},\left[E_{i i j}, E_{i j}\right]_{c}\right]_{c}\right) & =\left[\underline{E}_{p i},\left[\left[\underline{E}_{p i}, \underline{E}_{p i j}\right]_{\underline{c}}, \underline{E}_{p i j}\right]_{\underline{c}}\right]_{\underline{c}}=\left[\underline{E}_{p} \underline{E}_{i} \underline{E}_{p} \underline{E}_{i} \underline{E}_{p} \underline{E}_{i} \underline{E}_{j} \underline{E}_{p} \underline{E}_{i} \underline{E}_{j}\right]_{\underline{c}} .
\end{aligned}
$$

By (53), $\underline{E}_{i} \underline{E}_{p} \underline{E}_{i} \underline{E}_{p} \underline{E}_{i} \underline{E}_{j} \underline{E}_{p} \underline{E}_{i}$ can be written as a linear combination of other words modulo $\mathcal{J}\left(s_{p}^{*} \chi\right)$, which are greater than it or they begin with $\underline{E}_{p}$; multiplying on the left by $\underline{E}_{p}$, on the right by $\underline{E}_{j}$, and using that $\underline{E}_{p}^{2} \in \mathcal{J}\left(s_{p}^{*} \chi\right), \underline{E}_{p} \underline{E}_{i} \underline{E}_{p} \underline{E}_{i} \underline{E}_{p} \underline{E}_{i} \underline{E}_{j} \underline{E}_{p} \underline{E}_{i} \underline{E}_{j}$ is a linear combination of greater words modulo $\mathcal{J}\left(s_{p}^{*} \chi\right)$, so $T_{p}(\mathbf{y}) \in \mathcal{J}\left(s_{p}^{*} \chi\right)$ by a similar argument to the previous steps.

(iii) The proof is analogous to the previous items, where we note that in the two possible cases $q_{j j}=-1$, and if $p \neq i, j$, then $p$ is not connected with them.

Lemma 3.25. (i) Let $i, j \in\{1, \ldots, \theta\}$ be such that $4 \alpha_{i}+3 \alpha_{j} \notin \Delta_{+}^{\chi}, q_{j j}=-1$ or $m_{j i}=2$, and also $m_{i j} \geq 3$, or $m_{i j}=2, q_{i i} \in \mathbb{G}_{3}$. Then, $T_{p}\left(\left[E_{3 \alpha_{i}+2 \alpha_{j}}, E_{i j}\right]_{c}\right) \in \mathcal{J}\left(s_{p}^{*} \chi\right)$, for all $p$.

(ii) Let $i, j \in\{1, \ldots, \theta\}$ be such that $4 \alpha_{i}+3 \alpha_{j} \in \Delta_{+}^{\chi}, 5 \alpha_{i}+4 \alpha_{j} \notin \Delta_{+}^{\chi}$. Then, for all $p$, $T_{p}\left(\left[E_{4 \alpha_{i}+3 \alpha_{j}}, E_{i j}\right]_{c}\right) \in \mathcal{J}\left(s_{p}^{*} \chi\right)$.

(iii) Let $i, j \in\{1, \ldots, \theta\}$ be such that $3 \alpha_{i}+2 \alpha_{j} \in \Delta_{+}^{\chi}, 5 \alpha_{i}+3 \alpha_{j} \notin \Delta_{+}^{\chi}$, and $q_{i i}^{3} \widetilde{q_{i j}}, q_{i i}^{4} \widetilde{q_{i j}} \neq 1$. Then, $T_{p}\left(\left[E_{i i j}, E_{3 \alpha_{i}+2 \alpha_{j}}\right]_{c}\right) \in \mathcal{J}\left(s_{p}^{*} \chi\right)$ for all $p$.

(iv) Let $i, j \in\{1, \ldots, \theta\}$ be such that $5 \alpha_{i}+2 \alpha_{j} \in \Delta_{+}^{\chi}, 7 \alpha_{i}+3 \alpha_{j} \notin \Delta_{+}^{\chi}$. Then, for all $p$, $T_{p}\left(\left[\left[E_{i i i j}, E_{i i j}\right], E_{i i j}\right]_{c}\right) \in \mathcal{J}\left(s_{p}^{*} \chi\right)$.

Proof. For these four sets of conditions, if $p \neq i, j$ then $p$ is not connected with $i$ and $j$, so the proof follows easily using Lemma 3.7, or we have a diagram as in Lemma 3.24, (ii), and the proof is analogous to this one. In consequence we will consider $p=i$ and $p=j$ for each one of these cases.

(i) Let $\mathbf{x}=\left[E_{3 \alpha_{i}+2 \alpha_{j}}, E_{i j}\right]_{c}$, and take $p=j$. If $m_{p i}=1$ (that is, $q_{p p}=-1$ or $q_{p p} \widetilde{q_{i p}}=1$ ), we have that

$$
s_{p}\left(3 \alpha_{i}+2 \alpha_{p}\right)=3 \alpha_{i}+\alpha_{p} \in \Delta_{+}^{s_{p}^{*} \chi}, \quad s_{p}\left(4 \alpha_{i}+3 \alpha_{p}\right)=4 \alpha_{i}+\alpha_{p} \notin \Delta_{+}^{s_{p}^{*} \chi} .
$$

Therefore $\underline{m}_{i p}=3$, so $\underline{E}_{i}^{4}$ (respectively, $\left.\left(\operatorname{ad}_{\underline{c}} \underline{E}_{i}\right)^{4} \underline{E}_{p}\right)$ is a generator of $\mathcal{J}\left(s_{p}^{*} \chi\right)$, if $\underline{q}_{i i}$ belongs (respectively, does not belong) to $\mathbb{G}_{4}$. By (71) and the previous relations, depending on 
the case,

$$
T_{p}(\mathbf{x})=a_{1}^{4}\left[\left[\left[\underline{E}_{p i}, \underline{E}_{i}\right]_{\underline{c}}, \underline{E}_{i}\right]_{\underline{c}}, \underline{E}_{i}\right]_{\underline{c}} \in \mathcal{J}\left(s_{p}^{*} \chi\right) .
$$

The remaining case is $m_{p i}=2$, for which there exist two possible diagrams:

$$
\circ^{-\zeta} \stackrel{\zeta^{7}}{-\zeta^{3}}, \zeta \in \mathbb{G}_{9} ; \quad \circ^{-\zeta} \underline{-\zeta^{12}} \circ^{\zeta^{5}}, \zeta \in \mathbb{G}_{15} \text {. }
$$

In both cases $q_{p p} \in \mathbb{G}_{3}$, and also

$$
s_{p}\left(3 \alpha_{i}+2 \alpha_{p}\right)=3 \alpha_{i}+4 \alpha_{p} \in \Delta_{+}^{s_{p}^{*} \chi}, \quad s_{p}\left(4 \alpha_{i}+3 \alpha_{p}\right)=4 \alpha_{i}+5 \alpha_{p} \notin \Delta_{+}^{s_{p}^{*} \chi} .
$$

Then (66) is a generator of $\mathcal{J}\left(s_{p}^{*} \chi\right)$ if $3 \alpha_{i}+5 \alpha_{p} \in \Delta_{+}^{s_{p}^{*} \chi}$, or (67) is a generator of $\mathcal{J}\left(s_{p}^{*} \chi\right)$ in other case, so for both braidings $E_{p}^{2} E_{i} E_{p} E_{i} E_{p} E_{i} E_{p} E_{i}$ is a linear combination of greater words modulo $\mathcal{J}\left(s_{p}^{*} \chi\right)$, and (66) belongs to $\mathcal{J}\left(s_{p}^{*} \chi\right)$. Therefore

$$
T_{p}(\mathbf{x})=a_{2}^{4}\left[\left[\left[\underline{E}_{p p i}, \underline{E}_{p i}\right]_{\underline{c}}, \underline{E}_{p i}\right]_{\underline{c}}, \underline{E}_{p i}\right]_{\underline{c}} \in \mathcal{J}\left(s_{p}^{*} \chi\right) .
$$

Consider now $p=i$, so by Lemma 3.5 it is enough to prove that

$$
T_{p}\left(\mathbf{x}^{\prime}\right) \in \mathcal{J}\left(s_{p}^{*} \chi\right), \quad \mathbf{x}^{\prime}:=\left[E_{j p},\left[E_{j p},\left[E_{j p}, E_{p}\right]_{c}\right]_{c}\right]_{c} .
$$

If $m_{p j}=2$, then $s_{p}\left(3 \alpha_{p}+2 \alpha_{j}\right)=\alpha_{p}+2 \alpha_{j} \in \Delta_{+}^{s_{p}^{*} \chi}, s_{p}\left(4 \alpha_{p}+3 \alpha_{j}\right)=2 \alpha_{p}+3 \alpha_{j} \notin \Delta_{+}^{s_{p}^{*} \chi}$, so $\underline{m}_{j p}=2$, and (45) is a generator of $\mathcal{J}\left(s_{p}^{*} \chi\right)$; then

$$
T_{p}\left(\mathbf{x}^{\prime}\right)=a_{2}^{3} a_{1}\left[\underline{E}_{p j},\left[\underline{E}_{p j}, \underline{E}_{j}\right]_{\underline{c}}\right]_{\underline{c}} \in \mathcal{J}\left(s_{p}^{*} \chi\right) .
$$

If $m_{p j}=3$, then $s_{p}\left(3 \alpha_{p}+2 \alpha_{j}\right)=3 \alpha_{p}+2 \alpha_{j} \in \Delta_{+}^{s_{p}^{*} \chi}, s_{p}\left(4 \alpha_{p}+3 \alpha_{j}\right)=5 \alpha_{p}+3 \alpha_{j} \notin \Delta_{+}^{s_{p}^{*} \chi}$, so (67) is a generator of $\mathcal{J}\left(s_{p}^{*} \chi\right)$. By (71),

$$
T_{p}\left(\mathbf{x}^{\prime}\right)=a_{3}^{3} a_{2}\left[\underline{E}_{p p j},\left[\underline{E}_{p p j}, \underline{E}_{p j}\right]_{\underline{c}}\right]_{\underline{c}} \in \mathcal{J}\left(s_{p}^{*} \chi\right) .
$$

If $m_{p j}=4$, then $s_{p}\left(3 \alpha_{p}+2 \alpha_{j}\right)=5 \alpha_{p}+2 \alpha_{j} \in \Delta_{+}^{s_{p}^{*} \chi}$; moreover, we note that $7 \alpha_{p}+3 \alpha_{j} \notin$ $\Delta_{+}^{s_{p}^{*} \chi}$ in both cases, and (65) is a generator of $\mathcal{J}\left(s_{p}^{*} \chi\right)$. By this relation and the quantum Serre relations, $\underline{E}_{p}^{3} \underline{E}_{j} \underline{E}_{p}^{3} \underline{E}_{j} \underline{E}_{p}^{2} \underline{E}_{j}$ is written as a linear combination of greater words modulo $\mathcal{J}\left(s_{p}^{*} \chi\right)$, so

$$
T_{p}\left(\mathbf{x}^{\prime}\right)=a_{4}^{3} a_{3}\left[\underline{E}_{p p p j},\left[\underline{E}_{p p p j}, \underline{E}_{p p j}\right]_{\underline{c}}\right]_{\underline{c}} \in \mathcal{J}\left(s_{p}^{*} \chi\right) .
$$

(ii) The proof is similar to (i), but more simple: for $p=j$ we have just one possibility, $m_{p i}=1$.

(iii) Let $\mathbf{x}=\left[E_{i i j}, E_{3 \alpha_{i}+2 \alpha_{j}}\right]_{c}$. Consider $p=j$; we consider $i$ a non-Cartan vertex, because in other case $\underline{E}_{i} \underline{E}_{p} \underline{E}_{i}^{2}$ or $\underline{E}_{i}^{2} \underline{E}_{p} \underline{E}_{i}^{2}$ can be written as a linear combination of other words using the corresponding quantum Serre relation, and finally $\underline{E}_{i}^{2} \underline{E}_{p} \underline{E}_{i}^{2} \underline{E}_{p} \underline{E}_{i}$ is a linear combination of greater words modulo $\mathcal{J}\left(s_{p}^{*} \chi\right)$, so the relation is redundant in this case. In consequence we consider $m_{p i}=1$, and for this case $\underline{E}_{p} \underline{E}_{i}^{2} \underline{E}_{p} \underline{E}_{i}^{3}$ is a linear combination of greater words modulo $\mathcal{J}\left(s_{p}^{*} \chi\right)$, so

$$
T_{p}(\mathbf{x})=\left[\left[\underline{E}_{p i}, \underline{E}_{i}\right]_{\underline{c}},\left[\left[\underline{E}_{p i}, \underline{E}_{i}\right]_{\underline{c}}, \underline{E}_{i}\right]_{\underline{c}}\right]_{\underline{c}} \in \mathcal{J}\left(s_{p}^{*} \chi\right)
$$

Let $p=i$; as above, it is enough to prove that

$$
T_{p}\left(\mathbf{x}^{\prime}\right) \in \mathcal{J}\left(s_{p}^{*} \chi\right), \quad \mathbf{x}^{\prime}:=\left[\left[E_{j p},\left[E_{j p}, E_{p}\right]_{c}\right]_{c},\left[E_{j p}, E_{p}\right]_{c}\right]_{c} .
$$


If $m_{p j}=2$, then $s_{p}\left(3 \alpha_{p}+2 \alpha_{j}\right)=\alpha_{p}+2 \alpha_{j} \in \Delta_{+}^{s_{p}^{*} \chi}, s_{p}\left(5 \alpha_{p}+3 \alpha_{j}\right)=\alpha_{p}+3 \alpha_{j} \notin \Delta_{+}^{s_{p}^{*} \chi}$, so $\left(\operatorname{ad}_{\underline{c}} \underline{E}_{j}\right)^{3} \underline{E}_{p}$ (or $\left.\underline{E}_{i}^{3}\right)$ is a generator of $\mathcal{J}\left(s_{p}^{*} \chi\right)$; therefore

$$
T_{p}\left(\mathbf{x}^{\prime}\right)=a_{2}^{3} a_{1}^{2}\left[\left[\underline{E}_{p j}, \underline{E}_{j}\right]_{\underline{c}}, \underline{E}_{j}\right]_{\underline{c}} \in \mathcal{J}\left(s_{p}^{*} \chi\right) .
$$

If $m_{p j}=3$, then $s_{p}\left(3 \alpha_{p}+2 \alpha_{j}\right)=3 \alpha_{p}+2 \alpha_{j} \in \Delta_{+}^{s_{p}^{*} \chi}, s_{p}\left(5 \alpha_{p}+3 \alpha_{j}\right)=4 \alpha_{p}+3 \alpha_{j} \notin \Delta_{+}^{s_{p}^{*} \chi}$, so (66) is a generator of $\mathcal{J}\left(s_{p}^{*} \chi\right)$. Therefore

$$
T_{p}\left(\mathbf{x}^{\prime}\right)=a_{3}^{3} a_{2}^{2}\left[\left[\underline{E}_{p p j}, \underline{E}_{p j}\right]_{\underline{c}}, \underline{E}_{p j}\right]_{\underline{c}} \in \mathcal{J}\left(s_{p}^{*} \chi\right) .
$$

If $m_{p j}=4$, then $s_{p}\left(3 \alpha_{p}+2 \alpha_{j}\right)=5 \alpha_{p}+2 \alpha_{j} \in \Delta_{+}^{s_{p}^{*} \chi}, s_{p}\left(5 \alpha_{p}+3 \alpha_{j}\right)=7 \alpha_{p}+3 \alpha_{j} \notin \Delta_{+}^{s_{p}^{*} \chi}$, so (65) is a generator of $\mathcal{J}\left(s_{p}^{*} \chi\right)$. In consequence,

$$
T_{p}\left(\mathbf{x}^{\prime}\right)=a_{4}^{3} a_{3}^{2}\left[\left[\underline{E}_{p p p j}, \underline{E}_{p p j}\right]_{\underline{c}}, \underline{E}_{p p j}\right]_{\underline{c}} \in \mathcal{J}\left(s_{p}^{*} \chi\right) .
$$

(iv) The proof is analogous to the previous one.

Now we are ready to prove that the Lusztig isomorphisms descend to the family of algebras $U(\chi)$, so we will look at the root system of this family of algebras. As we consider finite root systems, they are univocally determined as sets of real roots, and using this result we will obtain the desired Theorem of presentation by generators and relations of Nichols algebras.

Proposition 3.26. The morphisms (38) induce algebra isomorphisms

$$
T_{p}, T_{p}^{-}: U(\chi) \rightarrow U\left(s_{p}^{*} \chi\right),
$$

such that $T_{p} T_{p}^{-}=T_{p}^{-} T_{p}=\operatorname{id}_{U(\chi)}$.

Proof. By the definition of the ideals $\mathcal{J}(\chi)$ and the previous Lemmata, $T_{p}(\mathcal{J}(\chi)) \subset$ $\mathcal{J}\left(s_{p}^{*} \chi\right)$, so there exists an algebra morphism $T_{p}: U(\chi) \rightarrow U\left(s_{p}^{*} \chi\right)$. By $\phi_{4}^{2}=$ id and the definition of the ideal, $\phi_{4}(\mathcal{J}(\chi))=\mathcal{J}(\chi)$, and also $\varphi_{\lambda}(\mathcal{J}(\chi))=\mathcal{J}(\chi)$ for any $\lambda \in\left(\mathbf{k}^{\times}\right)^{\theta}$, because the ideal is $\mathbb{Z}^{\theta}$-homogeneous. By (39) we have that $T_{p}^{-}(\mathcal{J}(\chi)) \subset \mathcal{J}\left(s_{p}^{*} \chi\right)$, so there exists also an algebra morphism $T_{p}^{-}: U(\chi) \rightarrow U\left(s_{p}^{*} \chi\right)$, induced by the corresponding morphism.

These algebras are generated by $E_{i}, F_{i}, L_{i}, K_{i}$, and the identities $T_{p} T_{p}^{-}=T_{p}^{-} T_{p}=$ id hold for each one of these elements, so these identities hold for all the elements of these algebras, and these morphisms are isomorphisms.

This result lets us to prove the main result of this Section. The proof is similar to the one for [A1, Thm.5.25].

\section{Proof of Theorem 3.1.}

Set $\underline{\Delta}_{+}^{\chi}:=\Delta^{+}\left(U^{+}(\chi)\right) \backslash\left\{N_{\alpha} \alpha: \alpha \in \Delta_{+}^{\chi}\right\}$. By the triangular decomposition, Lemma 2.8, Theorem 2.9 and Proposition 3.26, we have that

$$
\mathcal{H}_{U^{+}(\chi)}=\mathcal{H}_{U_{+p}^{+}(\chi)} \mathfrak{q}_{h\left(E_{p}\right)}=s_{p}\left(\mathcal{H}_{U_{+p}^{+}\left(s_{p}^{*} \chi\right)}\right) \mathfrak{q}_{h\left(E_{p}\right)},
$$

for all $p \in\{1, \ldots, \theta\}$, because $\operatorname{deg}\left(T_{p}(X)\right)=s_{p}(\operatorname{deg} X)$ for each homogeneous element $X \in \mathcal{U}(\chi)$. Recall that $h\left(E_{p}\right) \in\left\{\right.$ ord $\left.q_{p p}, \infty\right\}$, so

$$
\Delta^{+}\left(U^{+}(\chi)\right)=s_{p}\left(\Delta^{+}\left(U^{+}\left(s_{p}^{*} \chi\right)\right) \backslash\left\{\alpha_{p}, N_{p} \alpha_{p}\right\}\right) \cup S_{p},
$$

where $S_{p}=\left\{\alpha_{p}\right\}$, or $S_{p}=\left\{\alpha_{p}, N_{p} \alpha_{p}\right\}$, so $\underline{\Delta}_{+}^{\chi}=s_{p}\left(\underline{\Delta}_{+}^{s_{p}^{*} \chi} \backslash\left\{\alpha_{p}\right\}\right) \cup\left\{\alpha_{p}\right\}$. 
In this way, if we consider the sets $\underline{\Delta}_{+}^{\chi}$, for each $\chi$ in a Weyl equivalence class of a fixed braiding with finite root system, then $R=\left\{\underline{\Delta}_{+}^{\chi}\right\}$ is a root system for our Weyl groupoid, according with the Definition 1.17. As we have a finite root system, it follows that $\underline{\Delta}_{+}^{\chi}=\Delta_{+}^{\chi}$, for all $\chi$, because by Proposition 1.21 all the roots are real. In this way, $\Delta^{+}\left(U^{+}(\chi)\right)$ is obtained from $\Delta_{+}^{\chi}$ adding $N_{\alpha} \alpha$, for some $\alpha \in \Delta_{+}^{\chi}$. Fix an order on the letters $x_{i}$ and consider the corresponding PBW basis. We have a projection $\pi_{\chi}: U(\chi) \rightarrow \mathfrak{u}(\chi)$ of graded braided Hopf algebras, so the corresponding $x_{\alpha}$ of the PBW basis of $\mathfrak{u}(\chi)$ are generators of the PBW basis of $U(\chi)$, by the definition of Kharchenko's PBW basis. On the other hand, each simple root of a non-Cartan vertex satisfies $E_{i}^{N_{i}}=0$ in $U(\chi)$, so $N_{i} \alpha_{i} \notin \Delta^{+}\left(U^{+}(\chi)\right)$. Therefore (72) implies that

$$
N_{\alpha} \alpha \notin \Delta^{+}\left(U^{+}(\chi)\right), \quad \text { for all } \alpha \in \Delta_{+}^{\chi} \backslash \mathcal{O}(\chi),
$$

because $\alpha$ is of the way $\alpha=w\left(\alpha_{i}\right)$ for some $w \in \mathcal{W}$ and $i \in\{1, \ldots, \theta\}, i$ a non-Cartan vertex in the corresponding $\chi^{\prime}$. Analogously, for each Cartan vertex $i, N_{i} \alpha_{i} \in \Delta^{+}\left(U^{+}(\chi)\right)$, because $E_{i}^{N_{i}} \neq 0$ in $U(\chi)$, so

$$
N_{\alpha} \alpha \in \Delta^{+}\left(U^{+}(\chi)\right), \quad \text { for all } \alpha \in \mathcal{O}(\chi) .
$$

Therefore $\Delta^{+}\left(U^{+}(\chi)\right)=\Delta_{+}^{\chi} \cup\left\{N_{\alpha} \alpha: \alpha \in \mathcal{O}(\chi)\right\}$.

Suppose that the degree $N_{\alpha} \alpha$ in $\Delta^{+}\left(U^{+}(\chi)\right)$ corresponds to a Lyndon word of this degree: we can assume that it is of minimal length, and we denote it by $u$; set $(v, w)=$ $\operatorname{Sh}(u)$. In this way, $\operatorname{deg} v=\beta$, $\operatorname{deg} w=\gamma$, for some $\beta, \gamma \in \Delta_{+}^{\chi}$, and $\beta+\gamma=N_{\alpha} \alpha$. As all the roots are real, we deduce that if $\beta<\gamma$, then $\beta<\alpha<\gamma$, by a similar argument to the convexity properties in A2]. We can consider then the case $\beta=\alpha_{i}$, because if $\beta=s_{i_{1}} \cdots s_{i_{k}}\left(\alpha_{i_{k+1}}\right)$, where $w=s_{i_{1}} \cdots s_{i_{k}}$ is the beginning of the expression of the element of maximal length, we apply $w^{-1}$ to obtain that $\alpha_{i_{k+1}}+\gamma^{\prime}=N_{\alpha} \alpha^{\prime}$ for some $\alpha^{\prime}, \gamma^{\prime} \in \Delta_{+}^{\chi}$. Note also that $N_{\alpha}>2$, because if we suppose $N_{\alpha}=2$, then $\alpha$ is applied in a simple root $\alpha_{i}$ corresponding a Cartan vertex by some element of the Weyl groupoid, and as $N_{\alpha}$ is invariant by the action of the Weyl groupoid, it should be $q_{i i}=-1$, but it corresponds to an isolated vertex or a non-Cartan vertex, which is a contradiction. Set

$$
\alpha=\sum_{j=1}^{\theta} n_{j} \alpha_{j}, \quad \gamma=\sum_{j=1}^{\theta} m_{j} \alpha_{j}, \quad \text { for some } n_{j}, m_{j} \in \mathbb{N}_{0} .
$$

Note that $m_{i}=N_{\alpha} n_{i}-1 \geq 2$, and for $j \neq i, m_{j}=N_{\alpha} n_{j} \geq 3$, so $\operatorname{supp} \gamma=\operatorname{supp} \alpha$. By simplicity assume that $\operatorname{supp} \alpha=\{1, \ldots, \theta\}$; note that the vertices of $\operatorname{supp} \beta$ corresponds to a connected subdiagram, for any positive root $\beta$.

By these considerations we reduce the problem to an analysis case by case of the positive root systems of connected diagrams, and we do it with the help of the program SARNA [GHV]. We look for the possible $\gamma$ such that all the coordinates, except at most one, are divisible by an integer $\geq 3$, and the remaining coordinate is $\geq 2$, so we just have a few 3-uples in rank two or three. Analyzing each of these 3-uples

$$
(\alpha, \gamma, i) \in \Delta_{+}^{\chi} \times \Delta_{+}^{\chi} \times\{1, \ldots, \theta\} \text { such that there exists } N \in \mathbb{N}: \alpha_{i}+\gamma=N \alpha,
$$

we note that $N \neq N_{\alpha}$ for all of them. Therefore, there are no Lyndon words of degree $N_{\alpha} \alpha$, so the generators of degree $N_{\alpha} \alpha$ are $x_{\alpha}^{N_{\alpha}}$, and then the elements

$$
x_{\beta_{1}}^{n_{1}} \cdots x_{\beta_{k}}^{n_{k}}, \quad \beta_{i} \in \Delta_{+}^{\chi}, \quad \begin{cases}0 \leq n_{j}<N_{j}, & \text { if } \beta_{j} \notin \mathcal{O}(\chi) \\ 0 \leq n_{j}<\infty, & \text { si } \beta_{j} \in \mathcal{O}(\chi)\end{cases}
$$

are a PBW basis of $U(\chi)$. As $x_{\alpha}^{N_{\alpha}}=0$ in $\mathfrak{u}^{+}(\chi), \pi_{\chi}$ induces a surjective morphism

$$
\pi_{\chi}^{\prime}: U(\chi) /\left\langle x_{\alpha}^{N_{\alpha}}: \alpha \in \mathcal{O}(\chi)\right\rangle \longrightarrow \mathfrak{u}^{+}(\chi),
$$


which applies the set

$$
\left\{x_{\beta_{1}}^{n_{1}} \cdots x_{\beta_{k}}^{n_{k}}, \quad \beta_{i} \in \Delta_{+}^{\chi}, 0 \leq n_{j}<N_{j}\right\},
$$

generating linearly the quotient, to the corresponding PBW basis of $\mathfrak{u}^{+}(\chi)$. Therefore $\pi_{\chi}^{\prime}$ is an isomorphism.

\section{Generation in Degree one}

Now we answer positively the Conjecture1, formulated by Andruskiewitsch and Schneider, but restricting to the case in which $G(H)$ is abelian. The technique of the proof is the same that these authors use in AS4, extended in some works to other families. In particular, the first Lemmata of this Section correspond to relations generating the ideal for standard braidings as in AGI, but the proof is made in a general context.

In what follows $\Gamma$ denotes a finite abelian group, and $S=\bigoplus_{n \geq 0} S(n)$ is a graded braided Hopf algebra in ${ }_{G}^{G} \mathcal{Y D}$ such that $S(0)=\mathbf{k} 1$, generated as an algebra by $V:=S(1)$. Fix a basis $\left\{x_{1}, \ldots, x_{\theta}\right\}$ of $V$, so $V$ has a braiding of diagonal type: we can assume that $x_{i} \in S(1)_{g_{i}}^{\chi_{i}}$ for some $g_{i} \in \Gamma$ and $\chi_{i} \in \widehat{\Gamma}$. Set then $q_{i j}:=\chi_{j}\left(g_{i}\right) \in \mathbf{k}^{\times}$.

We will prove that if $S$ is finite dimensional, then $S$ is the Nichols algebra $\mathcal{B}(V)$ associated to $V$. We will obtain then the main Theorem of this Section, answering this Conjecture.

We begin by extending [AS4, Lemma 5.4] for a general quantum Serre relation, proving that they hold in $S$, or $S$ is infinite-dimensional.

Proposition 4.1. Let $S$ be as above. If there exist $i, j \in\{1, \ldots, \theta\}$ such that $q_{i i}^{m_{i j}+1} \neq 1$, and also $\operatorname{ad}_{c}\left(x_{i}\right)^{1+m_{i j}}\left(x_{j}\right) \neq 0$, then $S$ is infinite-dimensional.

Proof. By definition of $m_{i j}$, we have that $q_{i i}^{m_{i j}} \widetilde{q_{i j}}=1$. We begin the proof as in AS4, Lemma 5.4]. To simplify the notation, call $m=m_{i j}, q=q_{i i}, y_{1}:=x_{i}, y_{2}:=x_{j}, y_{3}:=$ $\operatorname{ad}_{c}\left(x_{i}\right)^{1+m}\left(x_{j}\right)$. Set also

$$
\begin{array}{lll}
h_{1}=g_{i}, & h_{2}=g_{j}, & h_{3}=g_{i}^{m+1} g_{j}, \\
\eta_{1}=\chi_{i}, & \eta_{2}=\chi_{j}, & \eta_{3}=\chi_{i}^{m+1} \chi_{j},
\end{array}
$$

so $y_{k} \in S_{h_{k}}^{\eta_{k}}, 1 \leq k \leq 3$. If $W=\mathbf{k} y_{1}+\mathbf{k} y_{2}+\mathbf{k} y_{3}$, then $W \subset \mathcal{P}(S)$ (because $y_{3}$ is also primitive), so there exists a monomorphism $\mathcal{B}(W) \hookrightarrow S$. We compute the corresponding braiding matrix $\left(Q_{k l}=\eta_{l}\left(h_{k}\right)\right)_{1 \leq k, l \leq 3}$, and consider the corresponding generalized Dynkin diagram:

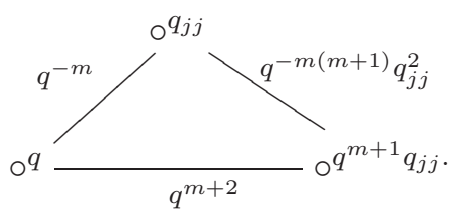

We will consider the different possible cases and prove that no one of them are in [H3], so $\mathcal{B}(W)$, and in consequence $S$, is infinite-dimensional. Suppose that the diagram (74) is in Heckenberger's list:

Case I: $Q_{k l} Q_{l k} \neq 1$ for all $1 \leq k<l \leq 3$. By [H3, Lemma 9], $1=\prod_{k<l} Q_{k l} Q_{l k}=$ $q^{2-m(m+1)} q_{j j}^{2}$, and at least one of the vertices is labeled with -1 . Note that $q \neq-1$, because in such case $m=1$ (and we assume $q^{m+1} \neq 1$ ). Also $q_{j j} \neq q^{m+1} q_{j j}$ by hypothesis, so there is only one vertex labeled with -1 .

- If $q_{j j}=-1$, then $1=\left(q^{m+1} q_{j j}\right)\left(q^{-m(m+1)} q_{j j}^{2}\right)=-q^{1-m^{2}}$ and $m=1$ by the same Heckenberger's Lemma, which is a contradiction. 
- If $q^{m+1} q_{j j}=-1$, then $1=q q^{m+2}=q^{m+3}$ by the same result, and also

$$
1=q_{j j}\left(q^{-m(m+1)} q_{j j}^{2}\right)=q_{j j}^{3} q^{-m(m+3)+2 m}=q_{j j}^{3} q^{2 m},
$$

so we deduce that

$$
-1=(-1)^{3}=q_{j j}^{3} q^{3 m+3}=\left(q_{j j}^{3} q^{2 m}\right) q^{m+3}=q^{m+3},
$$

which is also a contradiction. Therefore (74) does not belong to Heckenberger list for this case.

Case II: $Q_{12} Q_{21}=q^{-m}=1$. In this case $m=0$, so (74) becomes:

$$
{ }^{q} \underset{q^{2}}{{ }^{q q} q_{j j}} \frac{}{q_{j j}^{2}} \circ^{q_{j j}} .
$$

If $q_{j j}=-1$ we obtain the connected subdiagram $\circ^{q} \frac{{ }^{2}}{q^{-q}}$, which has no vertices labeled with -1 , and these labels are different. Such diagram is not of finite Cartan type and moreover it does not correspond to any diagram without -1 in the vertices in rows $5,9,11,12,15$ of [H3, Table 1$]$, so $\mathcal{B}(W)$ is infinite-dimensional.

If $q_{j j} \neq-1$ and $q=-1$, we have an analogous situation, so $q \neq-1$ and (75) is a connected diagram of three vertices. If $q q_{j j} \neq-1$, then [H3, Lemma 9] implies that one of the following situations holds:

- the diagram is of finite Cartan type, so it contains a subdiagram of Cartan type $A_{2}$. Then $1=q q^{2}=\left(q q_{j j}\right) q^{2}$, or $1=q_{j j} q_{j j}^{2}=\left(q q_{j j}\right) q_{j j}^{2}$, so $q=1$ or $q_{j j}=1$;

- $q^{3}=1, q_{j j}, q_{j j} q \in \mathbb{G}_{6} \cup \mathbb{G}_{9}$, and $q_{j j} q_{j j}^{2}=1$ or $q_{j j}^{3}=1, q, q_{j j} q \in \mathbb{G}_{6} \cup \mathbb{G}_{9}, q q^{2}=1$.

No one of these situations hold, so $q q_{j j}=-1$. If this diagram is in [H3, Table 2], it follows that $Q_{i i} Q_{i 2} Q_{2 i}=1$ for some $i \in\{1,3\}$ in all the possible cases. We can assume then $i=1$, $q^{3}=1$. By [H3, Lemma 9], one of the following situations holds:

- $q_{j j}^{3}=1$, but also $q_{j j}^{3}=-q^{-3}=-1$,

- $q_{j j}^{4}=1$,

- $q_{j j}=-q$.

No one of these situations are possible, so we obtain a contradiction in this case too.

Case III: $Q_{13} Q_{31}=q^{m+2}=1$. We obtain the diagram:

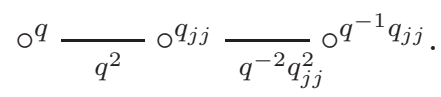

Such diagram is the corresponding to (75), but changing $q_{j j}$ by $q_{j j} q^{-1}$, so it does not belong to [H3, Table 2]. Then $q^{m+2} \neq 1$.

Case $\boldsymbol{I} \boldsymbol{V}: Q_{23} Q_{32}=1$. This means $q_{j j}^{2}=q^{m(m+1)}$, so we have the diagram:

$$
\circ^{q_{j j}} \frac{{ }^{-m}}{q^{q}} \frac{{ }_{q^{m+2}} q^{m+1} q_{j j}}{} .
$$

This diagram is connected by the previous cases. As $m \neq 0, q^{m+1} \neq 1$, it follows that $q \neq-1$. Consider the different possible values of the labels of the vertices:

$\mathbf{q}_{\mathbf{j} \mathbf{j}}=\mathbf{q}^{\mathbf{m + 1}} \mathbf{q}_{\mathbf{j j}}=-\mathbf{1}$ : that is, $q^{m+1}=1$ and we have the diagram:

$$
\circ^{-1} \frac{{ }^{q}}{q}{ }^{q} \circ^{-1},
$$

which is not in Heckenberger's list. 
$\mathbf{q}_{\mathbf{j j}}=-\mathbf{1}, \mathbf{q}^{\mathbf{m}+\mathbf{1}} \mathbf{q}_{\mathbf{j} \mathbf{j}} \neq-\mathbf{1}$ : By [H3, Table 2], it should be $1=Q_{22} Q_{23} Q_{32}=q^{m+3}$, and we should have the diagram

$$
\circ^{-q^{-2}} \frac{}{q^{-1}} \circ^{q} \frac{{ }^{-1}}{q^{3}} \text {. }
$$

Moreover, $1=q_{j j}^{2}=q^{m(m+1)}=q^{2 m}=q^{-6}$. Note that $q^{3} \neq 1$ because $q^{m} \neq 1$, so $q \in \mathbb{G}_{6}$. But this diagram is not in Heckenberger's list.

$\mathbf{q}_{\mathbf{j} \mathbf{j}} \neq-\mathbf{1}, \mathbf{q}^{\mathbf{m}+\mathbf{1}} \mathbf{q}_{\mathbf{j j}}=-\mathbf{1}$ : as above, $1=Q_{22} Q_{21} Q_{12}=q^{1-m}$. By definition it should be $m=1$, with the same diagram of the previous case and $q \in \mathbb{G}_{6}$, so we obtain the same contradiction.

$\mathbf{q}_{\mathbf{j} \mathbf{j}}, \mathbf{q}^{\mathbf{m}+\mathbf{1}} \mathbf{q}_{\mathbf{j} \mathbf{j}} \neq-\mathbf{1}$ : By [H3, Lemma 9], one of the following situations holds:

- the diagram is of Cartan type. Then, $q=q_{j j}$ and $m=1$, or $q=q^{m+1} q_{j j}=q^{-m-2}$. In both cases we obtain the same diagram,

$$
\circ^{q} \frac{{ }^{q}}{q^{-1}} \circ_{q^{3}}^{q^{3}} .
$$

We discard easily the cases $A_{3}, C_{3}$, because $q, q^{2} \neq q^{3}$. If it is of type $B_{3}, q=$ $\left(q^{3}\right)^{2}=q^{-3}$, which is a contradiction.

- $q_{j j} \in \mathbb{G}_{3}, q \in \mathbb{G}_{6} \cup \mathbb{G}_{9}$ and $1=q^{1-m}=q_{j j} q^{2 m+3}$. Then $m=1$ and $q^{5}=q_{j j}^{-1}$, so $q^{15}=1$, but we obtain then a contradiction with the fact that $q \in \mathbb{G}_{6} \cup \mathbb{G}_{9}$ is primitive.

- $q^{m+1} q_{j j} \in \mathbb{G}_{3}, q \in \mathbb{G}_{6} \cup \mathbb{G}_{9}$ y $1=q_{j j} q^{-m}=q^{m+3}$. Again $q^{15}=1$, and we obtain the same contradiction.

In consequence, (74) is not in Heckenberger's list, and $S$ is infinite-dimensional.

Now we continue with another Lemmata from [AGI], just adapted to this general context.

Lemma 4.2. Let $j, k, l \in\{1, \ldots, \theta\}$ be such that $q_{k k}=-1, \widetilde{q_{k j}}=\widetilde{q_{k l}}-1 \neq 1, \widetilde{q_{j l}}=1$. If $\left[x_{j k l}, x_{k}\right]_{c} \neq 0$ is a primitive element of $S$, then $S$ is infinite-dimensional.

Proof. Set $u:=\left[x_{j k l}, x_{k}\right]_{c}, g_{u}:=g_{j} g_{k}^{2} g_{l} \in \Gamma, \chi_{u}:=\chi_{j} \chi_{k}^{2} \chi_{l} \in \widehat{\Gamma}, q:=\widetilde{q_{l k}}$; we work then as in the previous Lemma.

We compute the braiding corresponding to the primitive elements $y_{1}=x_{j}, y_{2}=x_{k}$, $y_{3}=x_{l}$ and $y_{4}=u$, with the corresponding elements $h_{i} \in \Gamma, \eta_{i} \in \widehat{\Gamma}$; we will prove that such braiding has an associated Nichols algebra of infinite dimension, and so $S$ has infinite dimension. The corresponding generalized Dynkin diagram to $\left(Q_{r s}=\eta_{s}\left(h_{r}\right)\right)_{1 \leq r, s \leq 4}$ is:

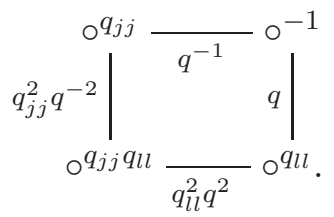

Suppose that such diagram is in Heckenberger's list. If $q=-1$, then (177) contains (75) as a subdiagram, so it does not appear in the list. Therefore $q \neq-1$. As each diagram in [H3, Table 3] does not contain a 4-cycle, it follows that $q_{j j}^{2} q^{-2}=1$, or $q_{l l}^{2} q^{2}=1$. As the conditions are symmetric, it is enough to consider the case $q_{j j}= \pm q$.

If we also have $q_{l l}= \pm q^{-1}$, and as $Q_{44}=q_{j j} q_{l l} \neq 1$, the diagram contains the following

$$
{ }^{q} \frac{}{q^{-1}} \circ^{-1} \frac{}{q} \circ^{-q^{-1}},
$$


which is a contradiction with [H3, Lemma 9]. In consequence we have:

$$
\circ^{ \pm q} \frac{{ }_{q^{-1}}}{-1} \frac{}{q} \circ^{q_{l l}} \frac{q_{l l}^{2} q^{2}}{{ }^{q_{j j}} q_{l l}}
$$

Suppose that $q_{j j}=-q$. As $Q_{11} Q_{12} Q_{21} \neq 1$, we deduce from [H3, Table 3] that $m_{12}=2$; that is,

$$
0=\left(1-Q_{11}^{3}\right)\left(Q_{11}^{2} Q_{12} Q_{21}-1\right)=\left(1+q^{3}\right)(q-1),
$$

which gives conditions about $q$, but each diagram in [H3, Lemma 9] does not satisfy this condition.

Therefore $q_{j j}=q$. We look at [H3, Table 3] but a diagram in such list does not satisfy $Q_{22}=-1, Q_{11}=Q_{44} Q_{33}^{-1}=q \neq \pm 1$, so (77) is not in the list. In consequence, $S$ has infinite dimension.

Lemma 4.3. (i) Let $i, j \in\{1, \ldots, \theta\}$ be such that $q_{j j}=-1, q_{i i} \widetilde{q_{i j}} \in \mathbb{G}_{6}$, and also $q_{i i} \in \mathbb{G}_{3}$ or $m_{i j} \geq 3$. If $\left[x_{i i j}, x_{i j}\right]_{c} \in \mathcal{P}(S) \backslash\{0\}$, then $S$ is infinite-dimensional.

(ii) Let $i, j, k \in\{1, \ldots, \theta\}$ be such that $q_{i i}= \pm \widetilde{q_{i j}} \in \mathbb{G}_{3}, \widetilde{q_{i k}}=1$, and also $-q_{j j}=\widetilde{q_{i j}} \widetilde{q_{j k}}=1$ or $q_{j j}^{-1}=\widetilde{q_{i j}}=\widetilde{q_{j k}} \neq-1$. If $\left[x_{i i j k}, x_{i j}\right]_{c} \in \mathcal{P}(S) \backslash\{0\}$, then $S$ is infinite-dimensional.

Proof. (i) We follow the same scheme of proof. Set

$$
y_{1}=x_{i}, \quad y_{2}=x_{j}, \quad y_{3}=\left[x_{i i j}, x_{i j}\right]_{c},
$$

and $h_{i} \in \Gamma, \eta_{i} \in \widehat{\Gamma}, i=1,2,3$ the corresponding elements. Suppose that the braiding matrix $\left(Q_{r s}=\eta_{s}\left(h_{r}\right)\right)_{1 \leq r, s \leq 3}$ appears in Heckenberger's list. The associated generalized Dynkin diagram is

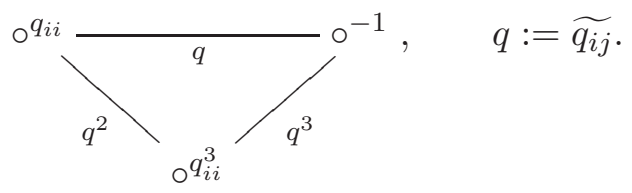

Then $Q_{33}=q_{i i}^{3} \neq 1$, so $m_{i j} \geq 3$. Moreover the diagram is connected, so it is of type super $G(3)$, the unique diagram of rank three such that some $m_{r s}$ is $\geq 3$. Therefore $1=Q_{23} Q_{32}=$ $q^{3}$, which is a contradiction, so the diagram associated to $\left(Q_{r s}\right)$ does not correspond to a finite-dimensional Nichols algebra. In consequence $S$ is infinite-dimensional.

(ii) Set $w:=\left[x_{i i j k}, x_{i j}\right]_{c}$, and denote as above $y_{1}=x_{i}, y_{2}=x_{j}, y_{3}=x_{k}, y_{4}=w$, $W$ the subspace generated by these elements, and $h_{i} \in \Gamma, \eta_{i} \in \widehat{\Gamma}, i=1,2,3,4$ the corresponding elements: suppose again that $\mathcal{B}(W)$ is a finite-dimensional Nichols algebra. Set $\zeta=q_{i i} \in \mathbb{G}_{3}$. We analyze each possible case.

- $q_{j j}=-1, \widetilde{q_{i j}} \widetilde{q_{j k}}=1$ : the diagram of $\left(Q_{r s}\right)$ becomes

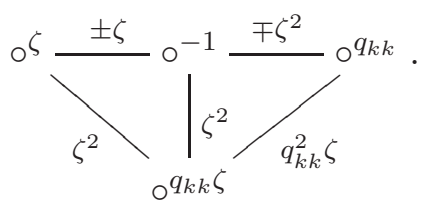

As $Q_{12} Q_{21}, Q_{14} Q_{41}, Q_{42} Q_{24} \neq 1$, and the product of these three scalars is not 1, such diagram is not in Heckenberger's list, by [H3, Lemma 9]. 
- $q_{j j}^{-1}=\widetilde{q_{i j}}=\widetilde{q_{j k}} \neq-1$ : now we have the diagram

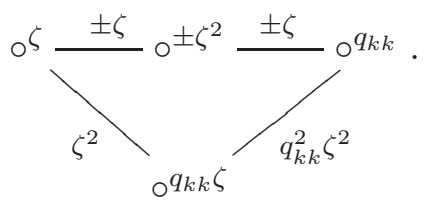

The lack of 4-cycles in Heckenberger's list implies that $1=Q_{34} Q_{43}=q_{k k}^{2} \zeta^{2}$, so $q_{k k} \zeta=-1$, because $Q_{44}=q_{k k} \zeta \neq 1$. But this diagram does not appear in [H3, Table 3].

We obtain a contradiction in all the cases, so $S$ is infinite-dimensional.

Lemma 4.4. Let $i, j, k \in\{1, \ldots, \theta\}$ be such that $\widetilde{q_{i k}}, \widetilde{q_{i j}}, \widetilde{q_{j k}} \neq 1$. Let

$$
w:=x_{i j k}-\frac{1-\widetilde{q_{j k}}}{q_{k j}\left(1-\widetilde{q_{i k}}\right)}\left[x_{i k}, x_{j}\right]_{c}-q_{i j}\left(1-\widetilde{q_{j k}}\right) x_{j} x_{i k} .
$$

If $w \in \mathcal{P}(S) \backslash\{0\}$, then $S$ is infinite-dimensional.

Proof. Set $y_{1}=x_{i}, y_{2}=x_{j}, y_{3}=x_{k}, y_{4}=w, W$ the subspace generated by these elements, $h_{i} \in \Gamma, \eta_{i} \in \widehat{\Gamma}, i=1,2,3,4$ the corresponding elements, and $\left(Q_{r s}=\eta_{s}\left(h_{r}\right)\right)_{1 \leq r, s \leq 4}$ : suppose as above that $\mathcal{B}(W)$ is finite dimensional. Note that

$$
Q_{14} Q_{41}=q_{i i}^{2} \widetilde{q_{i j}} \widetilde{q_{i k}}=q_{i i}^{2}{\widetilde{q_{j k}}}^{-1}
$$

because $\widetilde{q_{i j}} \widetilde{q_{i k}} \widetilde{q_{j k}}=1$, by [H3, Lemma 9]. By the same Lemma at least one vertex is labeled with -1 . Then, if $q_{i i}=-1$, we have that $Q_{14} Q_{41} \neq-1$; the same holds for the other vertices, so exactly one vertex is labeled with -1 (we have no 4-cycles). We look for possible braiding with these conditions in [H3, Table 3], but no one coincides with this description. Therefore $\mathcal{B}(W)$ is infinite-dimensional, and $S$ too.

Lemma 4.5. (i) Let $i, j, k \in\{1, \ldots, \theta\}$ be such that one of the following conditions holds:

- $q_{i i}=q_{j j}=-1,{\widetilde{q_{i j}}}^{2}=\widetilde{q_{j k}}-1, \widetilde{q_{i k}}=1$, or

- $\widetilde{q_{i j}}=q_{j j}=-1, q_{i i}=-{\widetilde{q_{j k}}}^{2} \in \mathbb{G}_{3}, \widetilde{q_{i k}}=1$, or

- $q_{k k}=\widetilde{q_{j k}}=q_{j j}=-1, q_{i i}=-\widetilde{q_{i j}} \in \mathbb{G}_{3}, \widetilde{q_{i k}}=1$, or

- $q_{j j}=-1, \widetilde{q_{i j}}=q_{i i}^{-2}, q_{k k}={\widetilde{q_{j k}}}^{-1}=-q_{i i}^{3}, \widetilde{q_{i k}}=1$, or

- $q_{i i}=q_{j j}=q_{k k}=-1, \pm \widetilde{q_{i j}}=\widetilde{q_{j k}} \in \mathbb{G}_{3}, \widetilde{q_{i k}}=1$,

If $\left[\left[x_{i j}, x_{i j k}\right]_{c}, x_{j}\right]_{c} \in \mathcal{P}(S) \backslash\{0\}$, then $S$ is infinite-dimensional.

(ii) Let $i, j, k \in\{1, \ldots, \theta\}$ be such that $q_{i i}=q_{j j}=-1,\left(\widetilde{q_{i j}}\right)^{3}=\left(\widetilde{q_{j k}}\right)^{-1}, \widetilde{q_{i k}}=1$. If $\left[\left[x_{i j},\left[x_{i j}, x_{i j k}\right]_{c}\right]_{c}, x_{j}\right]_{c} \in \mathcal{P}(S) \backslash\{0\}$, then $S$ is infinite-dimensional.

Proof. (i) Set $y_{1}=x_{i}, y_{2}=x_{j}, y_{3}=x_{k}, y_{4}=\left[\left[x_{i j}, x_{i j k}\right]_{c}, x_{j}\right]_{c}, W$ the subspace generated by these elements, $h_{i} \in \Gamma, \eta_{i} \in \widehat{\Gamma}, i=1,2,3,4$ the associated elements and $\left(Q_{r s}=\right.$ $\left.\eta_{s}\left(h_{r}\right)\right)_{1 \leq r, s \leq 4}$ the braiding matrix. We will consider the associated generalized Dynkin diagram for each case.

For the first case, we have the following diagram, where $q:=\widetilde{q_{i j}}$ :

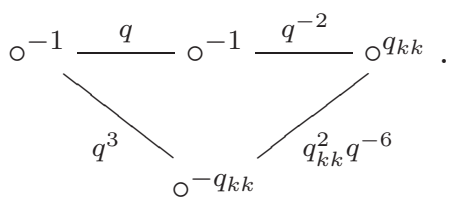


Suppose that $\mathcal{B}(W)$ is finite-dimensional. Then $Q_{33} Q_{32} Q_{23}=1$, so $q_{k k}=q^{2}$ and then $Q_{34} Q_{43}=q^{-2} \neq 1$. In consequence such diagram is of type super $F(4)$. Then $1=$ $Q_{14} Q_{41}=q^{3}$, which is a contradiction.

For the second case, $Q_{12} Q_{21}=q_{i i} \in \mathbb{G}_{3}$, and $Q_{14} Q_{41}=-1$, so the diagram contains a 4-cycle and then $\mathcal{B}(W)$ is infinite-dimensional. An analogous situation holds for the third case, because $Q_{12} Q_{21}=-q_{i i} \in \mathbb{G}_{6}$, and $Q_{14} Q_{41}=-1$.

For the fourth case, $\widetilde{Q_{23}}=\widetilde{Q_{24}}=\widetilde{Q_{34}}=\widetilde{q_{k j}} \neq 1$ and $\widetilde{Q_{14}}=\widetilde{Q_{12}}=\widetilde{q_{i j}} \neq 1$, so $\mathcal{B}(W)$ is infinite-dimensional.

For the last case, $Q_{44}=1$, and then $\mathcal{B}(W)$ is infinite-dimensional.

Therefore $S$ is infinite-dimensional in all the cases.

(ii) We use the same notation, but in this case $y_{4}=\left[\left[x_{i j},\left[x_{i j}, x_{i j k}\right]_{c}\right]_{c}, x_{j}\right]_{c}$. So we have the following diagram for $\left(Q_{r s}\right)$ :

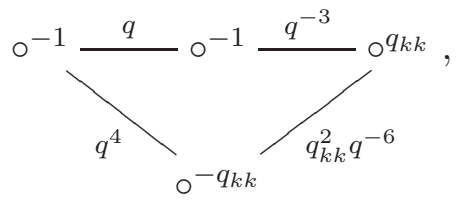

where $q=\widetilde{q_{i j}}$. Suppose that $\mathcal{B}(W)$ is finite-dimensional. By [H3, Table 3], this diagram cannot be connected. In consequence, $1=Q_{41} Q_{14}=Q_{34} Q_{43}$, so $q_{k k}= \pm 1$. But then $Q_{33}=1$, or $Q_{44}=1$, which is a contradiction to the fact that $\mathcal{B}(W)$ is finite-dimensional. So $S$ is infinite-dimensional.

Lemma 4.6. Let $i, j, k, l \in\{1, \ldots, \theta\}$ be such that $q_{j j} \widetilde{q_{i j}}=q_{j j} \widetilde{q_{j k}}=1,{\widetilde{q_{j k}}}^{2}={\widetilde{q_{k l}}}^{-1}=q_{l l}$, $q_{k k}=-1, \widetilde{q_{i k}}=\widetilde{q_{i l}}=\widetilde{q_{j l}}$. If $\left[\left[\left[x_{i j k l}, x_{k}\right]_{c}, x_{j}\right]_{c}, x_{k}\right]_{c} \in \mathcal{P}(S) \backslash\{0\}$, then $S$ is infinitedimensional.

Proof. We use a similar notation and consider the corresponding subspace $W$ generated by the corresponding primitive elements. Suppose that $\mathcal{B}(W)$ is finite-dimensional. Its associated Dynkin diagram is

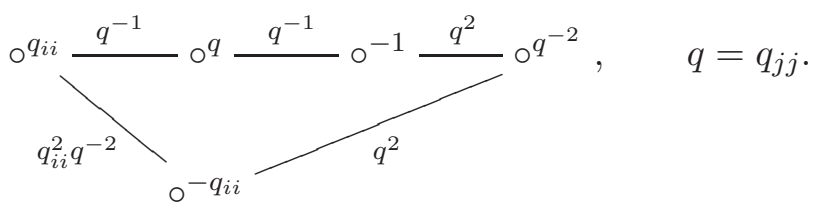

Note that $1=Q_{15} Q_{51}$, because there are no 5 -cycles and $q^{2} \neq 1$. Therefore $q_{i i}=q$, but this diagram is not in Heckenberger's list, so $\mathcal{B}(W)$ is infinite-dimensional, and $S$ too.

Lemma 4.7. Let $i, j, k \in\{1, \ldots, \theta\}$ be such that $q_{j j}={\widetilde{q_{i j}}}^{-1}=\widetilde{q_{j k}}$.

(i) If $q_{j j} \in \mathbb{G}_{3}$ and $\left[\left[x_{i j k}, x_{j}\right]_{c} x_{j}\right]_{c} \in \mathcal{P}(S) \backslash\{0\}$, then $S$ is infinite-dimensional.

(ii) If $q_{j j} \in \mathbb{G}_{4}$ and $\left[\left[\left[x_{i j k}, x_{j}\right]_{c}, x_{j}\right]_{c}, x_{j}\right]_{c} \in \mathcal{P}(S) \backslash\{0\}$, then $S$ is infinite-dimensional.

Proof. (i) Using the same notation as in previous Lemmata, we have the diagram

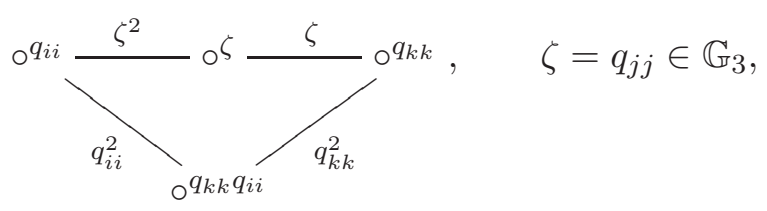

for $y_{1}=x_{i}, y_{2}=x_{j}, y_{3}=x_{k}, y_{4}=\left[\left[x_{i j k}, x_{j}\right]_{c} x_{j}\right]_{c}$, with corresponding matrix $\left(Q_{r s}\right)$, and $W$ is the subspace generated by these elements. Note that 
- if $q_{i i}=q_{k k}=-1$, then $Q_{44}=1$;

- if $q_{i i}, q_{k k} \neq-1$, then the diagram contains a 4-cycle;

- if $q_{i i}=-1, q_{k k} \neq-1$, or $q_{i i} \neq-1, q_{k k}=-1$, the diagram contains $\circ^{q} \frac{q^{2}}{\circ^{-q}}$ as a subdiagram (where $q=q_{i i}$ or $q=q_{k k}$ ), and this connected subdiagram of rank two is not in [H3, Table 1].

In all the cases $\mathcal{B}(W)$ is infinite-dimensional, so $S$ too.

(ii) The proof is analogous.

Lemma 4.8. (i) Let $i, j, k, l \in\{1, \ldots, \theta\}$ be such that $q_{l l}={\widetilde{q_{l k}}}^{-1}=q_{k k}={\widetilde{q_{j k}}}^{-1}=q^{2}$, $\widetilde{q_{i j}}=q_{i i}^{-1}=q^{3}$ for some $q \in \mathbf{k}^{\times}, q_{j j}=-1, \widetilde{q_{i k}}=\widetilde{q_{i l}}=\widetilde{q_{j l}}=1$. If $\left[\left[\left[x_{i j k}, x_{j}\right]_{c},\left[x_{i j k l}, x_{j}\right]_{c}\right]_{c}, x_{j k}\right]_{c} \in \mathcal{P}(S) \backslash\{0\}$, then $S$ is infinite-dimensional.

(ii) Let $i, j, k, l \in\{1, \ldots, \theta\}$ be such that $\widetilde{q_{j k}}=\widetilde{q_{i j}}=q_{j j}^{-1} \in \mathbb{G}_{4}^{\prime} \cup \mathbb{G}_{6}^{\prime}, q_{i i}=q_{k k}=-1$, $\widetilde{q_{i k}}=\widetilde{q_{i l}}=\widetilde{q_{j l}}=1, \widetilde{q_{j k}}=\widetilde{q_{l k}}$. If $\left[\left[x_{i j k},\left[x_{i j k l}, x_{k}\right]_{c}\right]_{c}, x_{j k}\right]_{c} \in \mathcal{P}(S) \backslash\{0\}$, then $S$ is infinite-dimensional.

Proof. We use the same notation and strategy as in the previous Lemmata.

(i) We have that $\widetilde{Q_{15}}=q^{3} \neq 1$, and the diagram corresponding to $\left(q_{s t}\right)$ does not admit extensions with finite root systems. Therefore $S$ is infinite-dimensional.

(ii) Now, $\widetilde{Q_{15}}=q^{3} \neq 1$, and we conclude the same as in (i).

Lemma 4.9. Let $i, j, k, l \in\{1, \ldots, \theta\}$ be such that one of the following situations hold:

$$
\begin{aligned}
& \circ q_{k k}=-1, q_{i i}=\widetilde{q_{i j}}-1=q_{j j}^{2}, \widetilde{q_{k l}}=q_{l l}^{-1}=q_{j j}^{3}, \widetilde{q_{j k}}=q_{j j}^{-1}, \widetilde{q_{i k}}=\widetilde{q_{i l}}=\widetilde{q_{j l}}=1, \\
& \circ q_{i i}=\widetilde{q_{i j}}-1=-q_{l l}^{-1}=-\widetilde{q_{k l}}, q_{j j}=\widetilde{q_{j k}}=q_{k k}=-1, \widetilde{q_{i k}}=\widetilde{q_{i l}}=\widetilde{q_{j l}}=1, \text { or } \\
& \circ q_{j j}=\widetilde{q_{j k}}-1 \in \mathbb{G}_{3}, q_{i i}=\widetilde{q_{i j}}-1=q_{l l}=\widetilde{q_{k l}}-1=-q_{j j}, q_{k k}=-1, \widetilde{q_{i k}}=\widetilde{q_{i l}}=\widetilde{q_{j l}}=1 .
\end{aligned}
$$

If $\left[\left[x_{i j k l}, x_{j}\right]_{c}, x_{k}\right]_{c}-q_{j k}\left({\widetilde{q_{i j}}}^{-1}-q_{j j}\right)\left[\left[x_{i j k l}, x_{k}\right]_{c}, x_{j}\right]_{c} \in \mathcal{P}(S) \backslash\{0\}$, then $S$ is infinitedimensional.

Proof. Using the same notation and strategy as in the previous Lemmata, we compute the diagrams for $\left(Q_{r s}\right)$ in each case, and note that the diagrams of $\left(q_{r s}\right)$ does not admit extensions with finite root systems.

- In the first case, $\widetilde{Q_{35}}=q_{j j} \neq 1$.

- In the second case, $\widetilde{Q_{25}}=\widetilde{q_{i j}} \neq 1$.

- For the last one, $\widetilde{Q_{35}}=-q_{j j}^{2} \neq 1$.

Therefore $S$ is infinite-dimensional in any case.

Lemma 4.10. (i) Let $i, j, k \in\{1, \ldots, \theta\}$ be such that $q_{k k}=q_{j j}={\widetilde{q_{i j}}}^{-1}={\widetilde{q_{j k}}}^{-1} \in \mathbb{G}_{9}$, $\widetilde{q_{i k}}=1, q_{i i}=q_{k k}^{6}$. If $\left[\left[x_{i i j}, x_{i i j k}\right]_{c}, x_{i j}\right]_{c} \in \mathcal{P}(S) \backslash\{0\}$, then $S$ is infinite-dimensional.

(ii) Let $i, j, k \in\{1, \ldots, \theta\}$ be such that $q_{i i}={\widetilde{q_{i j}}}^{-1} \in \mathbb{G}_{9}, q_{j j}={\widetilde{q_{j k}}}^{-1}=q_{i i}^{5}, \widetilde{q_{i k}}=1$, $q_{k k}=q_{i i}^{6}$. If $\left[\left[x_{i j k}, x_{j}\right]_{c}, x_{k}\right]_{c}-\left(1+\widetilde{q_{j k}}\right)^{-1} q_{j k}\left[\left[x_{i j k}, x_{k}\right]_{c}, x_{j}\right]_{c} \in \mathcal{P}(S) \backslash\{0\}$, then $S$ is infinite-dimensional.

Proof. We use the same notation and strategy as in the previous Lemmata, and note also that these diagrams does not admit extensions with finite root systems.

(i) In this case, $\widetilde{Q_{34}}=\widetilde{q_{j k}} \neq-1$, so $S$ is infinite-dimensional, because the diagram of $\left(Q_{r s}\right)$ is connected and contains the diagram of $\left(q_{i j}\right)$.

(ii) Now, $\widetilde{Q_{34}}=q_{j j} \neq-1$, and $S$ is also infinite-dimensional. 
Lemma 4.11. (i) Let $i, j, k \in\{1, \ldots, \theta\}$ be such that $q_{i i}=q_{k k}=\widetilde{q_{i j}}=-1, q_{j j}=\widetilde{q_{j k}}-1$, $\widetilde{q_{i k}}=1$. If $\left[x_{i j}, x_{i j k}\right]_{c} \in \mathcal{P}(S) \backslash\{0\}$, then $S$ is infinite-dimensional.

(ii) Let $i, j, k \in\{1, \ldots, \theta\}$ be such that $q_{i i}=q_{k k}=-1, \widetilde{q_{i j}} \in \mathbb{G}_{3}, q_{j j}= \pm \widetilde{q_{i j}}=-\widetilde{q_{j k}}$, $\widetilde{q_{i k}}=1$. If $\left[x_{i}, x_{j j k}\right]_{c}-\left(1+q_{j j}^{2}\right) q_{k j}^{-1}\left[x_{i j k}, x_{j}\right]_{c}-\left(1+q_{j j}\right)\left(1+q_{j j}^{2}\right) q_{i j} x_{j} x_{i j k} \in \mathcal{P}(S) \backslash\{0\}$, then $S$ is infinite-dimensional.

(iii) Let $i, j, k \in\{1, \ldots, \theta\}$ be such that $\widetilde{q_{j k}}=1, q_{i i}=\widetilde{q_{i j}}=-\widetilde{q_{i k}} \in \mathbb{G}_{3}$. If $\left[x_{i},\left[x_{i j}, x_{i k}\right]_{c}\right]_{c}+$ $q_{j k} q_{i k} q_{j i}\left[x_{i i k}, x_{i j}\right]_{c}+q_{i j} x_{i j} x_{i i k} \in \mathcal{P}(S) \backslash\{0\}$, then $S$ is infinite-dimensional.

(iv) Let $i, j, k \in\{1, \ldots, \theta\}$ be such that $q_{j j}=q_{k k}=\widetilde{q_{j k}}=-1, q_{i i}=-\widetilde{q_{i j}} \in \mathbb{G}_{3}, \widetilde{q_{i k}}=1$. If $\left[x_{i i j k}, x_{i j k}\right]_{c} \in \mathcal{P}(S) \backslash\{0\}$, then $S$ is infinite-dimensional.

Proof. We consider the same notation as before. We consider the subspace $W$ generated by $y_{1}=x_{i}, y_{2}=x_{j}, y_{3}=x_{k}$ and $y_{4}$ (the primitive element corresponding to the relation), where $y_{i} \in W_{h_{i}}^{\eta_{i}}$, for some $h_{i} \in \Gamma, \eta_{i} \in \widehat{\Gamma}$, and set $\left(Q_{r s}=\eta_{s}\left(h_{r}\right)\right)$. We will prove again that $\mathcal{B}(W)$ is infinite-dimensional.

(i) The corresponding diagram of $\left(Q_{r s}\right)$ is

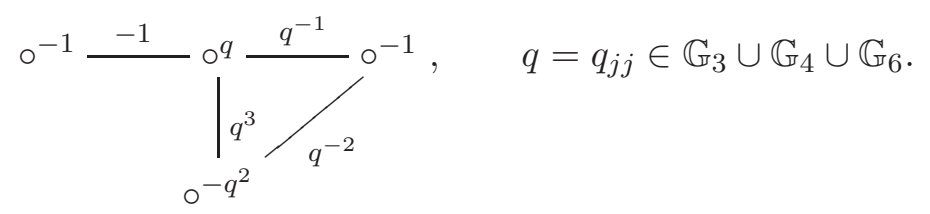

If $q \in \mathbb{G}_{4}$ then $Q_{44}=1$, so $\mathcal{B}(W)$ is infinite-dimensional. If $q \in \mathbb{G}_{3} \cup \mathbb{G}_{6}$, the diagram is not in [H3, Table 3], so $\mathcal{B}(W)$ is also again infinite-dimensional.

(ii) We note that $Q_{14} Q_{41}=Q_{34} Q_{43}=q_{j j}^{2} \in \mathbb{G}_{3}$. Therefore, the diagram corresponding to $\left(Q_{r s}\right)$ contains a 4-cycle, and then $\mathcal{B}(W)$ is infinite-dimensional.

(iii) In this case, $Q_{24} Q_{42}=-q_{i i}^{2}$ and $Q_{34} Q_{43}=-q_{k k}^{2} \neq 1$, because $q_{k k} \in\left\{-1, \widetilde{q_{i k}}-1\right\}$. The diagram corresponding to $\left(Q_{r s}\right)_{r, s=2,3,4}$ is a 3-cycle such that $\widetilde{Q_{24}} \widetilde{Q_{34}} \widetilde{Q_{23}} \neq 1$. [H3, Lemma 9] implies that $\mathcal{B}(W)$ is infinite-dimensional.

(iv) $\mathcal{B}(W)$ is infinite-dimensional because $Q_{44}=1$.

Lemma 4.12. Let $i, j \in\{1, \ldots, \theta\}$ be such that the satisfy one of the following conditions: (i) $-q_{i i},-q_{j j}, q_{i i} \widetilde{q_{i j}}, q_{j j} \widetilde{q_{i j}} \neq 1$,

$$
\left[x_{i},\left[x_{i j}, x_{j}\right]_{c}\right]_{c}-\frac{\left(1+q_{j j}\right)\left(1-q_{j j} \widetilde{q_{i j}}\right)}{\left(1-\widetilde{q_{i j}}\right) q_{j j} q_{j i}} x_{i j}^{2} \in \mathcal{P}(S) \backslash\{0\}
$$

(ii) $q_{j j}=-1, q_{i i} \widetilde{q_{i j}} \notin \mathbb{G}_{6}$, and also $m_{i j} \in\{4,5\}$, or $m_{i j}=3, q_{i i} \in \mathbb{G}_{4}$,

$$
\left[x_{i}, x_{3 \alpha_{i}+2 \alpha_{j}}\right]_{c}-\frac{1-q_{i i} \widetilde{q_{i j}}-q_{i i}^{2}{\widetilde{q_{i j}}}^{2} q_{j j}}{\left(1-q_{i i} \widetilde{q_{i j}}\right) q_{j i}} x_{i i j}^{2} \in \mathcal{P}(S) \backslash\{0\} ;
$$

(iii) $4 \alpha_{i}+3 \alpha_{j} \notin \Delta_{+}^{\chi}, q_{j j}=-1$ or $m_{j i}=2$, and also $m_{i j} \geq 3$ or $m_{i j}=2, q_{i i} \in \mathbb{G}_{3}$,

$$
\left[x_{3 \alpha_{i}+2 \alpha_{j}}, x_{i j}\right]_{c} \in \mathcal{P}(S) \backslash\{0\} ;
$$

(iv) $3 \alpha_{i}+2 \alpha_{j} \in \Delta_{+}^{\chi}, 5 \alpha_{i}+3 \alpha_{j} \notin \Delta_{+}^{\chi}$, and $q_{i i}^{3} \widetilde{q_{i j}}, q_{i i}^{4} \widetilde{q_{i j}} \neq 1,\left[x_{i i j}, x_{3 \alpha_{i}+2 \alpha_{j}}\right]_{c} \in \mathcal{P}(S) \backslash\{0\}$;

(v) $4 \alpha_{i}+3 \alpha_{j} \in \Delta_{+}^{\chi}, 5 \alpha_{i}+4 \alpha_{j} \notin \Delta_{+}^{\chi},\left[x_{4 \alpha_{i}+3 \alpha_{j}}, x_{i j}\right]_{c} \in \mathcal{P}(S) \backslash\{0\}$;

(vi) $5 \alpha_{i}+2 \alpha_{j} \in \Delta_{+}^{\chi}, 7 \alpha_{i}+3 \alpha_{j} \notin \Delta_{+}^{\chi}$, [[ $\left.\left.x_{i i i j}, x_{i i j}\right], x_{i i j}\right]_{c} \in \mathcal{P}(S) \backslash\{0\}$;

(vii) $q_{j j}=-1,5 \alpha_{i}+4 \alpha_{j} \in \Delta_{+}^{\chi},\left[x_{i i j}, x_{4 \alpha_{i}+3 \alpha_{j}}\right]_{c}-a x_{3 \alpha_{i}+2 \alpha_{j}}^{2} \in \mathcal{P}(S) \backslash\{0\}$, for some $a \in \mathbf{k}^{\times}$.

Then $S$ is infinite-dimensional. 
Proof. Firstly we note that there exists just one connected generalized Dynkin diagram of rank three such that $3 \alpha_{i}+2 \alpha_{j} \in \Delta_{+}^{\chi}$, for some pair $i, j$, which is exactly the unique one such that $m_{k l} \geq 3$ for some pair $k$, l. Moreover, $4 \alpha_{i}+3 \alpha_{j}, 5 \alpha_{i}+4 \alpha_{j}$, are not positive roots for any pair $i, j$ and any connected Dynkin diagram of rank 3.

We consider as above the subspace $W$ generated by $y_{1}=x_{i}, y_{2}=x_{j}$ and $y_{3}$, the relation which is a primitive element by hypothesis, and analyze its generalized Dynkin diagram.

(i) If $Q_{13} Q_{31} \neq 1$ or $Q_{23} Q_{32} \neq 1$, then $\mathcal{B}(W)$ is infinite-dimensional. In other case,

$$
Q_{13} Q_{31}=q_{i i}^{4} q_{i j}^{2} q_{j i}^{2}=1, \quad Q_{23} Q_{32}=q_{j j}^{4} q_{i j}^{2} q_{j i}^{2}=1,
$$

so $Q_{33}=q_{i i}^{4} q_{i j}^{4} q_{j i}^{4} q_{j j}^{4}=1$, and $\mathcal{B}(W)$ is also infinite-dimensional.

(ii) If $q_{i i} \in \mathbb{G}_{4}, \widetilde{q_{i j}}=q_{i i}=q_{j j}^{-1}$ (and then $\left(q_{r s}\right)$ is Cartan of type $G_{2}$ ), then

$$
Q_{33}=q_{i i}^{16} q_{i j}^{8} q_{j i}^{8} q_{j j}^{4}=1,
$$

so $\mathcal{B}(W)$ is infinite-dimensional. In other case, $Q_{13} Q_{31} \neq 1$, or $Q_{23} Q_{32} \neq 1$, or

$$
Q_{13} Q_{31}=q_{i i}^{8} q_{i j}^{2} q_{j i}^{2}=1, Q_{23} Q_{32}=q_{i j}^{4} q_{j i}^{4} q_{j j}^{4}, \quad \text { so } Q_{33}=1,
$$

and therefore $\mathcal{B}(W)$ is infinite-dimensional.

(iii) Now we calculate

$$
Q_{33}=q_{i i}^{16} q_{i j}^{12} q_{j i}^{12} q_{j j}^{9}, \quad Q_{13} Q_{31}=q_{i i}^{8} q_{i j}^{3} q_{j i}^{3}, \quad Q_{23} Q_{32}=q_{i j}^{4} q_{j i}^{4} q_{j j}^{6} .
$$

If $\left(q_{r s}\right)$ is Cartan of type $G_{2}$ and $q_{i i} \in \mathbb{G}_{6}, \widetilde{q_{i j}}=q_{j j}=-1$, then $\mathcal{B}(W)$ is infinitedimensional, because we have a connected diagram of rank three such that $M_{12}=3$, and it is not of type super $G(3)$. In other case, we will prove that $Q_{13} Q_{31} \neq 1$ or $Q_{23} Q_{32} \neq 1$ to conclude that $\mathcal{B}(W)$ is infinite-dimensional. If $m_{j i} \geq 2$, we have the following possible cases:

- $q_{i i}=-\zeta, \widetilde{q_{i j}}=\zeta^{7}, q_{j j}=\zeta^{3}, \zeta \in \mathbb{G}_{9} ;$ in such case, $Q_{23} Q_{32}=\zeta$.

- $q_{i i}=-\zeta, \widetilde{q_{i j}}=-\zeta^{12}, q_{j j}=\zeta^{5}, \zeta \in \mathbb{G}_{15}$; therefore, $Q_{23} Q_{32}=\zeta^{3}$.

Also, if $q_{i i}=\zeta^{8}, \widetilde{q_{i j}}=\zeta^{3}, q_{j j}=-1, \zeta \in \mathbb{G}_{12}$, then $Q_{13} Q_{31}=\zeta$. In all the remaining cases, $q_{j j}=-1$ and $\widetilde{q_{i j}} \notin \mathbb{G}_{4}$, so $Q_{23} Q_{32} \neq 1$.

(iv) This relation is not redundant just in the following two cases:

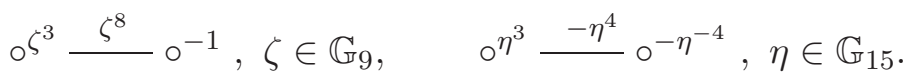

Note that they are not contained in any connected diagram of rank three in [H3, Table $2]$, so it is enough to verify that $Q_{13} Q_{31} \neq 1$ or $Q_{23} Q_{32} \neq 1$ to conclude that $\mathcal{B}(W)$ is infinite-dimensional. For the first diagram, $Q_{23} Q_{32}=\zeta^{4} \neq 1$; and for the second one, $Q_{23} Q_{32}=-\eta^{-4} \neq 1$.

(v) The proof is analogous to (iii). Note that

$$
Q_{33}=q_{i i}^{25} q_{i j}^{12} q_{j i}^{20} q_{j j}^{16}, \quad Q_{13} Q_{31}=q_{i i}^{10} q_{i j}^{4} q_{j i}^{4}, \quad Q_{23} Q_{32}=q_{i j}^{5} q_{j i}^{5} q_{j j}^{8} .
$$

We have that $q_{j j}=-1$ for every diagram satisfying the conditions for this item. Also, if $q_{i i}=\zeta \in \mathbb{G}_{5}, \widetilde{q_{i j}}=\zeta^{2}$, it follows that $Q_{33}=1$. In the remaining cases, $\widetilde{q_{i j}} \notin \mathbb{G}_{5}$, so $Q_{32} Q_{23} \neq-1$, and then $\mathcal{B}(W)$ is infinite-dimensional.

(vi) In this case,

$$
Q_{33}=q_{i i}^{49} q_{i j}^{21} q_{j i}^{21} q_{j j}^{9}, \quad Q_{13} Q_{31}=q_{i i}^{14} q_{i j}^{3} q_{j i}^{3}, \quad Q_{23} Q_{32}=q_{i j}^{7} q_{j i}^{7} q_{j j}^{6} .
$$

We have that $q_{j j}=-1$ for every diagram satisfying the conditions for this item, and also $\widetilde{q_{i j}} \notin \mathbb{G}_{7}$, so $Q_{23} Q_{32} \neq 1$. Therefore $\mathcal{B}(W)$ is infinite-dimensional.

(vii) The proof is analogous to the one for (i) . 
We conclude that $S$ is infinite-dimensional in all the cases.

Now we can prove the main Theorem of this Section.

Theorem 4.13. Let $S=\oplus_{n \geq 0} S(n)$ be a finite-dimensional graded Hopf algebra in ${ }_{\mathrm{k} \Gamma}^{\mathrm{k}} \mathcal{Y} \mathcal{D}$, where $\Gamma$ is a finite abelian group, such that $S(0)=\mathbf{k} 1$. If $S$ is generated as an algebra by $S(0) \oplus S(1)$, then $S \cong \mathcal{B}(V)$.

Proof. Fix a basis $x_{1}, \ldots, x_{\theta}$ of $V:=S(1)$, such that $x_{i} \in S(1)_{g_{i}}^{\chi_{i}}$ for some $g_{i} \in \Gamma$ and $\chi_{i} \in \widehat{\Gamma}$, and set $q_{i j}:=\chi_{j}\left(g_{i}\right)$.

As $S$ is generated as an algebra by $S(0) \oplus S(1)$, the canonical projection $T(V) \rightarrow$ $\mathcal{B}(V)=T(V) / I(V)$ induces a surjective morphism $\pi: S \rightarrow \mathcal{B}(V)$ of graded braided Hopf algebras; we can consider $S=T(V) / I$, for some graded braided Hopf ideal $I$ of $T(V)$, generated by homogeneous elements of degree $\geq 2, I \subseteq I(V)$.

Suppose that $I(V) \supsetneqq I$. Then at least one of the generators of $I(V)$ from Theorem 3.1 does not belong to $I$. We can assume that $\mathrm{x} \in I(V) \backslash I$ is one of these generators, of minimal degree $k$. Then $\mathrm{x}$ is primitive in $S$ by Lemma 3.2

By Proposition 4.1 and Lemmata 4.2,4.12, we deduce that $\mathbf{x}=x_{\alpha}^{N_{\alpha}}$ for some $\alpha \in \mathcal{O}$, or a simple root $\alpha=\alpha_{i}$ such that $i$ is not a Cartan vertex, or $\alpha=\alpha_{i}+\alpha_{j}$, such that $N_{\alpha}=2, q_{i i}=q_{j j}=\widetilde{q_{i j}}=-1$. If $g_{\alpha} \in \Gamma, \chi_{\alpha} \in \widehat{\Gamma}$ are the associated elements, we have that $q_{\alpha}=\chi_{\alpha}\left(g_{\alpha}\right)$, which is a root of unity of order $N_{\alpha}$. Therefore $g_{\alpha}^{N_{\alpha}} \in \Gamma$ and $\chi_{\alpha}^{N_{\alpha}} \in \widehat{\Gamma}$ are the associated elements to $\mathbf{x}$, and

$$
c(\mathbf{x} \otimes \mathbf{x})=g_{\alpha}^{N_{\alpha}} \cdot \mathbf{x} \otimes \mathbf{x}=\chi_{\alpha}^{N_{\alpha}}\left(g_{\alpha}^{N_{\alpha}}\right) \mathbf{x} \otimes \mathbf{x}=\mathbf{x} \otimes \mathbf{x},
$$

so $\mathbf{x}$ generates in $S$ an infinite-dimensional braided Hopf subalgebra, and we obtain a contradiction. In consequence, $S \cong \mathcal{B}(V)$.

Remark 4.14. Note that we just use the fact that the braiding is diagonal, so we can generalize this Theorem to a general braided Hopf algebra $R$ in ${ }_{H}^{H} \mathcal{Y} \mathcal{D}$, where $H$ is a finite-dimensional Hopf algebra which acts diagonally over $R(1)$.

The following Theorem answers positively Conjecture 1 in the case that the group of group-like elements is abelian. It extends [AS4, Thm. 5.5].

Theorem 4.15. Let $H$ be a finite-dimensional pointed Hopf algebra over an abelian group $\Gamma$. Then $H$ is generated by its group-like and skew-primitive elements.

Proof. Let gr $H=R \# \mathbf{k} \Gamma, V=R(1)$. Then $H$ is generated by its group-like and skewprimitive elements if and only if gr $H$ satisfies this condition, which is equivalent to the fact that $R$ is the Nichols algebra $\mathcal{B}(V)$. Let $S$ be the graded dual $R^{*}$ in the category ${ }_{G}^{G} \mathcal{Y D}$, which is generated as an algebra by $S(1)=V^{*}$. By AS3, Lemma 2.3] it is enough to prove that $S$ is the Nichols algebra $\mathcal{B}\left(V^{*}\right)$, which follows by Theorem 4.13 ,

\section{REFERENCES}

[An] N. Andruskiewitsch, About finite dimensional Hopf algebras, Notes of a course given at the CIMPA School Quantum symmetries in theoretical physics and mathematics Bariloche 2000. Contemp. Math. 294, 1-57 (2002).

[AA] N. Andruskiewitsch and I. Angiono, On Nichols algebras with generic braiding, Modules and Comodules. Trends in Mathematics. Brzezinski, T.; Gómez Pardo, J.L.; Shestakov, I.; Smith, P.F. (Eds.), pp. 47-64 (2008). ISBN: 978-3-7643-8741-9.

[AAY] N. Andruskiewitsch, I. Angiono and H. Yamane, On pointed Hopf superalgebras. Contemp. Math. 544 (2011), 123-140.

[AS1] N. Andruskiewitsch and H.-J. Schneider, Lifting of quantum linear spaces and pointed Hopf algebras of order $p^{3}$, J. Algebra 209, 658-691 (1998). 
[AS2] N. Andruskiewitsch and H.-J. Schneider, Finite quantum groups and Cartan matrices, Adv. Math. 154, 1-45 (2000).

[AS3] N. Andruskiewitsch and H.-J. Schneider, Pointed Hopf algebras, "New directions in Hopf algebras", MSRI series Cambridge Univ. Press; 1-68 (2002).

[AS4] N. Andruskiewitsch and H.-J. Schneider, On the classification of finite-dimensional pointed Hopf algebras, Annals of Mathematics Vol. 171 (2010), No. 1, 375417.

[A1] I. Angiono, On Nichols algebras with standard braiding, Algebra and Number Theory Vol. 3, No. 1, 35-106, (2009).

[A2] I. Angiono, A presentation by generators and relations of Nichols algebras of diagonal type and convex orders on root systems, submitted. arXiv:1008.4144.

[AGI] I. Angiono and A. García Iglesias. Pointed Hopf algebras with standard braiding are generated in degree one, Contemp. Math. 537 (2011), 57-70.

[CH1] M. Cuntz and I. Heckenberger, Weyl groupoids with at most three objects. J. Pure Appl. Algebra 213, No. 6 (2009), 1112-1128.

[CH2] M. Cuntz and I. Heckenberger, Finite Weyl groupoids of rank three, to appear in Trans. Amer. Math. Soc. (2010). math. QA/0912.0212.

[GHV] M. Graña, I. Heckenberger and L. Vendramín, SARNA: Arithmetic Root Systems and Nichols Algebras Software, a program to calculate the Arithmetic Root System associated to a braid of diagonal type. Available at http://mate.dm.uba.ar/ lvendram/

[H1] I. Heckenberger, Weyl equivalence for rank 2 Nichols algebras of diagonal type. Ann. Univ. Ferrara - Sez. VII - Sc. Mat., 281-289 (2005).

[H2] I. Heckenberger, The Weyl groupoid of a Nichols algebra of diagonal type, Invent. Math. 164, 175-188 (2006).

[H3] I. Heckenberger, Classification of arithmetic root systems, Adv. Math. 220 (2009) 59-124.

[H4] I. Heckenberger, Lusztig isomorphisms for Drinfel'd doubles of bosonizations of Nichols algebras of diagonal type, J. Alg. 323 (2010), 2130-2180.

[HS] I. Heckenberger and H.-J. Schneider, Right coideal subalgebras of Nichols algebras and the Duflo order on the Weyl groupoid. math.QA/0909.0293.

[HY] I. Heckenberger and H. Yamane, A generalization of Coxeter groups, root systems, and Matsumoto's theorem, Math. Z. 259 (2008), 255-276.

[He] M. Helbig, On the lifting of Nichols algebras, math. QA/1003.5882.

[Kh] V. Kharchenko, A quantum analog of the Poincare-Birkhoff-Witt theorem, Algebra and Logic 38, (1999), 259-276.

[L] G. Lusztig, Introduction to quantum groups, Birkhäuser (1993).

[Mo] S. Montgomery, Hopf algebras and their action on rings, CBMS Regional Conference Series 82 (1993).

[R] M. Rosso, Lyndon words and Universal R-matrices, talk at MSRI, October 26, 1999, available at http://www.msri.org, Lyndon basis and the multiplicative formula for $R$-matrices, preprint (2003).

[Se] V. Serganova, On generalizations of root systems. Commun. Algebra 24 (1996), 4281-4299.

[U] S. Ufer, PBW bases for a class of braided Hopf algebras, J. Alg. 280 (2004) 84-119.

[Y] H. Yamane, Quantized enveloping algebras associated to simple Lie superalgebras and their universal R-matrices Publ. Res. Inst. Math. Sci. 30 (1994), 15-87.

Famaf-Ciem (COnicet), Universidad Nacional de Córdoba, Medina Allende s/n, Ciudad Universitaria, 5000 Córdoba, República Argentina.

E-mail address: angiono@famaf.unc.edu.ar 